\title{
The Impact of Co-presence and Visual Elements in 3D VLEs on Interpersonal Emotional Connection in Telecollaboration
}

Hisae Matsui

West Virginia University

Follow this and additional works at: https://researchrepository.wvu.edu/etd

\section{Recommended Citation}

Matsui, Hisae, "The Impact of Co-presence and Visual Elements in 3D VLEs on Interpersonal Emotional Connection in Telecollaboration" (2014). Graduate Theses, Dissertations, and Problem Reports. 200. https://researchrepository.wvu.edu/etd/200

This Dissertation is protected by copyright and/or related rights. It has been brought to you by the The Research Repository @ WVU with permission from the rights-holder(s). You are free to use this Dissertation in any way that is permitted by the copyright and related rights legislation that applies to your use. For other uses you must obtain permission from the rights-holder(s) directly, unless additional rights are indicated by a Creative Commons license in the record and/ or on the work itself. This Dissertation has been accepted for inclusion in WVU Graduate Theses, Dissertations, and Problem Reports collection by an authorized administrator of The Research Repository @ WVU.

For more information, please contact researchrepository@mail.wvu.edu. 


\title{
The Impact of Co-presence and Visual Elements in 3D VLEs on Interpersonal Emotional Connection in Telecollaboration
}

Hisae Matsui

\author{
Dissertation submitted \\ to the College of Education and Human Services \\ at West Virginia University \\ in partial fulfillment of the requirements for the degree of \\ Doctor of Education in \\ Technology Education
}

Terence C. Ahern, Ph.D., Chair

Daniel Hursh, Ph.D.

Neal Shambaugh, Ph.D.

Melissa D. Hartley, Ph.D.

John M. Oughton, Ed.D.

Department of Learning Sciences and Human Development

Morgantown, West Virginia2014.

Keywords: Co-presence, Affordance, 3D VLEs, Emotional Connection, Telecollaboration

Copyright 2014 Hisae Matsui 


\title{
ABSTRACT \\ The Impact of Co-presence and Visual Elements in 3D VLEs on Interpersonal Emotional Connection in Telecollaboration
}

\begin{abstract}
Hisae Matsui
The purpose of this study is to examine participant's perception of the usefulness of the visual elements in 3D Virtual Learning Environments, which represent co-presence, in developing interpersonal emotional connections with their partners in the initial stage of telecollaboration. To fulfill the purpose, two Japanese students and two American students were paired and participated in conversational sessions in two different virtual environments: one where they shared the environments with their partners and the other where they did not.
\end{abstract}

The participants had five twenty-minute conversational sessions in Japanese in Second Life. By following single subject research designs, the quantitative data were obtained from the results of a Likert scale, which was adapted from the measurement of social presence while the qualitative data were obtained from narrative reflections from participants and conversation analysis.

Both kinds of data were analyzed together and the following conclusions were reached: (1) learners may find avatars useful as a cue to remember the contents of the conversation, (2) 3-D VLEs may help native speakers or non-native speakers with higher proficiency to enforce emotional connections, (3) for non-native speakers, 3-D VLEs may bring positive effects, a sense of connection with their partners, and a negative effect, uncomfortableness, (4) other factors, such as topic of the conversation, gain impacts on emotional connections as the collaboration goes on. 


\section{ACKNOWLEDGEMENTS}

I would like to express my deepest appreciation to my committee chair Dr. Terence Ahern, who has had tremendous patience and expert guidance with me. Without his dedication, this dissertation would not have been possible. I would like to thank my committee members, Dr. Daniel Hursh, whose insightful suggestions guided me to reach my goal, Dr. Neal Shambaugh, whose suggestions for the prospectus determined the direction of this project, Dr. Melissa Hartley, who gave me keen advice from the beginning of this journey, and Dr. John Oughton, who gave me very helpful as well as detailed feedback, which were truly inevitable for the completion of this dissertation.

I would also like to thank my colleagues at Princeton University. Dr. Janet Temos, Director of Educational Technology Centers, guided me through my Second Life experience from the beginning and helped me tremendously in building environments for this project. I would also like to thank the Department of East Asian Studies for their generous financial support for maintaining the environments in Second Life.

In addition, I would like to thank my colleagues in the Japanese language programs, especially, Professor Seiichi Makino for his constant encouragement and his prominent knowledge and experience as an OPI tester. This project would not have been possible without partnership with Waseda University. Dr. Rika Hanamitsu from Waseda University has been a wonderful partner in this project. Her cooperation from recruiting participants to data analysis has always been highly appreciated.

Finally, I would like to dedicate this dissertation to my husband, Matt, and my son, Lukas. Their patience and support had constantly motivated me to go throughout this journey. With you, everything is possible. I love you. 


\section{TABLE OF CONTENTS}

ABSTRACT

ACKNOWLEDGEMENTS

ii

TABLE OF CONTENTS

iii

LIST OF TABLES

vii

LIST OF FIGURES

vii

CHAPTER I: INTRODUCTION

Background 1

Need for the Study and Rationale $\quad 5$

$\begin{array}{ll}\text { Purpose of the Study } & 6\end{array}$

Research Questions $\quad 6$

$\begin{array}{ll}\text { Summary } & 7\end{array}$

CHAPTER II. LITERATURE REVIEW 99

$\begin{array}{ll}\text { Introduction } & 9\end{array}$

$\begin{array}{ll}\text { Telecollaboration } & 9\end{array}$

$\begin{array}{ll}\text { Five-stage model for online collaboration. } & 12\end{array}$

$\begin{array}{ll}\text { Social Presence } & 14\end{array}$

$\begin{array}{ll}\text { Measurement of social presence. } & 15\end{array}$

$\begin{array}{ll}\text { Affordance } & 21\end{array}$

$\begin{array}{ll}\text { Virtual Environments } & 24\end{array}$

$\begin{array}{ll}\text { Second Life. } & 29\end{array}$

$\begin{array}{ll}\text { Summary } & 31\end{array}$ 
CHAPTER III: METHODOLOGY 32

$\begin{array}{ll}\text { Overview } & 32\end{array}$

Research Questions and Research Hypotheses 32

Data Collection Methodology 33

$\begin{array}{ll}\text { Participants. } & 33\end{array}$

Research design. $\quad 34$

$\begin{array}{ll}\text { Instruments. } & 35\end{array}$

$\begin{array}{ll}\text { Procedure. } & 36\end{array}$

Methods of Data Analysis. 39

$\begin{array}{ll}\text { Procedure of analysis. } & 40\end{array}$

Summary 45

$\begin{array}{ll}\text { CHAPTER IV: DATA ANALYSIS } & 47\end{array}$

$\begin{array}{ll}\text { Overview } & 47\end{array}$

$\begin{array}{ll}\text { Participants } & 47\end{array}$

$\begin{array}{ll}\text { Results } & 47\end{array}$

1. Quantitative analysis for each participant to compare two 49 environments.

2. Qualitative analysis. $\quad 49$

$\begin{array}{ll}\text { Reflections from participants. } & 49\end{array}$

Impressions after the first sessions and all of the sessions. $\quad 50$

$\begin{array}{ll}\text { Conversation Analysis. } & 55\end{array}$

$\begin{array}{ll}\text { Self-disclosure. } & 70\end{array}$

3. Integrative analysis for each participant 71

$\begin{array}{ll}\text { Jennifer } & 71\end{array}$ 
$\begin{array}{ll}\text { Mary } & 77\end{array}$

$\begin{array}{ll}\text { Yoko } & 83\end{array}$

$\begin{array}{ll}\text { Keiko } & 87\end{array}$

4. Further analysis for generalizability. $\quad 91$

$\begin{array}{ll}\text { Similarities among participants. } & 91\end{array}$

Similarity among non-native speakers. 95

$\begin{array}{ll}\text { Difference among non-native speakers. } & 95\end{array}$

Similarity among native speakers. $\quad 96$

$\begin{array}{ll}\text { Difference among native speakers. } & 97\end{array}$

$\begin{array}{ll}\text { Summary } & 98\end{array}$

CHAPTER V: CONCLUSION 99

$\begin{array}{ll}\text { Overview } & 99\end{array}$

$\begin{array}{ll}\text { Discussion } & 99\end{array}$

$\begin{array}{ll}\text { Limitations } & 104\end{array}$

$\begin{array}{ll}\text { Implications } & 105\end{array}$

Recommendations for further research 106

$\begin{array}{ll}\text { Conclusions } & 107\end{array}$

$\begin{array}{ll}\text { REFERENCES } & 110\end{array}$

$\begin{array}{lr}\text { APPENDIXES } & 128\end{array}$

$\begin{array}{lll}\text { Appendix A Survey for each session } & 128\end{array}$

$\begin{array}{lll}\text { Appendix B } & \text { Survey after the first set of sessions } & 131\end{array}$

Appendix C Survey after all of the sessions 132

Appendix D ACTFL Proficiency Guide 2012-Speaking 133 
Appendix E Graphs of social presence score for Jennifer

(Café and Individual Room)

Appendix F Graphs of social presence score for Mary (Café and Individual Room)

136

Appendix G Graphs of social presence score for Yoko (Café and Individual Room)

Appendix H Graphs of social presence score for Keiko (Café and Individual Room)

138

Appendix I Graphs of social presence score for Jennifer and Yoko

Appendix J Graphs of social presence score for Jennifer and Keiko (Café)

Appendix K Graphs of social presence score for Mary and Yoko (Café)

Appendix L Graphs of social presence score for Mary and Keiko (Individual Room)

Appendix M Graph for the number of AS-unit and self-disclosure for Jennifer (Café and Individual Room)

Appendix N Graph for the number of AS-unit and self-disclosure for Mary

144 (Café and Individual Room)

Appendix O Graph for the number of AS-unit and self-disclosure for Yoko

(Café and Individual Room)

Appendix P Graph for the number of AS-unit and self-disclosure for Keiko (Café and Individual Room)

Appendix Q Graph for the number of AS-unit and self-disclosure for Jennifer and Yoko (Individual Room)

Appendix R Graph for the number of AS-unit and self-disclosure for Jennifer and Keiko (Cafe)

Appendix S Graph for the number of AS-unit and self-disclosure for Mary and

Appendix T Graph for the number of AS-unit and self-disclosure for Mary and

Appendix U Notice of approval from IRB

Appendix V Standard adult consent form

Appendix W Flayer for recruiting participants

Appendix X List of avatars 


\section{LIST OF TABLES}

Table 1: Combinations of the participants

Table 2: Actual combinations of the participants 48

$\begin{array}{ll}\text { Table 3: Original question and its shortened version } & 71\end{array}$

Table 4: Total seconds of silence in each session 94

Table 5: Longest seconds of silence in each session $\quad 94$

\section{LIST OF FIGURES}

Figure 1: Five-stage model for online collaboration 2

Figure 2: Café setting and individual room setting 38

Figure 3. Illustration of the steps of integrative analysis for each participant 41 


\section{Chapter I: Introduction}

\section{Background}

Globalization has transformed virtually all aspects of modern life. Not surprisingly, the waves of globalization have had a great impact on foreign language education. The Modern Language Association's 2007 report on the state of language teaching in the United States recognized the importance of developing transcultural competence in a post 9/11 era.

Carlorosi, Helm, Marini-Maio and McMahon (2008) claimed that simply learning about the practices of a particular culture such as socially appropriate patterns of interaction and behavior is insufficient. It is more important to understand how "practices" and "products" are rooted in "perspectives;" such as values, attitudes, conceptions, and beliefs underlying a particular culture. In other words, the goal for the learners is to be able to view aspects of the target culture through the eyes of the members of that culture.

In the past, foreign language education provided limited opportunities for learners to engage a foreign culture due to its isolation from organic contact with the target language and its speakers outside. However, with advances in new information and communication technology, it is now possible to create direct contact between members of different cultures through virtual means. For example, one form of online language education is referred to as "telecollaboration," which involves the use of Internet communication tools such as email and chat in order to support prolonged intercultural exchanges between groups of students in various institutional settings who might otherwise not have the opportunity to interact (Belz, 2005; Belz \& Thorne, 2006). The aim for such exchanges is not merely to provide a platform for language practice, but to lead participants to develop intercultural communicative competence (ICC) (Byram 1997) through interaction and exchange (Belz \& Thorne, 2006). 
Collaborative learning, however, does not occur naturally. Salmon (2000) developed a five-stage model (Figure 1) for enabling and scaffolding remote groups to work and learn together through online networking.

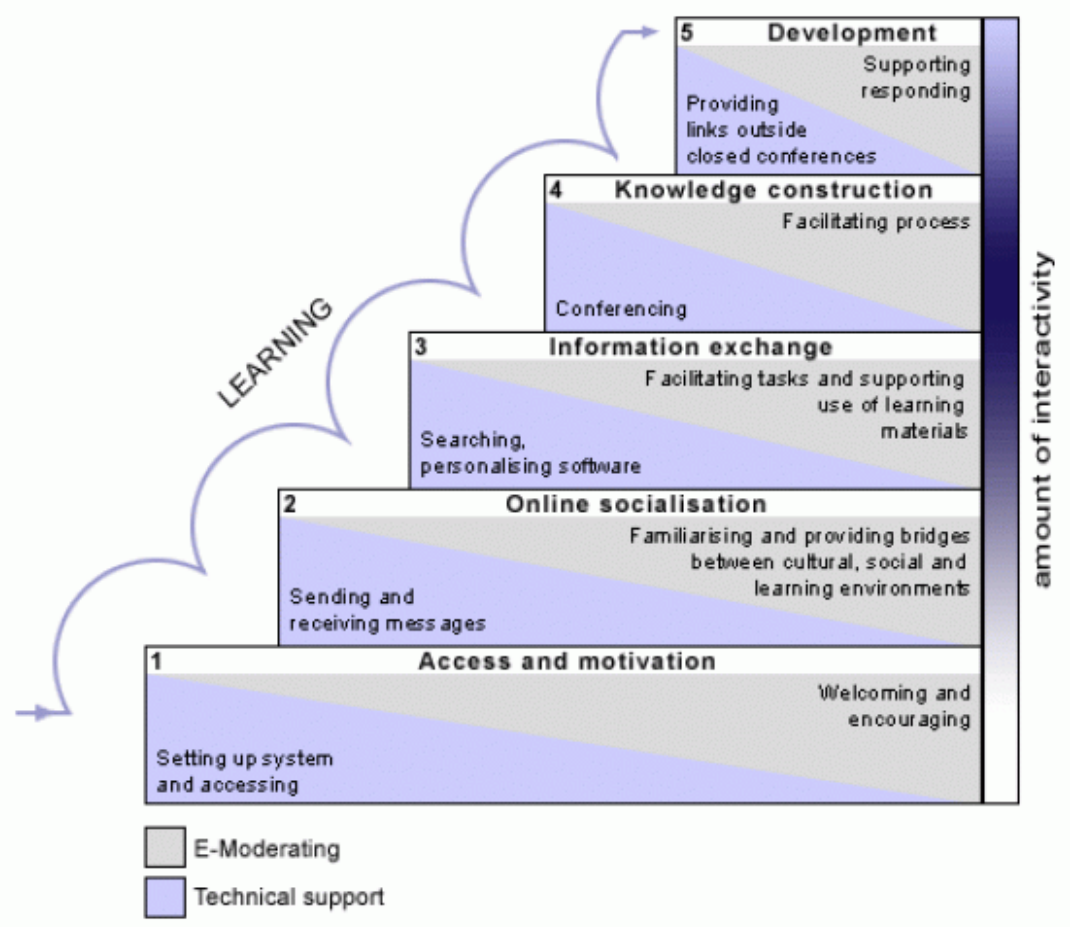

Figure 1. Five-stage model for online collaboration (Salmon, 2000)

Each stage of the model requires learners to master technical skills while it calls for different human intervention skills. Salmon (2000) pointed out that learning involves much more than a simple shift in cognition or the experience of using a computer as her underlying assumption to the five-stage model. An online learning environment offers affordances for each stage. However, online learning does not occur naturally without sensitive and appropriate design of the learning environment and the instructor's intervention (Salmon, 2000).

Affordance theory provides a view of perception and action that focuses on available information available in an environment. This sense of affordance is reflected in everyday objects, which may attract a great deal of conscious attention or none, based on individuals' 
perceptions of their affordance. This is particularly true of objects that are created by human design. These are conditioned as much by the user's knowledge, experience, and context as by its design capacities built into the technology by its designers. Affordances are an especially useful way to think about Web 2.0 applications in education, as their flexibility allows them to be utilized in emergent ways, and by different users, that may be far removed from the use cases that guided their development. Therefore, even though a designer may describe the features of an educational medium, such as learner choice, self-paced, structured index, objectively and accurately, the learner may perceive it very differently.

The way it is perceived by the learner may be very different from the designer's expectation. The discrepancy between a learner's use of an educational medium and the anticipated instructional interaction is often attributed to a weak design and implementation of appropriate technological, educational, and social affordances (Kirschner, Strijbos, Kreijns \& Beers, 2004).

Technological affordances, first of all, are the properties of the environment that are concerned with the efficient and effective accomplishment of tasks that satisfy the user's instructional intentions (Kirschner et al., 2004). Norman (2004) identifies technological affordances as the usability of an environment.

Secondly, educational affordances are those characteristics of the design that determine if and how learners exhibit a particular learning behavior within the given instructional context. In other words, educational affordances are the properties and features of the environment that stimulate, engage, and maintain collaboration between users and encourage learners to interact with the instructional content in meaningful ways aligned with the chosen pedagogy. 
Lastly, social affordances are defined as the characteristics of an online collaborative environment that "act as social-contextual facilitators relevant for the learner's social interaction" (Kreijns, Kirschner, \& Jochems, 2002). Accordingly, tools and objects in digital learning environments that possess these social-contextual properties are called social affordance devices. Learners need to trust each other, feel a sense of warmth and belonging, and feel close to each other before they will fully collaborate and recognize this collaboration as a valuable educational experience. For that reason, Salmon (2000) located "online socialization" as the second stage, which is after the individual preparation stage, in the model mentioned above. She also mentioned that even though these socialization components can gradually develop throughout the five stages, success comes with a strong foundation at stage two (Salmon, 2000). However, according to Kreijns, Kirschnerb, and Jochems (2003), there is a tendency to restrict social interaction to educational interventions aimed at cognitive processes while social interventions aimed at socio-emotional processes are ignored, neglected or forgotten. Therefore, it is crucial to consider social affordances that Web 2.0 applications may have as well as their educational and technological affordances.

Characteristics give clues to our perception of what can and cannot be done with them, that is, their sense of affordance. These clues in the environment indicate possibilities for action. Different Web 2.0 applications are likely to have different affordances for learning. Hence, in order to consider affordances that certain Web 2.0 application may have, it is necessary to determine what the characteristics of the Web 2.0 application are.

Dalgarno and Lee (2010) identified the following set of unique characteristics 3D VLEs. 1. Aspects of their representational fidelity

- Realistic display of environment 
- Smooth display of view changes and object motion

- Consistency of object behavior

- User representation

- Spatial audio

- Kinesthetic and tactile force feedback

2. Aspects of the learner-computer interactivity they facilitate.

- Embodied actions, including view control, navigation and object manipulation

- Embodied verbal and non-verbal communication

- Control of environment attributes and behavior

- Construction of objects and scripting of object behaviors

\section{Need for the Study and Rationale}

Having identified the characteristics of 3D VLEs, what are the affordances that 3D VLEs may have which contribute to successful telecollaboration? Obvious essential technological affordance in telecollaboration would be "embodied verbal and non-verbal communication" since exchanging opinions is the heart of telecollaboration. However, it is unknown how other affordances are perceived by learners as well as if these affordances can contribute to social or educational affordances.

The fourth aspect of representational fidelity that Dalgarno and Lee (2010) listed is "user representation," which involves the depiction of the user as an avatar through which he or she is able to develop and project an online identity (Dickey, 2003). This depiction of users is considered to be an important element of the fidelity of the representation because it helps create a sense of co-presence, which is defined as the sense of 'being there together' with other geographically dispersed users, in the environment, whence, it enriches the social interactions 
occurring (Schroeder \& Axelsson, 2006). As mentioned above, socio-emotional processes are needed to be paid attention to building an affective structure and communities; however, whether or not this characteristic of 3D VLEs would be perceived by learners as useful to social affordance devices is unknown.

\section{Purpose of the Study}

When new technology is introduced into educational activities, it is crucial to know not only all affordances that the piece of technology can offer, but also how these affordances would be perceived and utilized by learners. However, the newer the technology, such as 3D VLEs, is, the fewer the research on learner's perception has been done. There are previous studies on general impressions of using 3D VLEs and they suggest affordances; however, there is no research that has been done to examine learners' perceptions on one of the main affordances of 3D VLEs: co-presence.

The purpose of the study is to examine the relations between visual design elements of the environments of 3D VLEs, which represent co-presence, and interpersonal emotional connections between participants in the initial stage of collaboration.

To fulfill the purpose, the experiences of four participants were observed as they participated in conversational activities in two different virtual environments. Specifically, the perception of social presence and verbal interactions during the activities were analyzed to contemplate the possible significance for sharing environments virtually on the development of interpersonal emotional connections between participants in a telecollaborative context.

\section{Research Questions}

This study consisted of four major research questions. The research questions are: 
1. How do different visual design elements of the environments of 3D VLEs affect interpersonal emotional connections between participants in the initial stage of collaboration?

2. What is the impact that different visual design elements of the environments of 3D VLEs have on interpersonal emotional connections between participants in the initial stage of collaboration?

3. What are the possible factors that affect interpersonal emotional connections between participants in the initial stage of collaboration in 3D VLEs?

4. What are the relations between visual design elements of the environments of 3D VLEs and interpersonal emotional connections between participants in the initial stage of collaboration?

\section{Summary}

As advanced technology allows us to go beyond borders without physically relocating, the importance of ICC is greater than ever. To build ICC, knowledge of cultural customs and products is not enough. Learning about "perspectives;" such as values, attitudes, conceptions, and beliefs underlying a particular culture is crucial.

Telecollaboration makes collaboration beyond borders possible; however, simply meeting online does not guarantee that collaboration happens. The key to successful collaboration is solid foundations at the beginning of collaboration; therefore, it is important for learners to establish interpersonal emotional connections with their peers even though they are often ignored, neglected or forgotten.

Considering the social nature of 3D VLEs, 3D VLEs have potential benefits for social interactions. However, how learners perceive one of the main affordances of 3D VLEs is 
unknown. Therefore, it is imperative to examine the relations between visual design elements of the environments of 3D VLEs, more specifically sharing or not sharing environments with other participants, and interpersonal emotional connections between participants even though its designer considers it useful. This study was designed to answer the question for effective use of 3D VLEs in telecollaboration. 


\section{Chapter II: Literature Review}

\section{Introduction}

This chapter presents a detailed literature review regarding affordances of 3D VLEs in telecollaborative context. With the aim of better understanding the objectives of this study, it was imperative to carry out a comprehensive review of the related literature. Given that the study entails diverse topics, it was imperative to divide the literature review in the following subsections: 1) Telecollaboration 2) Social Presence; 3) Affordance theory, and 4) Virtual Environments.

\section{Telecollaboration}

In recent years, there has been renewed interest in bringing cultural learning to the forefront. In May of 2007, the Modern Language Association released a report entitled "Foreign Languages and Higher Education: New Structures for a Changed World" examining the state of language teaching in the US in a post 9/11 era, and giving strong support to "a broad, intellectually driven approach to teaching language and culture.” The report also emphasized "the need to understand other cultures and languages," which was identified by Daniel Yankelovich. The report included statements by Daniel Yankelovich. "Our whole culture,” they quote Yankelovich as saying, "must become less ethnocentric, less patronizing, less ignorant of others, less Manichaean in judging other cultures, and more at home with the rest of the world. Higher education can do a lot to meet that important challenge" (MLA Ad Hoc Committee on Foreign Languages 2007, pp. 1-2).

The report also included statements by Senator Daniel Akaka, who made a similar point: "Americans need to be open to the world; we need to be able to see the world through the eyes of 
others if we are going to understand how to resolve the complex problems we face" (MLA Ad Hoc Committee on Foreign Languages 2007, p. 2).

The MLA is prepared to lead the way in the reorganization of language and cultural education around these objectives. The report continued to recommend that the Foreign Language curriculum should produce "educated speakers who have deep translingual and transcultural competence," and the authors envision a future which "will situate language study in cultural, historical, geographic, and cross-cultural frames within the context of humanistic learning," and consider that more students will be motivated to continue their study of the language "if courses incorporate cultural inquiry at all levels" (MLA Ad Hoc Committee on Foreign Languages 2007, p. 4). The standards propose a systematic approach to the teaching of culture. Simply learning about "practices," such as socially appropriate patterns of interaction and behavior, or "products," which are tangible or intangible creations of a particular culture, is insufficient. It is important that learners understand how "practices" and "products" are rooted in the underlying values, attitudes, conceptions, and beliefs of a particular culture, referred to as "perspectives," and that these three are all interrelated and intertwined. Therefore, the goal for the learners is to be able to view aspects of the target culture through the eyes of the members of that culture.

An integral part of the way to becoming an intercultural speaker is intra-cultural learning, which is learning about one's own culture(s) and developing the ability to reflect on the origin of one's own beliefs and behaviors. As Byram (1997) states: “awareness of one's own values allows a conscious control of biased interpretation" (p. 35). It is true that critical cultural awareness does not require students to adapt to values of the 'target culture(s)' but rather to be aware of their own values and how they may influence their own behavior. 
In the past, foreign language education often provided limited opportunities for learners to learn culture in the sense mentioned above due to its isolation from organic contact with the target language and its speakers outside and even sometime inside of the classroom setting. Studying abroad; therefore, was the primary and one of the few options to experience another culture and to develop an intercultural awareness. However, with advances in information and communication technology, it is now possible to create that direct contact between members of different cultures through virtual means. One of the examples of the form of online language education is "telecollaboration."

Telecollaboration is a form of online language education defined by Belz (2003) as: the application of global computer networks to foreign (and second) language learning and teaching in institutionalized settings. In telecollaborative partnerships, internationally-dispersed learners in parallel language classes use Internet communication tools such as e-mail, synchronous chat, threaded discussion, and MOOs (as well as other forms of electronically mediated communication), in order to support social interaction, dialogue, debate, and intercultural exchange (p. 2).

The underlying pedagogic rationale for telecollaborative language study, according to Kinginger (2004, p. 101), is rooted in the practices of direct intercultural exchange in foreign language teaching (Freinet, 1994). The approach is further grounded in the philosophical position that language and culture are bound together in an inextricable relationship. Within such a framework, language is not conceptualized merely as a checklist of grammatical points to be isolated and transmitted to the learner and later assessed in the form of discrete-point tests, but rather as social practice itself. The goals of telecollaboration combine aspects of language, inter-cultural learning, and intra-cultural learning (Belz 2006). The main aim of telecollaboration 
is not to merely provide a platform for language practice, but to lead participants to develop intercultural communicative competence (ICC) (Byram, 1997) through interaction and exchange (Belz \& Thome 2006).

The goal of telecollaboration and, more generally, foreign language education, is no longer to produce near-native speakers (Commission of the European Communities, 2003). Instead, the more realistic and, for many, more desirable "intercultural speakers" who are, as Byram (1997) explains, able "to see and manage the relationships between themselves and their own cultural beliefs, behaviors and meanings [ ... ] and those of their interlocutors" (p. 12).

Telecollaboration offers a powerful alternative to traditional classroom-based culture learning methods, as it allows learners to interact and learn directly from actual members of the target culture while remaining in their home environment. There are various types of activities which bring together two classes in different places, such as collaborating together on the creation of websites, discussing different interpretations of film and literature, or carrying out comparative investigations on different aspects of their cultures (O’Dowd \& Ritter, 2006).

Five-stage model for online collaboration. Collaborative learning does not always occur naturally. Salmon (2000) developed the five-stage model for enabling and scaffolding remote groups to work and learn together through online networking. Each stage of the model requires learners to master technical skills while it calls for different human intervention skills. Salmon (2000) pointed out that learning involves much more than a simple shift in cognition or the experience of using a computer as her underlying assumption to the five-stage model. Stage 1 (at the base of the flight of steps) includes individuals' essential prerequisites for effective participation, access and the ability to benefit from remote group work for learning. Stage 2 involves individuals establishing a personal online identity and then finding others with whom to 
interact. At Stage 3, participants give and receive relevant and useful information about the course, and undertake course-related learning tasks. Up to and including Stage 3, a form of cooperation occurs through support by other participants for each person's goals. At Stage 4, more complex constructive tasks are possible, discussions occur, and the interaction becomes more collaborative. At Stage 5, participants look for more benefits from the system. They want help in achieving their own goals, in exploring how to integrate their online experiences into other forms of learning and in transferring and applying their learning. At this stage, sophisticated individual learning may occur that includes reflection on and transfer of knowledge.

Each stage requires participants to master technical skills, shown at the bottom left of each step. Each stage calls for different human intervention skills (e-moderating), shown at the right top of each step. The "interactivity bar" running vertically along the right of the flight of steps suggests the intensity of interactivity that can be expected between the participants at each stage. At stage 1, individuals interact only with one or two others. After Stage 2, the number of those with whom they interact - and the frequency of these interactions - gradually increases. In Stage 5 they often return to more individual learning pursuits.

Online learning offers the affordance of online socializing and networking; however, online group work will not create social interaction by itself (Preece, 2000). Sensitive and appropriate learning design and the instructor's intervention enable the socialization for learning to occur (Salmon, 2000). For that reason, Salmon (2000) located "Online socialization" as the second stage after the individual preparation stage. She pointed out that even though these socialization components can gradually develop throughout the five stages, "success comes with a strong foundation at stage two" (Salmon, 2000). 


\section{Social Presence}

The theory of social presence is perhaps the most popular construct used to describe and understand how people socially interact in online learning environments (Lowenthal, 2009). Since Short, Williams, and Christie (1976) introduced the concept of social presence, there had been quite a few numbers of definitions for the concept. Lowenthal (2009) organized these theories into three phases: phase one (1970-1980), which had been focusing on telecommunication, phase two (1980-1990), which had been focusing on Computer-Mediated Communication (CMC), and phase three (1990-current), which has been focusing on online learning. He also pointed out that most of the works which are dealing with social presence in phase three are influenced by the works of Gunawardena and Zittle (1997), Rourke, Anderson, Garrison and Archer (1999), and Tu (2002) because most researchers continue to use the instruments created by these researchers.

Although it seems like each researcher in phase three has his/her own definition for social presence, those definitions tend to fall on a continuum according to Lowenthal (2009). At one end of the continuum, the social presence is conceptualized as the degree to which a person is perceived as being "real" and being "there." One of the examples of the definitions, which has this element, would be Gunawardena's; "the degree to which a person is perceived as a 'real person' in mediated communication (Gunawardena, 1995, p. 151)." At the other end of the continuum, social presence is defined as an interpersonal emotional connection between communicators. For example, Tu and McIsaac's definition, “the degree of feeling, perception, and reaction of being connected by CMC to another intellectual entity though a text-based encounter" is clearly focused on the emotional connections among participants (Tu \& McIsaac, 2002, p. 140). On the other hand, Swan and her colleagues defined social presence as "the degree 
to which participants in computer-mediated communication feel affectively connected one to another," which also emphasized on the element of emotional connections (Swan, Richardson, Ice, Garrison, Cleaveland-Innes \& Arbaugh, 2008, p. 2).

Yamada and Kitamura (2010) brought up another perspective for categorizing the definitions; perception vs. production. One conceptualization views social presence as the perception or recognition that participants may have in the online course (Gunawardena, 1995;

Picciano 2002; Richardson \& Swan 2003; Swan \& Shih 2005; Tu, 2002; Tu \& McIsaac, 2002). The second way that social presence has been conceptualized is not as perception, but as projection (Rourke et al., 1999; Wise, Chang, Duffy \& Del Valle, 2004). Although each researcher has his/her own definition for social presence, the majority of researchers who studied social presence saw social presence as something that participants perceive. This trend has a long history, which was started from Short, Williams and Christie (1976) who introduced social presence.

On the other hand, Garrison, Anderson and Archer (2000) regarded social presence as something that a participant projects. They considered social presence as one of the three fundamental "presences," besides teaching presence and cognitive presence, which support learning. Although "perceiving” and "projecting" seem like two different things, Swan and Shih (2005) examined if there is a relationship between the two and found that participants who perceive more social presence also use significantly more social presence indicators to project their own presence to their classmates. This result indicated that there was a correlation between "perceiving" and "projecting" of social presence.

Measurement of social presence. The ways researchers defined social presence naturally influenced how they measured social presence and the conclusions they drew. 
Reserving great influences from Gunawardena and Zittle (1997), Tu (2002) and Rourke et al. (1999), researchers of social presence research have tended to either focus on participant's attitudes or online behaviors. That is, researchers who regarded social presence as something that participants may perceive, such as Gunawardena and Zittle (1997), as well as Tu (2002), focused primarily on studying participants' attitudes whereas researchers who regarded social presence as something that participants may project, such as Rourke et al. (1999), focused on online behaviors of participants.

One of the earliest researchers who studied social presence in an education setting, Gunawardena (1995), created 17 bipolar scales on a 5-point likert-type scale to measure participants' impression toward CMC (from negative to positive). This instrument was later revised by Gunawardena and Zittle (1997) and called the Social Presence Scale. The Social Presence Scale consists of 14 items that embody the concept of "immediacy" as defined in Short et al. (1976). Gunawardena and Zittle (1997) concluded that it investigated the construct of social presence more directly than the previous scale.

Tu (2002) criticized early studies on social presence, including Gunawardena and Zittle (1997)'s, for they used the same semantic differential technique as Short and his colleagues (1976) did. He argued that this technique is not an ample measure for participants' perception of social presence in CMC. He also argued that the Social Presence Scale, which was developed by Gunawardena and Zittle (1997), failed to consider other variables, such as topics, privacy and communication styles. As a result, Tu (2002) developed The Social Presence and Privacy Questionnaire (SPPQ). The SPPQ was made based on a CMC attitude instrument, which was developed by Steinfield (1986) and an instrument measuring perceived privacy, which was 
developed by Witmer (1997). The questionnaire consists of 17 social presence items and 13 privacy items on a 5-point likert-type scale.

Unlike Gunawardena and Zittle (1997) and Tu (2002), who measured social presence through a self-report questionnaire, Rourke et al. (1999) strived to measure social presence through analyzing online discussions in the community of inquiry (CoI) framework ( Garrison $e t$ al. 2000), which has been used in several subsequent studies (Hughes, Ventura \& Dando, 2007; Rourke \& Anderson, 2004; Swan, 2003). They identified three categories of social presence and 12 indicators that researchers can use to analyze transcripts of CMC and protocols for coding online discussion based on these indicators. Hughes, Ventura and Dando (2007) did a replication study for Rourke et al. (1999) and suggested several amendments particularly in the affective category. The amendments include more precise criteria for coding to reduce ambiguity, as well as some changes on categorization.

These examples show that there is still little agreement on how to measure social presence (Lin, 2004; Stein \& Wanstreet, 2003). In addition to that, even though social presence has often been presented as a perceptual construct, as Kramer, Oh, and Fussell (2006) pointed out, self-report data, which are usually collected at the end of the experiments, are retroactive and insensitive to changes in presence over the course of an interaction.

In traditional face-to-face classrooms, researchers have found that teachers' behaviors can reduce the psychological distance between teachers and students (Christophel, 1990; Gorham, 1988, Richmond, 1990; Rodriguez, Plax, \& Kearney, 1996). This perceived psychological distance between communicators is often referred to as 'immediacy' (Weiner and Mehrabian, 1968). Immediacy behaviors fall into two categories: nonverbal immediacy behaviors and verbal immediacy behaviors. Nonverbal immediacy behaviors include eye contact, 
touch, relaxed posture, and smiling (Mehrabian, 1969) whereas verbal immediacy behaviors include addressing others by name, emotional expression, acknowledgement, and self-disclosure (Gorham, 1988; Wiener \& Mehrabian, 1968). A number of studies have been performed to assess the influence of teachers' immediacy behaviors in student learning. These studies indicated that teachers' immediacy has a positive impact on students' subjective impressions about their experiences (Christensen \& Menzel, 1998; Frymier, 1994; Gorham, 1988; Moore, Masterson, Christophel, \& Shea, 1996). However, other studies showed there was no relationship between perceived teacher immediacy and actual course performance (Andersen, 1979; Andersen, Norton, \& Nussbaum, 1981).

Those immediacy studies in traditional classrooms have implications for online communication. Some researchers have argued that differing media have differing capabilities to convey the non-verbal and vocal cues that produce immediacy in face-to-face communication. Short and colleagues (1976), for example, referred to these capabilities as "social presence" and claimed that media with limited bandwidth has a respectively limited capacity to project social presence than more broadband media. They hypothesized that media-choice can be predicted such "users of any given communications medium are in some sense aware of the degree of Social Presence of the medium, and tend to avoid using the medium for certain types of interactions; specifically, interactions requiring a higher degree of Social Presence than they perceive the medium to have" (Short et al. 1976, p. 65).

The Media richness theory, which was developed by Daft and Lengel (1984), reached a similar supposition. In this theory, when information systems have the capacity to carry large amounts of information, they can reduce uncertainty and as a result, information systems that possess the capability to reduce equivocality and uncertainty possess the ability to process rich 
information (Daft \& Lengel, 1986). They argued that face-to-face communication has the highest richness whereas numeric communication has the lowest.

Likewise, Kiesler and his colleagues have referred to this characteristic as the "dramaturgical weakness" of CMC, since nonverbal behavior such as nodding, frowning or gesture articulation cannot be transmitted to interlocutors in purely text based CMC (Kiesler et al., 1984). Baron (1984) also believed that CMC at least as currently used is "ill-suited for such 'social' uses of language” and not suitable for bonding or community building (p. 136).

These researchers contended that social presence is diminished by text-based CMC because it is lacking the visual and vocalic cues that higher-bandwidth and face-to-face systems provide. However, other research results have challenged this argument (Fulk, Schmitz, \& Steinfield, 1990) or established their validity in certain specifiable conditions related to the longevity of online relationships (Walther, 1992, 1996; Walther \& Parks, 2002; Walther, Slovacek, \& Tidwell, 2001), although there were several reports of loneliness and isolation (Grubb \& Hines, 2000; Robinson, 2000).

Walther (1992) focused primarily on CMC and criticized the media deterministic view. He claimed that those studies assumed that the absence of visual cues led to an absence of sociability and the researchers who claimed media deterministic view failed to acknowledge that just as cues are filtered out, other cues are filtered into CMC. That is, CMC has some affordances that face-to-face communication does not (Walther, 1992, 1996; Walther \& Parks, 2002). He argued that the social nature of humans is the same in CMC and face-to-face environments and believed that people will find ways to compensate for any cues that are filtered out in CMC after spending enough time (Walther, 1992). 
The social information processing perspective of mediated communication, which was proposed by Walther (1992), claimed that even in strictly textual media, such as email or computer conferencing, participants are able to adjust to the restrictions of the media by adapting their language behavior to fulfill the functions of missing non-verbal cues to build interpersonal impressions, build mental models of their colleagues, and develop relationships marked by affective exchange.

The third phase of social presence research, which was mentioned in the previous section, was influenced by previous studies and theories, especially that of Walther (1992). Therefore, starting with Gunawardena (1995), the researchers in this phase, began re-conceptualizing the social presence theory moving away from a technological deterministic view of mediated communication and attached weight to personal perceptions of social presence (Gunawardena \& Zittle, 1997; Poole, 2000; Richardson \& Swan, 2001; Rourke et al., 1999). As a result, definitions of social presence began to reflect this emphasis on relational aspects of communication, including a sense of individuals' abilities to perceive others through their mediated interactions (Collins \& Murphy, 1997), focus on the degree of "tangibility and proximity" of others within a communicative situation (McLeod, Baron, \& Marti, 1997) and the degrees of affective connection (Swan \& Shih, 2005).

As seen above, social presence theory has a long history and numerous definitions as well as measurements. In spite of the fact that social presence is one of the most commonly used concepts to discuss social elements in online education, close attention is needed to be paid when the term "social presence" is used. Therefore, in this study, the term "interpersonal emotional connection" was used instead of the term "social presence" in order to avoid the ambiguousness. The measurement for the "interpersonal emotional connection" was adapted from the work done 
by Swan and her colleagues who defined social presence as "the degree to which participants in computer-mediated communication feel affectively connected one to another" (Swan et al., 2008).

\section{Affordance}

"Affordances" is a word now commonly used to describe characteristics of the learning process (Laurillard, Stratfold, Luckin, Plowman, \& Taylor, 2000). The word and concept are borrowed from the psychology of perception, where it expresses the fact that there is an internal relation between the perceiver and the perceived. Visual perception psychologist James Gibson developed "Affordance Theory" to express the relationship that exists between an animal (perceiver) and the environment (perceived) (Gibson, 1977).

Gibson's theory of perception derives from his assertion that most of our knowledge of the world around us is not simply based on our experience, but on our expectations. Affordances are the inherent uses that an object in the environment furnishes to their user, for good or ill. An object is said to afford a function to potential users and an object's complete set of affordances defines its possible functions. Therefore, affordances are a form of communication between objects and their users (Gordon, 1989). Humans tend to use objects in ways suggested by the most obvious perceived affordances, not in ways that are difficult to discover (Norman, 1993, p. 106). Affordances are neither objective properties, nor are they purely perceptual (Kuhn, 1996). Affordances capture how human beings understand what they can do with their environment. They offer clues to the environment's operation (Norman, 1988). For example, it is clearly seen that a chair affords sitting to humans; however, what is less clear is how a chair accomplishes this affordance while affording many other possible functions, such as standing and climbing, at the same time. The goal "to sit" is associated with certain properties. Humans want to sit where it 
is flat, level, knee height, and so forth. There is an acceptability range for each property; that is, the seat does not have to be perfectly flat, or exactly at knee height, for example. The invariants associated with sitting, such as flat and level, explain how humans are able to instantly adapt to a numerous number of seats, including chairs never sat on and chair designs never before seen. In addition, other invariants are associated with other possible affordances of the chair. Affordances are neither good nor bad, nor healthy or unhealthy. They are simply potential uses of an object. This sense of affordance is reflected in everyday objects and they may attract a great deal of conscious attention or none, based on the individuals' perceptions of their affordance (Cook \& Brown, 1999, p. 389).

This is particularly true of objects that are created by human design. These are conditioned as much by the user's knowledge, experience, and context as by its design capacities built into the technology by its designers. Therefore, affordances are an especially useful way to think about Web 2.0 applications in education because their flexibility allows them to be utilized in emergent ways, and by different users, that may be far removed from the use cases that guided their development (Reiser \& Dempsey, 2012). Although instructional designers are intent to design and develop digital learning environments where the design of the software makes it immediately clear to users how they can interact with and manipulate the environment, artifacts in the environment are often perceived or used quite differently than the designers' original intention (Krippendorf, 1989). According to Kirschner and her colleagues, the discrepancy between a learner's use of an educational medium and the anticipated instructional interaction is often attributed to a weak design and implementation of appropriate technological, educational, and social affordances (Kirschner et al., 2004). The social affordances must enrich the chosen pedagogy by providing engaging opportunities, which exist habitually in traditional face-to-face 
learning. Likewise, the technological affordances must not only allow these social interactions to emerge, but also ultimately facilitate them by providing an effective and efficient structure.

The educational affordances are the characteristics of artifacts in the environment that determine if and how learners exhibit a particular learning behavior within the given instructional context (Kirschner et al., 2004). In other words, educational affordances are the properties and features of artifacts in the environment that encourage learners to interact with the instructional content in meaningful ways aligned with the chosen pedagogy.

The social affordances are defined as the characteristics of artifacts in the environment that "act as social-contextual facilitators relevant for the learner's social interaction" (Kreijns, Kirschner, \& Jochems, 2002, p.13). Furthermore, tools and objects in digital learning, which possess social affordances, are called social affordance devices.

Collaborative learning environments devoid of social affordances are "likely to isolate learners from their peers" (Kirschner et al., 2004.p.51), ultimately rendering the environment little more than a simple repository of instructional content and media.

Lastly, technological affordances are defined as the characteristics of artifacts in the environment that are concerned with the efficient and effective accomplishment of tasks that satisfy the user's instructional intentions (Kirschner et al., 2004). Norman (2004) identifies technological affordances as the usability of an environment.

An online learning environment rich with educational and social functionalities is useless to teachers and learners if the usability aspect of the design was disregarded or overlooked by designers (Kirschner et al., 2004). In other words, the technological affordances of the artifacts in the environment must support the educational and social interactions. Paired with sound educational and social functionalities, efficient usability and appropriate technological 
affordances collectively determine the usefulness of online educational environment (Kirschner et al., 2004). The quality and effectiveness of online collaborative education is contingent upon the “design of, and students' engagement in, the learning environment" (Duffy \& Kirkley, 2004, p. 4). Kirschner et al. (2004) suggested that the use of appropriately designed and implemented educational, social, and technological affordances are the foundation for stimulating, engaging, and maintaining collaboration amongst learners. It is important to note that affordances are not simply tools or objects that can be developed as independent components for implementation into any collaborative learning environment (Kirschner et al., 2004). Rather, designers, teachers, and researchers must understand and embrace the relationship between users and artifacts that exhibit the aforementioned educational, social, and technological characteristics.

\section{Virtual Environments}

Virtual environments such as Second Life are not a newly introduced phenomenon. They can be trace back to the early days of MMOs (Massively Multi-player Online games) and the online role-playing games known as MUDs (Multi-User Dungeons) (Ludlow \& Wallace, 2007). With advances in computational power and network connectivity enabled MUDs to be evolved to MOOs (object-oriented MUDs), MUVEs (multi-user virtual environments), and MMORPGs (massively-multiplayer online roleplaying games) among others (Dieterle \& Clarke, 2009).

Since "virtual worlds" have been evolving, it is challenging to define what virtual worlds are. Schroeder (1996) identified creating the sensation of the user actually "being there" as the key aspect and defined virtual environments. He also identified virtual reality technologies as "a computer-generated display that allows or compels the user (or users) to have a sense of being present in an environment other than the one they are actually in, and to interact with that environment" (Schroeder, 1996, p. 25). 
Warburton (2009) categorized virtual environments into four categories to clarify their purpose and potential value as follows; Flexible Narrative (eg. World of Warcraft), Social World (eg. Second Life), Simulation (eg. Google Earth), and 3D Workspace (eg. Project Wonderland) (Warburton 2009). According to Warburton and Pérez Garcia (2009), social world such as Second Life had been receiving more attention from educators due to its more open-ended and narrative free natures.

There are several social worlds available, a few of which are Active Worlds, Twinity, Adobe Atmosphere, Smallworlds, Onvers, and Second Life. One common difference between social worlds and other online games is that social worlds usually do not have a specified set of goals for users, to achieve. In addition to that, unlike most online games, social worlds offer a situation in which users can define and establish their own environments by building or adding tools and objects as needed. After entering social worlds, users can establish goals and objectives, such as in a role-play or simulated scenario; however, social worlds themselves do not impose goals on users.

All of these applications provide an interactive 3D environment, avatars that serve as visual representations of users and an interactive chat tool for users to communicate with one another (Dickey, 2005). Residents of these virtual worlds can customize their avatars to make them look like anything they want. Most 3D virtual environments offer full customization of an avatar's appearance and gestures, allowing users to strongly identify with the chosen representation for their avatar and easily distinguish the other participants. This customization greatly influences the perceived sensation of presence and awareness (De Lucia, Francese, Passero, \& Tortora, 2009). McKerlich \& Anderson (2007) stated "the avatar characteristic is unique to MUVEs and can afford an engaging and salient educational experience" (p.38). Some 
research has shown that the use of avatars lowers inhibitions and increases social interactivity (Meadows, 2008) and the use of avatars provides participants with an added sense of immersion and presence in the virtual world (Messinger, 2008).

The use of virtual environments for experiential learning is increasingly being examined by researchers (Chittaro \& Ranon, 2007). Kalyuga (2007) found that virtual environments are highly interactive in that they provide dynamic feedback, learner experimentation, real-time personalized task selection, and exploration. Virtual environments are also often reported to have other instructional benefits, such as allowing for creativity within a rich media environment, providing opportunities for social interaction and community creation, facilitating collaboration, increasing a sense of shared presence, dissolving social boundaries, lowering social anxiety, enhancing student motivation and engagement, and accommodating millennial generation learning preferences (Jarmon, Traphagan, Mayrath, \& Trivedi, 2009).

Hedberg and Alexander (1994) suggested that their most important defining feature is the "transparent interface with which the user directly controls the objects in the context of the virtual world"(p. 215). In identifying the features of virtual environments that make them distinct from interactive multimedia, they name three aspects of virtual environments that contribute to this transparency and through which such environments have "the potential to offer a superior learning experience" (p. 218): increased immersion, increased fidelity, and a higher level of active learner participation.

There is some agreement between Hedberg and Alexander's ideas and those of Whitelock, Brna and Holland (1996), who proposed a theoretical framework in order to explore the relationship between virtual environments and conceptual learning. 
Their framework included the identification of three properties or dimensions of 3D virtual environments.

- Representational fidelity, which means increased 'fidelity'

- Immediacy of control, which means a higher level of active learner participation.

- Presence, which means increased 'immersion', Brna (1999), on the other hand, extended his earlier work with Whitelock and Holland (1996) to propose a framework that incorporates the social factors involved in the use of multiuser VEs (MUVEs).

His six-dimensional framework includes Whitelock and his colleagues'

- Representational fidelity

- Immediacy of control

- Presence

- Social fidelity

- Immediacy of discourse

- Social presence

Many authors have stressed the importance of immersion and presence, which is suggesting that these are critical features distinguishing virtual environments from other types of computer applications (McLellan, 2004; Mikropoulos, 2006; Mikropoulos \& Strouboulis, 2004).In early writings about VEs, there was a tendency to use these terms interchangeably; subsequently, debates occurred in the literature about the definitions of these terms (Slater, 1999; Witmer \& Singer, 1998).

While the sense of presence in a virtual world or environment has traditionally been used to refer to a user's perception of "being there" (Schroeder, 2002), more recent research has 
examined the concept of co-presence, which is defined as the sense of "being there together" with other geographically dispersed users. The concept of co-presence is considered to be an extension of social presence and which was included in Brna's aforementioned framework (Brna, 1999).

It is arguable that many 3D MUVEs support high levels of co-presence, owing to the fidelity or realism of the environments within which the shared sensory experiences occur and the facilities that are available for spatial and other forms of non-verbal communication (Dalgarno \& Lee, 2010). Therefore, Dalgarno and Lee (2010) considered sense of presence and co-presence as results of the various characteristics of the environment rather than being a characteristic and identified the following sets of unique characteristics of 3D VLEs.

1. Aspects of their representational fidelity

- Realistic display of environment

- Smooth display of view changes and object motion

- Consistency of object behavior

- User representation

- Spatial audio

- Kinesthetic and tactile force feedback

2. Aspects of the learner-computer interactivity they facilitate

- Embodied actions including view control, navigation and object manipulation

- Embodied verbal and non-verbal communication

- Control of environment attributes and behavior

- Construction of objects and scripting of object behaviors 
Second Life. One of the most advanced MUVEs which is widely available in Second Life. Second Life (SL) is a publicly available 3D multi-user general purpose virtual world and has been available for public access since 2003 for users over the legal age of 18. It is the conception of and is developed by Linden Lab, based in the US. SL has established itself as an attractive social, entertainment and transaction space. According to "Infographic: 10 Years of Second Life," which was published in June, 2013, an average of about 36 million new registrations are created monthly and more than 1 million users visit from around the world monthly.

As with much of other MUVEs, SL allows all participants to create content. In particular, it is possible for students, in addition to teachers, to create $3 \mathrm{D}$ objects and information content, to build learning artifacts including custom interfaces to private learning management systems, or to establish and administer groups. As such it is an environment that allows for self-governed activities of the learner (Melka-Danielsen 2008). Warburton (2009) looks into SL more specifically and identifies its eight key affordances that lend themselves to a number of learning and teaching approaches as follows; immersion, extended or rich social interaction, community presence, authentic content, identity and role play, content production tools, visualization and contextualization, and simulation.

SL was not initially intended for educational use when it was publicly released in 2003; however, it has become an important tool for instructors from over 150 academic institutions and at least 15 countries (Bowers, Ragas, \& Neely, 2009). Since SL is such a newly introduced technology and there are so many unknown factors, which are taken for granted in face-to-face interaction, SL as an educational tool is still in an evolutionary phase and faces criticism and skepticism (Bowers, 2010). On one hand, some researchers acknowledged SL's effectiveness in 
conducting group events, role-playing scenarios and virtually exploring new places (Cheal, 2007; Childress \& Braswell, 2006; Conklin 2007), and capability in realizing the constructivist potential as a rich environment for active learning. (Neely, Bowers, \& Ragas, 2010). Jarmon, Traphagan, Mayrath and Trivedi (2009) pointed out that the virtual platform of SL, especially various communication tools, provides opportunities for social interaction, collaboration, an increased sense of shared presence, partially dissolved social boundaries, and lowered social anxiety. In addition to this, interactive environments in SL suggested that a project team's internal and external communication is also likely to be fostered (Monahan, McArdle, \& Bertolotto, 2008).

On the other hand, some researchers argued that virtual worlds are a better place for play rather than learning (Foster, 2007). There are several possible barriers to use SL as an effective educational tool. Anthes (2007) pointed out that the learning curve for SL may be too steep and that technical problems with the programming may be a barrier for effective teaching. Griefing, which is defined as one player harassing another for the sake of doing so, is another problem that instructors must consider before teaching in SL (Bowers, 2010). A notable example of griefing took place in May 2007, shortly after shootings on the Virginia Tech campus, when a visiting avatar entered the Ohio University`s SL virtual campus and fired at other avatars on Ohio University‘s SL virtual campus. In the spring 2007 issue of the Indiana Law Journal reported the case that female avatars who experience virtual sexual harassment report suffering real-world anger and grief (Bugeja, 2007). These negative issues of SL must be carefully considered, especially within the context of adopting it as an educational tool (Bowers, 2010). 


\section{Summary}

The literature review in this chapter includes1) Telecollaboration 2) Social Presence; 3) Affordance theory, and 4) Virtual Environments. In the telecollaboration section, the literature review revealed the importance of ICC in this globalizing world. Telecollaboration offers learners to acquire ICC though conversations with native speakers from where they are, however, collaboration does not occur automatically. As Salmon (2000)'s five-stage model suggested, it is important to follow the stage from one to five. Successful collaboration needs a firm foundation, such as stage 2: online socialization stage. In order for better understanding of the socialization process, literature reviews on social presence has been done. The theory of social presence has a long history, varieties of definitions, as well as measurements. At the end of the section, the definition that this study adapted was introduced. In the third section, the affordance theory was introduced to examine the relationship between humans and technology. the affordance theory is adapted in various fields including education. From the point view of affordance, it is important to consider technological, educational, and social affordances to avoid discrepancies between a learner's use of an educational medium and the anticipated instruction. Finally, in the fourth section, virtual environments, as well as Second Life as one of them, were introduced as educational mediums. Previous studies suggested various affordances that virtual environments can offer as well as potential risks of using them. In the following chapter, a research method for investigating how one of the affordances that Second Life can offer will be presented. 


\section{Chapter III: Methodology}

\section{Overview}

The purpose of this study is to investigate if participants perceive the usefulness of the visual elements in 3D VLEs to develop interpersonal emotional connections with their partners in the initial stage of telecollaboration. There is one independent variable, which is whether or not sharing the virtual environment with a partner and one dependent variable: participants' perceptions of the interpersonal emotional connection. The Likert scale, which was adapted from one to measure social presence, was used to quantify participants' perceptions of interpersonal emotional connections with their partners while analysis of narrative comments from each session and conversation analysis was used to reveal if they perceive the usefulness of the visual elements in 3D VLEs.

\section{Research Questions and Research Hypotheses}

The research questions to be addressed are:

1. How do different visual design elements of the environments of 3D VLEs affect interpersonal emotional connections between participants in the initial stage of collaboration?

2. What is the impact that different visual design elements of the environments of 3D VLEs have on interpersonal emotional connections between participants in the initial stage of collaboration?

3. What are the possible factors that affect interpersonal emotional connections between participants in the initial stage of collaboration in 3D VLEs?

4. What are the relations between visual design elements of the environments of 3D VLEs and interpersonal emotional connections between participants in the initial stage of collaboration? 
While many investigators in education and psychology structure their studies to test hypotheses, an alternative is to ask experimental or research questions about those potential relations (Kennedy, 2005). By tradition, single-case experimental investigators ask experimental questions rather than the state and test hypotheses because research is viewed as a way to build an explanation of why the behavior occurs as it does in nature rather than evaluating a theory. For that reason, only the research questions are presented in this study

\section{Data Collection Methodology}

Participants. Four participants, two non-native speakers of Japanese (NNSs) from an American university and two native speakers of Japanese (NSs) from a Japanese university, participated in this study. In order to recruit participants, flyers were posted on bulletin boards, as well as distributed in the classes of the second and third year Japanese. The students in the second year Japanese were expected to be at the Intermediate-Low level and the students in the third year were expected to be at the Intermediate-Mid level in speaking in ACTFL (American Council on the Teaching of Foreign Languages) Proficiency Guidelines. To avoid gender factors on emotional connections, all participants were arranged to be the same gender, either all male participants or all female participants.

This study looked into the development of interpersonal emotional connection between participants in two different environments. Therefore, participants were paired up with his/her partner from the other university and belonged to two different pairs. As shown in Table 1 below, four pairs including two pairs for the café environment and the other two pairs for the individual room environment were formed. In this study, only four pairs were observed due to the technical and institutional constraints that were outside the researcher's control. 
Table 1

Combinations of the participants

\begin{tabular}{lll}
\hline & \multicolumn{1}{c}{ Participant C (NS) } & \multicolumn{1}{c}{ Participant D (NS) } \\
\hline Participant A (NNS) & $\begin{array}{l}\text { Pair 1: Café } \\
\text { (Participnat A and Participnat }\end{array}$ & $\begin{array}{l}\text { Pair 2: Individual room } \\
\text { (Participnat A and Participnat D) }\end{array}$ \\
C) & $\begin{array}{l}\text { Pair 3: Individual room } \\
\text { (Participnat B and Participnat C) }\end{array}$ & $\begin{array}{l}\text { Pair 4: Café } \\
\text { (Participnat B and Participnat D) }\end{array}$ \\
\hline
\end{tabular}

Research design. In this study, "single subject research designs," more specifically, "alternating treatments design" were adapted to compare the effects of two environments on the development of interpersonal emotional connection in the dyad. Single subject research designs are designs that can be applied when the sample size is one or when a number of individuals are considered as one group. These designs are typically used to study the behavioral change an individual exhibits as a result of some treatment (Gay \& Airasian, 2003). While single-subject designs have their roots in clinical psychology and psychiatry, they are useful in many educational settings (Gay \& Airasian, 2003).

There are three commonly accepted types of single subject research designs: A-B-A withdrawal design, multiple-baseline design, and alternating treatments design. While A-B-A design involves alternating phases of base-line (A) and treatment (B) and multiple-baseline design involves the systematic addition of behaviors, subjects, or settings for intervention, alternating treatment design involves the relatively rapid alternating of treatments for a single subject. Alternating treatment design is very useful in assessing the relative effectiveness of two (or more) treatment conditions (Gay \& Airasian, 2003); therefore, this design was considered to be the most suitable for this research. 
Instruments. The participants completed two kinds of surveys: one asking participants' impressions for each section and another asking their impressions of overall experiences. The survey for each session had both structured and unstructured items that include a checklist in the form of a Likert-type scale ranging from 1 = strongly disagree, $2=$ disagree, $3=$ neutral, $4=$ agree, $5=$ strongly agree, and a free response section for their brief impressions for each session. The structured part of the questionnaire had seven questions, which was adapted from the social presence scale that was developed and validated by Swan and her colleagues (Swan et al., 2008). Because the data for their study were collected from a survey for online courses, the questions that they used did not fit perfectly in this study. Therefore, the wordings of the questions were modified to fit the context of this study; however, the main idea of the original items has been kept (see Appendix 1).

In the majority of the past studies that have dealt with social presence, social presence was measured in a group; however, there are several studies that measured social presence in dyad (Abeele, Roe, \& Pandelaere, 2007; Bente, Rüggenberg, \& Kräamer, 2004; de Greef \& IJsselsteijn, 2001; Harms \& Biocca, 2004; Nowak, 2001; Nowak \& Biocca, 2003; Yamada \& Akahori, 2007). Although some of the definitions in the previous studies were referring to social presence in an online course or in a community (Picciano, 2002; Garrison et al., 2000), most of the researchers, who are considered to be influential in the area of social presence research, did not specifically exclude social presence between two people in their definitions (Short et al., 1976; Gunawardena, 1995; Tu \& McIsaac, 2002). For example, one of the pioneers of the study of social presence, Short and his colleague defined social presence as "the degree of salience (i.e., quality or state of being there) between two communicators using a communication 
medium" in their book (Short et al., 1976, p. 65); whereas Gunawardena (1995) defined it as "the degree to which a person is perceived as a 'real person' in mediated communication" (p. 151).

Given these definitions, investigating the development of interpersonal emotional connection between participants by adapting the concept of social presence perceived in dyad settings may give some implementations to the use of 3D VLEs in the initial stage of telecollaboration and to future studies, which will have more participants in each group.

The survey that was given after the first set of sessions (see Appendix 2) and one that was given after all sessions (see Appendix 3) had only unstructured items, which asked the overall impressions that participants had in the two different environments. Participants answered the questions in a narrative form in their native languages. Besides brief impressions that participants wrote on a questionnaire after each session, overall impressions were examined as well.

The surveys were prepared online by using a form tool provided by Google. A link to the online version of the questionnaire was sent to participants in Second Life and they were expected to answer the questionnaire after each session. The results from the surveys were anticipated to show transitions of perceived interpersonal emotional connection that participants would have from the first session to the last session.

Procedure. The sessions took place in a five-week period. During the period, each participant had five sessions in each group since the differentiation can often be observed with as few as five observations with each condition in single subject research designs (Gast, 2009). In each session, participants had a twenty-minute-long conversation and answered the online survey questionnaire immediately following the conversation session, which was estimated to take about five minutes. The topic of the conversation was free because this study was targeting the initial 
stage of telecollaboration, which is the "online socialization" stage in the Five-stage model for online collaboration (Salmon, 2000).

In Second Life, the representation of the self is possible through the use of an avatar. Besides basic actions that humans can do, such as walking, running, sitting, talking, and listening, an avatar can fly and teleport by using landmarks. In this study, landmarks were used to relocate avatars from one environment to another. An avatar is also capable of showing gestures; however, how to show gestures were not introduced in this project because manipulating gestures needs some practice.

Before the sessions started, the participant chose his/her avatar from sixteen pre-made avatars from the list (see Apendix X) and reported to the researcher. The researcher, then, created a Second Life account for each participant so that each avatar could have his/her preferred appearance and landmarks. Each avatar was also gaven permission to enter the session cites, which were closed to the public. Participants who did not have previous experience with using Second Life were offered a one-hour training session. In Second Life, users can modify the appearances of their avatars; however, participants were told not to modify their avatars after the first session in order to maintain the same appearance throughout the sessions.

Participants who did not have previous experience with using Second Life were offered a one-hour training session. The training included basic in-world operations, such as walking, sitting on a chair, teleporting, and talking, and how to log in and go to the assigned meeting place. Since the random order for the different treatments was important in alternating treatments design to increase the likelihood that the observed effects were the result of the treatment rather than some extraneous influence, the order of the sessions was random as long as the participants' 
availabilities permitted. The protocol of the study was reviewed by Institutional Review Board (IRB) through the expedited review procedure before collecting the data (see Appendix U).

The virtual environment in Second Life was used. A 4096 sq. m (64 x 64, 938 prims) area, which had been rented from NMC (New Media Consortium), was used for this study. The land had two settings; the café setting, which was for the experience of sharing an environment, and the individual room setting, which was in the experiment of not sharing an environment (see Figure 2).

\section{Café setting}
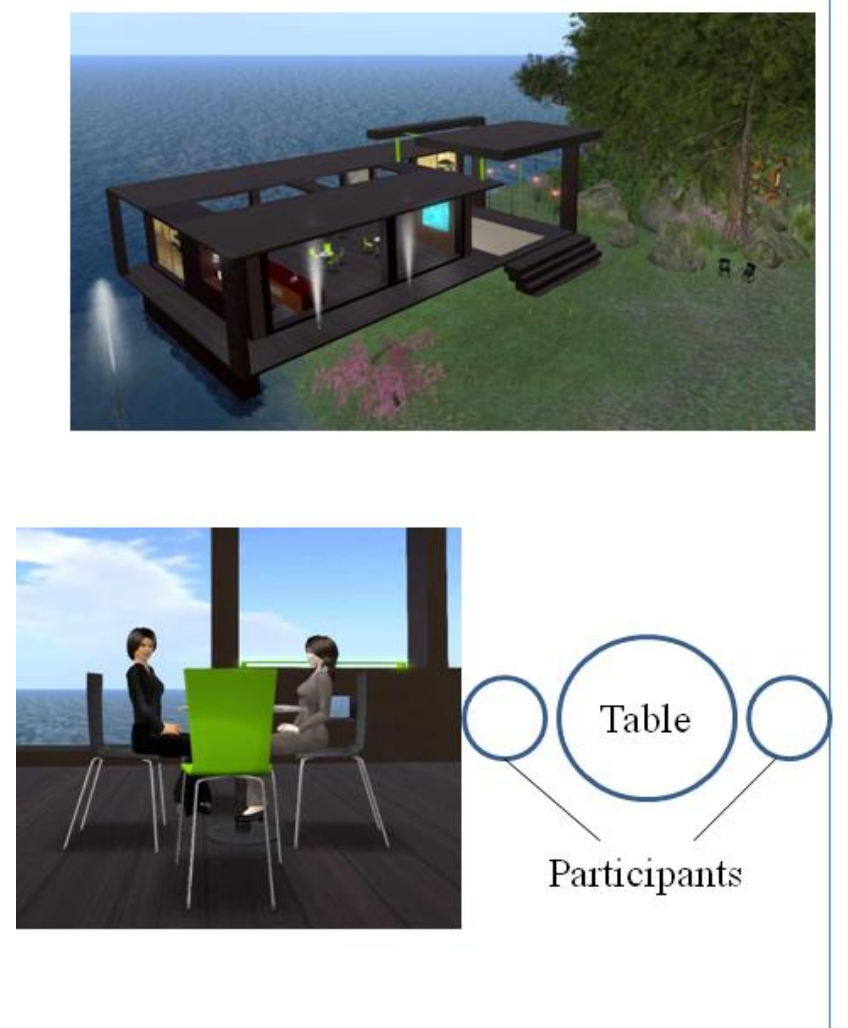

\section{Individual room setting}

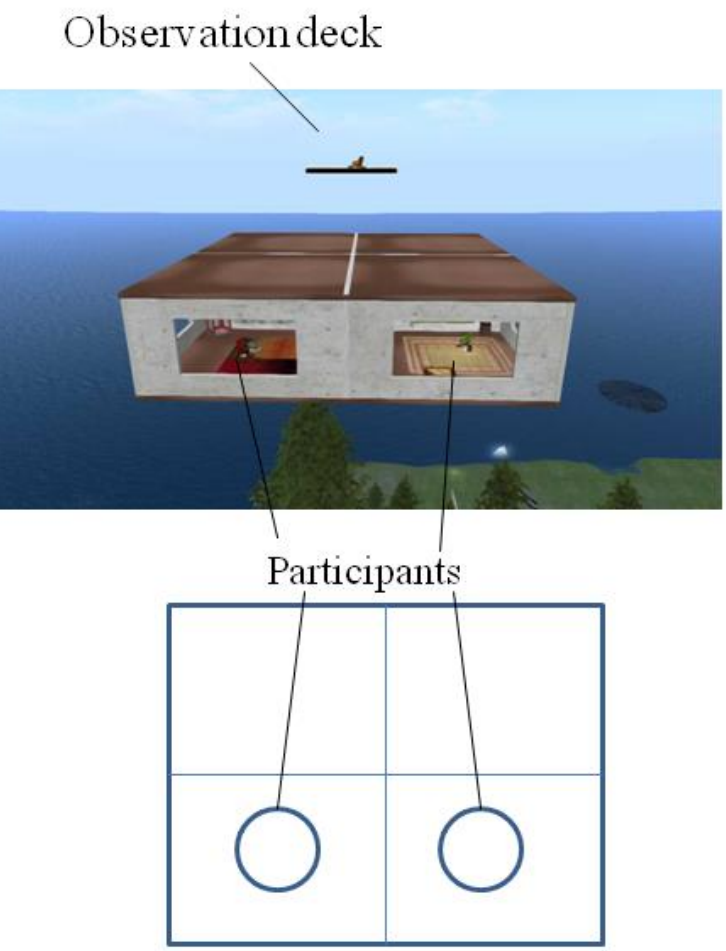

Figure 2. Café setting and individual room setting

Each participant received a folder of landmarks for the locations where sessions would take place and he/she could go to the location from anywhere by clicking the landmark. The 
environment was closed to participants so that no other users would interrupt the sessions.

During the sessions, participants communicated by using voice chat, which is an interactive communication application. In Second Life, one can choose if he/she hears voices of others from the avatar position or camera position. In this study, all of the participants set it so that they would hear the sound from their avatars' positions.

In the café setting, participants sat around a table so that they would able to see their interlocutors' avatars and sit close enough to hear their interlocutors' voice. In the individual room setting, although they could not see their interlocutors' avatars, they could still hear their interlocutors' voice because their rooms were located next to each other. Participants were free to do anything except for looking at his/her partner's avatar by controlling camera view.

All of the participants' interactions, both video and audio, were recorded with Fraps, a real-time video capture program for conversation analysis. In both environments, the researcher logged in with the participants for recording and supporting purposes; however, her avatar was out of sight of the participants by hiding under the water in the café environment or staying on the observation deck, which was located above the individual rooms.

Methods of Data Analysis. In single subject research designs, these three analyses are often used to analyze the data: visual analysis, statistical analysis, and qualitative analysis. Each analysis has its own particular advantages and limitations; therefore, by using these methods in combination and increasing the amount and types of data collected, the researcher likely enhances the ability to demonstrate a functional relationship (Richard, 1999). For that reason, three perspectives to investigate the development of interpersonal emotional connection between participants were used in this study by adapting a mixed methods research design: examining the transitions of perceptions of interpersonal emotional connections, analyzing the content of the 
conversation and examining reflections after each session as well as overall impressions from the participants.

The approach of data analysis in a mixed methods research combines both quantitative and qualitative forms of inquiry and allows a comprehensive understanding of the research problem through the collection and analysis of multiple sources of data (Creswell, 2009). In this study, "concurrent embedded strategy" of the mixed methods approach was chosen for the complex analysis. "Concurrent embedded strategy" is identified by its use of one data collection phase, during which both quantitative and qualitative data (Creswell \& Plano Clark, 2007) are collected simultaneously but has a primary method that guides the project and a secondary database that provides a supporting role in the procedures.

Procedure of analysis. By following "concurrent embedded strategy," there are four stages of analysis for this study.

1. Quantitative analysis for each participant to compare two environments. The purpose of the quantitative data analysis was to examine the transition of perceptions of social presence that participants had from the first session to the last session. To quantify the perception that a participant had after each session, ordinal responses from the survey were scored using the scale (1=Strongly Disagree) to (5=Strongly Agree). The mean score for the seven items, as well as each item on the survey from each session, were illustrated on a line graph in the order of sessions, from the first to the last. In order to compare the interpersonal emotional connections that each participant perceived in the two different environments, the results from the two environments from each participant was displayed on the same graph, which is illustrated in Figure 3 below. Thereafter, the graph was examined by visual inspection, which is a typical way of data analysis in single-subject research (Gay \& Airasian, 2003). 


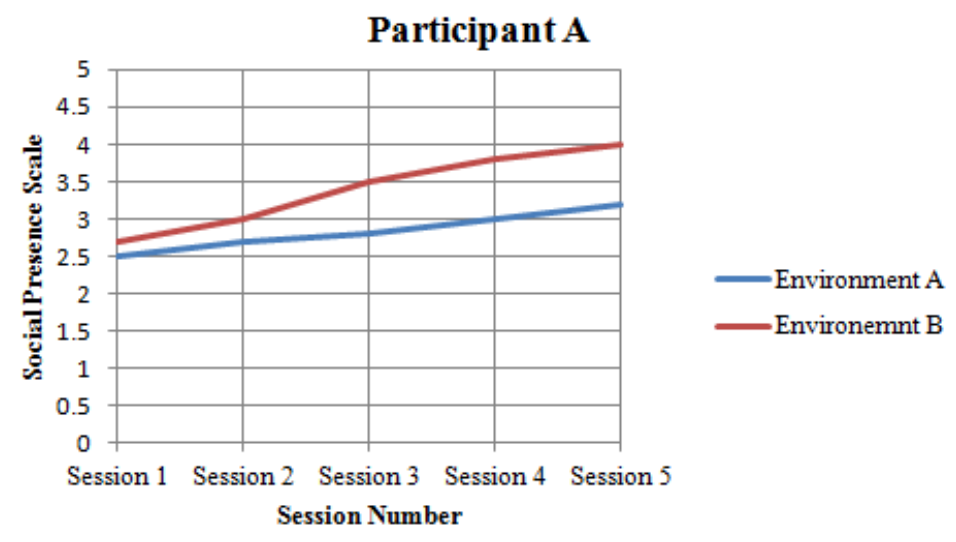

Figure 3. Sample of the graph for each participant

2. Qualitative analysis. In order to investigate how participants actually communicated in each environment, and what their reflection were, qualitative analysis was conducted. Accordingly, there are mainly two types of qualitative analysis: Conversation Analysis (CA) of verbal interactions and analysis of reflections from participants.

The contents of the verbal interactions between participants were closely examined by following CA to reveal how different visual design elements of the environments affect verbal interactions that are manifestations of interpersonal emotional connections. All interactions during the sessions were transcribed fully for content and were analyzed.

Considering having less non-verbal cues and cultural differences, the verbal interactions were examined from the perspective of self-disclosure. This indicator, which is considered to be more universal among cultures (Chen \& Nakazawa, 2012), is chosen from various closeness indicators that Nakayama (2008) presented.

Self-disclosure refers to the process by which one person verbally reveals information about the self, including demographic information, thoughts, feelings, and experiences to another person (Derlega, Metts, Petrinio, \& Margulis, 1993). Self-disclosure ranges from superficial information, such as name, age, and education, to intimate information, such as deepest fears and 
most private fantasies. It can occur between strangers or people who have close and personal relationships with each other. Self-disclosure is one of the key behaviors used to initiate, develop, and maintain relationships (Dindia, 2000). To measure self-disclosure, The Jourard Sixty-Item Self-Disclosure Questionnaire, which was developed by Jourard and Lasakow (1958), has gained popularity. This questionnaire asks research participants to rate the amount of talk they have previously disclosed about a series of topics, such as "my personal views on communism"or "how much money I make".

Antaki, Barnes and Leudar (2005) criticized the research design because they believed it is "misleadingly simple" (p. 182). They pointed out that self-disclosure is something people do in some contexts. Topic alone is not enough to determine whether something is self-disclosure. In order to find what determines self-disclosure and "how the circumstances of its bringing-off will color what it does in the interaction" they adapted Conversation Analysis (CA) (p. 183). CA was used in the qualitative research to "grapple with some small bit of the world in order to get an analytic handle on how it works" (Sidnell, 2010, p. 1). CA's objective is to crack the underlying "machinery" (Sacks, 1984, p. 26) that powers social interaction by sticking close to the data (Sidnell, 2010) and by beginning with "why that now?" Since knowing what determines a selfdisclosure was quite variable in this study, CA was adapted to examine self-disclosures during sessions.

First, the recordings of all of the sessions were transcribed. Two coders separately refined the scripts into the Analysis of Speech Unit (AS-unit), which was defined by Foster, Tonkyn and Wigglesworth (2000). They defined an AS-unit as “a single speaker's utterance consisting of an independent clause or subclausal unit, together with any subordinate clause(s) associated with it" (Foster et al. 2000, p. 365). Although the AS-unit was originally made to analyze English speech, 
Kikuchi (2012) adapted the AS-unit to analyzed Japanese speech. In this study, the rules that Kikuchi established in his paper were applied to judge the AS-units of the transcripts.

After all transcriptions were marshaled into AS-units, unnecessary parts were omitted by following the guideline suggested by Foster et al. (2000). They suggested three levels of application: level one: to be used for full analysis of all the data; level two: to be used for highly interactional data; and level three: to be used when analysis of non-fragmentary is necessary. Considering the purpose of Conversation Analysis, level two was adapted for this study. In level two, one-word minor utterances, such as "Yes," "No," and "Uhuh," and echoic responses were excluded. Following Kikuchi (2012)'s guidelines, additional exclusion, AS-units that include untranscribable parts due to sound problems, was applied besides level two guideline by Foster et al. (2000). The two coders then reconciled their differences and finalized one set of coding data.

The coding data were determined as self-disclosure or others by two coders separately. They first determined if each AS-unit was self-disclosure or not by following the two features, which were claimed by Antaki et al. (2005). They are that the utterance has to be "of something said 'on one's own behalf" (p. 186) and "designed to be understood as volunteered"(p. 187. ) Since the levels of the self-disclosure was also determined, the other feature, the utterance has to be "significant, dramatic or newsworthy in the circumstances of the interaction" was not applied for the determination.

The AS-units that were determined as self-disclosure were further rated to see their level of self-disclosure by following the method that Tidwell and Walther used. (Tidwell \& Walther, 2002). They adapted the three layer categorization scheme of Altman and Taylor (1973) to assess the levels of self-disclosure. Altman and Taylor (1973) presented "social penetration theory," 
which refers to (1) overt interpersonal behaviors, which take place in social interaction, and (2) internal subjective processes, which precede, accompany, and follow overt exchange (p. 5). They also suggested three layers; peripheral, intermediate, and core layers, to describe the depth of the interpersonal relationship.

The peripheral layer (level one), which is concerned with biographic data, the intermediate layer (level two) deals with attitudes, values, prejudices, opinions, aspirations, dreams and desires, and the core layer (level three) is comprised of highly personal aspects related to basic values, beliefs, needs, fears, self-concept, emotions, feelings and things people are ashamed of. The utterances of each layer included: "I am a student (peripheral layer)," "I like Disney movies (intermediate layer)" and "I felt guilty about cheating on the test(core layer)." After the levels of self-disclosure were determined, the two coders reconciled their differences and finalized the final levels.

Besides CA, analysis of reflections was conducted to examine the possible factors that affect interpersonal emotional connections. For that purpose, the narrative part of the questionnaire asked specifically if the participants thought they had more or less of an emotional connection with their partners than the last time and what made them feel that way to clarify the factors that participants perceive, In addition to that, narrative questions that ask participants to compare the two environments were also given after having their sessions in the both environments for the first time as well as after all of their sessions.

3. Integrative analysis for each participant. After the quantitative and qualitative analysis, the results were combined to examine each participant's perceptions and the effective factors for the perseption by following concurrent embedded strategy of the mixed methods approach. Results from survey for social presence score were treated as a primary method, which 
guided the analysis and all other data were treated as secondary database, which provided supporting roles in the procesures as Figure 3 shows below.

4. Further analysis for generalizability. One of the major criticisms of single-subject research studies is that the results cannot be generalized to a population of interest. However, with replication, generalizability is expected to be increased. Therefore, results from each participant was compared with other participants to see if there were any tendencies in the results. Furthermore, these results were also compared among non-native or native speakers of Japanese to examine if there were any tendencies in a similar cultural and language background.

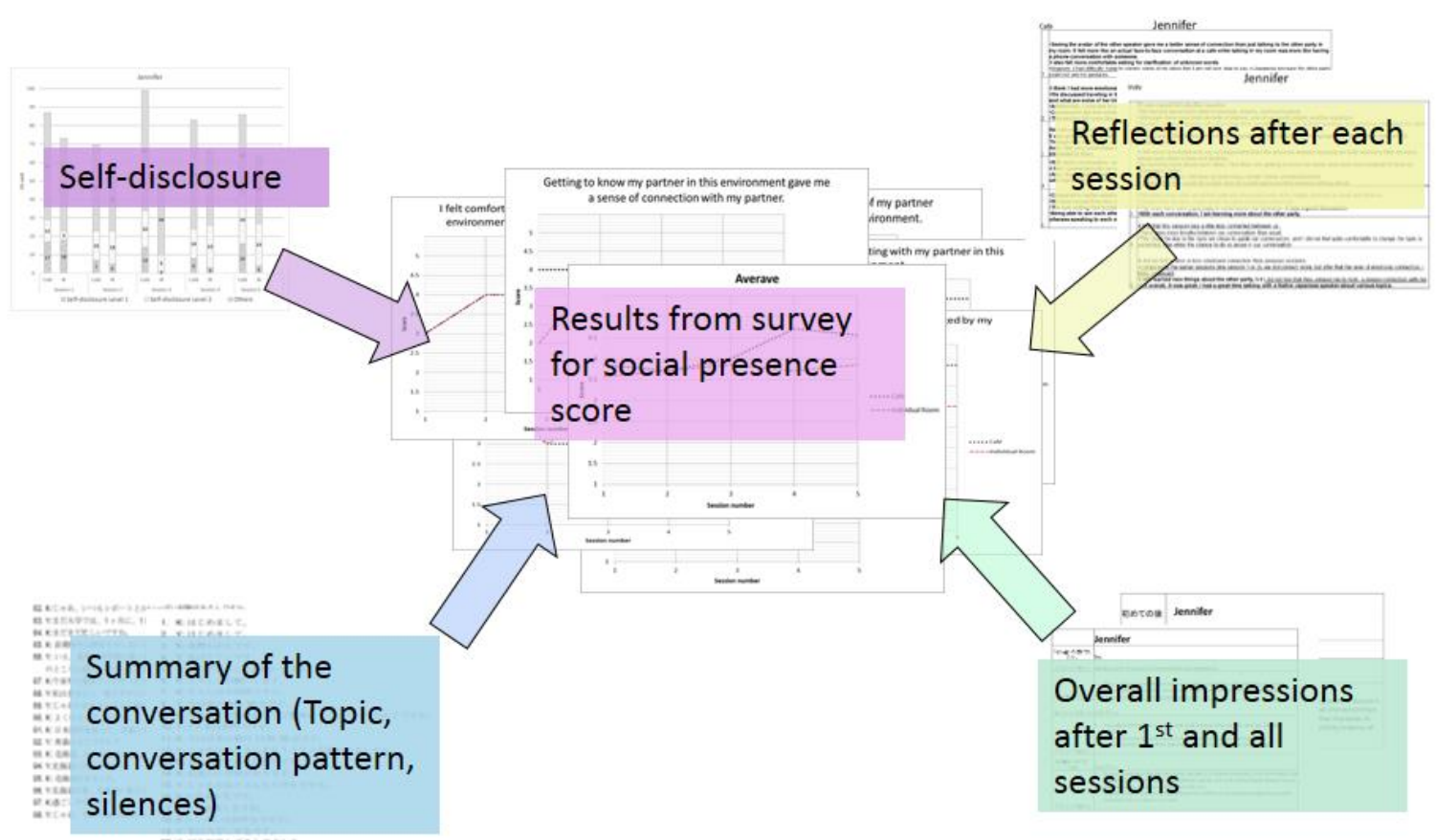

Figure 3. Illustration of the steps of integrative analysis for each participant

\section{Summary}

In this chapter, the methodology and procedures, which were used in this research, were presented. The survey included the following parts: demographics, social presence and a brief impression of each session. The quantitative study was conducted to investigate the development 
of participants' perceptions of interpersonal emotional connections by using the social presence score, which was developed and validated by Swan and her colleagues (Swan et al., 2008). Analysis of reflections from participants and conversation analysis was conducted for the qualitative study to examine participants' perceptions of the usefulness of the visual elements in 3D VLEs. An Institutional Review Board (IRB) review was conducted before collecting the data. Participants for this research were undergraduate students: two native Japanese speakers who were studying at a private Japanese university and two non-native speakers who were studying Japanese at a private university in the United States.

Conversation sessions were held in Second Life and the survey was distributed and collected by a form tool provided by Google. Once the quantitative data were collected, the data were converted into a table using Microsoft Excel and then analyzed. Furthermore, the quantitative data were compared with qualitative data to discuss if participants perceived the usefulness of the visual elements in 3D VLEs to develop interpersonal emotional connections with their partners. 


\section{Chapter IV: Data Analysis}

\section{Overview}

The purpose of this study is to investigate if participants perceive the usefulness of the visual elements in 3D VLEs to develop interpersonal emotional connections with their partners in the initial stage of telecollaboration. The aim of this chapter is to provide a detailed description and analysis of the data collected from two native speakers of Japanese and two nonnative speakers of Japanese. The data collected in this study were utilized to address the following questions:

- RQ1- How do different visual design elements of the environments of 3D VLEs affect interpersonal emotional connections between participants in the initial stage of collaboration?

- RQ2-What is the impact that different visual design elements of the environments of 3D VLEs have on interpersonal emotional connections between participants in the initial stage of collaboration?

- RQ3-What are the possible factors that affect interpersonal emotional connections between participants in the initial stage of collaboration in 3D VLEs?

- RQ4-What are the relations between visual design elements of the environments of 3D VLEs and interpersonal emotional connections between participants in the initial stage of collaboration?

\section{Participants}

Two non-native speakers of Japanese, Jennifer and Mary, and two native speakers of Japanese, Yoko and Keiko participated in this study. The participants were paired up and assigned environments for sessions as below. 
Table 2.

Actual combinations of the participants

\begin{tabular}{|c|c|c|}
\hline & $\underline{\text { Yoko (NS) }}$ & $\underline{\text { Keiko (NS) }}$ \\
\hline Jennifer (NNS) & $\begin{array}{l}\text { Pair 1: Individual room } \\
\text { (Jennifer and Yoko) }\end{array}$ & $\begin{array}{l}\text { Pair 2: Café } \\
\text { (Jennifer and Keiko) }\end{array}$ \\
\hline Mary (NNS) & $\begin{array}{l}\text { Pair 3: Café } \\
\text { (Mary and Yoko) }\end{array}$ & $\begin{array}{l}\text { Pair 4: Individual room } \\
\text { (Mary and Keiko) }\end{array}$ \\
\hline
\end{tabular}

Jennifer and Mary (NNSs). Jennifer and Mary are female students from an American university. They both studied the first year level of Japanese at the university and they both were taking the second year level of Japanese when they participated in this study.

Background of Jennifer. Jennifer is a 20 -year-old female student. She has Chinese parents and came to the United States when she was ten years old. Before she and her family came to the United States, they had lived in Japan for two years. Therefore, even though she was taking the second year Japanese when she participated in the project, her proficiency level of Japanese language and cultural knowledge of Japan were higher than her peers in her class. Her level of speaking proficiency was assessed by a fully certified ACTFL tester of Japanese and her level on the ACTFL Oral Proficiency Interview (OPI) was "Intermediate-High" (See Appendix D for the description).

Background of Mary. Mary is a 20 -year-old female student. She also has Chinese parents and she and her family came to the United States when she was four years old. Unlike the Jennifer, she has never been to Japan and has had limited contact with native Japanese speakers. Her level of speaking proficiency was also assessed by the tester and her level on the ACTFL Oral Proficiency Interview (OPI) was "Novice-High" (See Appendix D for the description). 
Yoko and Keiko (NSs). Yoko and Keiko are female students from a Japanese university. They both are senior students and their specialization is Intercultural Communication. Yoko was 21 years old and Keiko was 22 years old at that time.

\section{Results}

\section{Quantitative analysis for each participant to compare two environments. To} quantify the perception that a participant had after each session, ordinal responses from the survey were scored using the scale ( $1=$ Strongly Disagree) to (5=Strongly Agree).

The mean score for the seven items on the survey and score from each item from each session were illustrated on a line graph in the order of the sessions, from the first to the last. In order to compare the elements of interpersonal emotional connections that each participant perceived in two different environments, the results from two environments from each participant were displayed on the same graph, which is illustrated in Appendix E-H.

The data were also arranged by each pair to compare and contrast the perceptions of each participant in the pair. The results from each pair are illustrated in Appendix I-L.

The graphs were examined with other results to answer research questions under "3. Integrative analysis for each participant" below.

2. Qualitative analysis. Two types of qualitative analyses, analysis of reflections from participants and Conversation Analysis, were conducted. The results were examined with line graphs, which were shown in the previous section, in "3. Integrative analysis for each participant"

Reflections from participants. The narrative parts of the surveys after the first sessions and after all of the sessions were summarized by each participant to examine their first impressions and the impressions that they had after all of the sessions. These impressions were 
combined with other results for the further analysis in "3. Integrative analysis for each participant.'The narrative parts of the survey for each session were extracted and were analyzed with line graphs which show the development of the participants' perceptions of interpersonal emotional connections in "3. Integrative analysis for each participant."

Impressions after the first sessions and all of the sessions. The results from two survey questions, which asked participants to compare their sessions in the two environments, were summarized by each participant below. Further analysis will be conducted later in the chapter.

Jennifer. For the question, "did you find any difference between the conversations in the café and that in the individual room?" she answered "No" after the first sessions; however, her answer changed to "Yes" after all of the sessions. She listed her partner's personalities and the experiences that she had as factors that made her to think so. That is, for her, the environments did not have impact on the conversations. She looked back on the sessions in both environments and had impressions that she briefly talked about a variety of topics in the café environment while she talked at length about a limited number of topics in the individual room conversation. It gave the impreseion she had detailed conversations in the individual room; however, she mentioned that she "related more with the conversation in the café."

For the question "which environment would you prefer to get to know someone you've never met in real life?" Jennifer answered "café" both after the first sessions and after all of the sessions. She mentioned that she felt that seeing another person's virtual character has "a stronger sense of personal interaction" than just speaking to someone without seeing the other character. She also described her experience as meeting in the café had "more authenticity in terms of reallife interactions." After all of the sessions, her preference was based on the impressions that in the café environment, one can meet with a person "face to face" and he/she feels "less pressure." 
She added getting to know someone is not only just having a conversation, but also including "reading their expression, gestures, and other body language" even though she did not use any gestures nor body languages in Second Life.

Lastly, for the question "which environment do you think you would feel more comfortable if you would have to discuss deeper issues, such as your values, attitudes, conceptions, and beliefs underlying your culture, with someone you've never met in real life?"she also answered "café" after all of the sessions. Jennifer listed two points as reasons for the preference: expectation for the good behavior and the sense of innate safety. She thought that since one is in the café environment, which is more like being with real people, it is more likely that both parties will be "on their best behavior and be civil and cordial about deeper issues," even when the partner disagrees with him/her. For the second point, she explained that the sense of innate safety would "boost" her "confidence to discuss deeper issues" with someone she does not know very well.

Mary. For the question "did you find any difference between the conversations in the café and that in the individual room?" she answered "yes" both after the first sessions and after all of the sessions. After the first set of the sessions, she realized that was "more engaged" in the conversation in the café and "more comfortable" in talking in the individual room. In the café environment, since she felt the conversation was more like talking to someone in person, she was "very invested in" continuing the conversation and expressing herself. She admitted that she even used gestures in front of the computer even though she knew her partner could not see her. She also realized that pauses and mistakes in the conversation made her feel "a great deal more" in this environment than in the individual room environment. She even mentioned that she felt that while her partner for the café environment was much more willing to talk, she was more 
willing to talk in the individual room environment. These comments shows how great the impact of the environments was on her emotional states during the sessions.

After all of the sessions, she felt differences in the style of conversation. For her, the café environment felt "more like an interview setting;" as a result, there was "more pressure to keep talking and the conversation felt strongly unidirectional." She also felt pauses were "a lot more awkward" in the café, so one of them would "usually try to jump in with a topic, not necessarily very connected to the last topic." In addition to these, she felt that the conversation was "usually dominated" by one of them.

On the contrary, she felt the conversation in the individual room environment was "more natural and bidirectional." She felt that even though her partner spoke less than her or her other partner in the café, the conversation was "more bidirectional," as she and her partner "seemed to switch back and forth more often." She also noticed that the pauses in the conversation "felt less awkward" since there seemed to be "less pressure to continue speaking for a long time." For her, the transitions between the topics of conversation seemed "more natural," since both of them "worked together to steer the topic of the conversation" in the individual room environment. Even though the environment may be a part of the reasons why she felt as mentioned above, it is also more likely that the personalities of conversation style of her partners affected her impressions.

Mary answered "individual room" for the question "which environment did you prefer to get to know someone you've never met in real life?" both after the first sessions and after all of the sessions. When looking back on her experiences of meeting new people for the first time, she preferred the individual room because she felt more comfortable in that environment. While she felt more engaged in the conversation in the café, she felt a lot less comfortable pausing or 
recovering from speech mistakes in the café as mentioned above. She also mentioned that if she were trying to get to know someone she has never met before, she would prefer to be "slightly less self-conscious" so that they can get to know each other better.

Her preference remained after all of the sessions. She noticed that with her partner in the café, while she felt that she and her partner got to know a lot more about each other because they both spoke more, she did not feel "quite as comfortable sharing or listening to personal details" because she felt "a bit disconnected" from the conversation and partner. On the other hand, with the individual room partner, though they both shared "less information," she felt that she remembered more of the information and she "used it to steer the conversation."

Lastly, she also answered "individual room" for the question "which environment do you think you would feel more comfortable if you would have to discuss deeper issues, such as your values, attitudes, conceptions, and beliefs underlying your culture, with someone you've never met in real life?"

Yoko. For the question "did you find any difference between the conversations in the café and that in the individual room?" she answered "yes" for both after the first sessions and after all of the sessions. Looking back on her first experience in both environments, she had an impression that once she became accustomed to Second Life, meeting someone in the café environment was just like meeting someone face-to-face. On the contrary, in the individual room environment, she often felt like she was talking to herself and losing her attention to her partner in the individual room because she could not find an "object" to talk to.

After all of the sessions, she had the same preference and explained the reason as even in virtual environments, the ease and impression of the interactions were largely affected by whether or not the partner's avatars were visible. She felt like she did not have any cues, which 
would remind her of the content of the conversation that they had in the previous sessions in the individual room environment compared to the café environment. She also felt like she was simply throwing her words to the wall; therefore, she felt unsure of their conversation and it was hard for her to realize that she was interacting with her partner. She pointed out that when we try to recall a person whom we have met, we tend to recall the person's face and his/her clothes. However, in the individual room, since she did not have the "core" recall factors, she felt like her memory of her partner was vague.

For the same reasons that she listed in the previous question, she answered "café" for the question "which environment would you prefer to get to know someone you've never met in real life?" After the first sessions, she added the point that she felt it was easier to make an image of her partner with the help of appearance that is an avatar in this case. After all of the sessions, she also mentioned that if she would meet someone for the first time and build a relationship, it is easier when she can see the person's face because she would feel more at ease.

In spite of the preference for the café environments shown above, she answered “environment didn't matter" for the question "which environment do you think you would feel more comfortable if you would have to discuss deeper issues, such as your values, attitudes, conceptions, and beliefs underlying your culture, with someone you've never met in real life?" She explained the reason as she thought the time that participants spend with their partners would be more crucial than the environment if they discuss matters that are more serious. She looked back on her sessions and noticed that she was gradually able to talk with her partners frankly with each session in both environments. She also pointed out that even in face-to-face conversations, whether or not she could talk about the serious issues depended on the person whom she was talking to, that is, personality and compatibility would play a larger role. No 
matter what kind of environment she would be in, she felt it would be very difficult in the initial stage of the relationship especially with people from a different country.

Keiko. She answered "yes" to the question "did you find any difference between the conversations in the café and that in the individual room?" both after the first sessions and after all of the sessions. She explained the reason after the first sessions that whether or not she could see her partner's avatar affected her consciousness to her partner. After all of the sessions, she explained that she had a feeling that "something was wrong" in the environment where she was not able to see her partner's face. She also mentioned that a sense of anxiety of whether her partner was there or not was depending on whether she could see her partner's avatar or not.

She also answered "café" for the question "which environment would you prefer to get to know someone you've never met in real life?" both after the first sessions and after all of the sessions. After her experiences in both environments for the first time, she found it was easier to talk to her partner when she could see her even in the form of an avatar. After all of the sessions, she mentioned that although avatars were mere virtual figures online, she still felt that avatars show how her partners express themselves. The same as Yoko, she also answered "environment didn't matter" for the question "which environment do you think you would feel more comfortable if you would have to discuss deeper issues, such as your values, attitudes, conceptions, and beliefs underlying your culture, with someone you've never met in real life?" She explained that she was able to talk about the view of her own culture in both environments; therefore, she thought the environment did not matter.

Conversation Analysis. In order to examine the content of the conversations, first, main topics, flow of the conversations, and issues that arose during each session were summarized. 
Secondly, self-disclosures that appeared during the sessions were determined and levels were given to the self-disclosures.

Summary of the conversation. Jennifer and Yoko - Session 1 at Individual Room. Since this was the first session for this pair, they mainly exchanged their basic information such as academic year, academic major, upbringings, places that they have been to in Japan, hobbies, interests of studies, and plans after graduation. Although they talked about various topics, there were some topics that had an extended length of the sequences. Such topics included places where both of them were familiar with and Jennifer's research interest. The conversation sequence was mainly one of them asking a question and then the other answering. There were two long silences: one 6-second silence and one 14-second silence.

Jennifer and Yoko - Session 2 at Individual Room. There were some minor technical problems during the session; however, overall the interactions were understandable and Jennifer and Yoko carried out the session fairly well. The conversation started with current weather then shifted to their favorite seasons. After that, they asked each other what they did during the last weekend and that led to a fairly long sequence about Disney movies and Disneyland then amusement parks in general. The topics were very cohesive and there were not many jumps between topics during the session. There were four silences during the session: one 4-second, 6second, 9-second silences and a long silence, which lasted for 15 seconds.

Jennifer and Yoko - Session 3 at Individual Room. The first minute of the conversation was missing because they started the conversation before recording was ready. When the recording started, they were talking about things that they do on Halloween in both countries. Then the topic shifted to other annual events, such as Christmas, Valentine's Day and New Year's Day. There were some personal views involved in the sequence; however, they mainly 
talked about general customs in their countries rather than exchanging personal experiences on these events. After the segment, the topic shifted to living situations during the college, such as living in a dorm or an apartment. During the segment, even though they explained general living situations for college students in their countries, there were significantly more personal views, such as inconveniences of living in an apartment, how expensive to live near campus. There were seven silences during the session: one 5 and 11-second silences, and two 7 and 8-second silences, and a very long silence, which lasted for 20 seconds.

Jennifer and Yoko - Session 4 at Individual Room. Yoko started the conversation by asking Jennifer how Thanksgiving was. After Jennifer answered the question, the topic shifted to food that people eat during the holiday season in both countries. The content of the conversation was mixed with general information and personal preferences. Then the topic was switched to driver's licenses. The rest of the session, which was approximately three-fourths of the session, was dominated by topics related to driver's licenses and lives with and without cars. While they exchanged information about how to get a driver's license in their countries, they also expressed their values and perspectives about owning a car. Many silences were observed during this session: two 4-second silences, one 5, 6, 8-second silences, two 7-second silence, one 13-second silence, and one very long 20-second silence.

Jennifer and Yoko - Session 5 at Individual Room. After checking if they could hear each other, there was 8-second silence. Yoko broke the silence with a question "what are you going to do during the winter vacation?" After the sequence, there was another 5-second silence. Yoko again broke the silence by asking if there was anything that Jennifer wanted ask her about Japan. Instead of answering the question, Jennifer asked Yoko if there were any Japanese culture or customs that she wanted to tell her. After Yoko answered the question, Jennifer asked Yoko 
where the best places to visit in Japan were. Yoko listed several places and explained the reasons why she thought these places were good places to visit. After that, there was another long silence, which lasted for 10 seconds. Jennifer broke the silence with asking if there was anywhere that Yoko wanted to visit in the United States. Yoko listed places while Jennifer listed several places she recommended. The sequence eventually ended followed by 9 -second silence. Jennifer broke the silence by asking Yoko if there was anything else that she wanted to know about the United States. The topic then shifted to Akihabara, a city in Tokyo.

There were four big topic changes during the sessions. Although most of the things that they talked about was general information, there were several parts where both Yoko and Jennifer expressed their values and perspectives, such as living cost and safety in big cities. The conversation patterns remained a question-and-answer format and there was no conversation based on information learned from the previous sessions.

There were ten silences during the session: one 4-second, 6-second, 7-second, 8-second, 9-second, 10-second silences, three 5-second silence, and a very long 19-second silence.

Mary and Yoko - Sessionl at the Café. Since this was the first session for them, the main topic of the session was self-introduction. They exchanged basic information, such as academic year, academic major, hobbies, plans for the summer and after the graduation, and foreign languages they had learned. There was one communication breakdown during the session. While Mary was trying to explain her family members, she could not express what she wanted to say as below.

\section{Excerpt 1:}

101 Yoko: Chuugokutte mada hitorikko nandeshitakke (Does China still have "one-child policy"?) 
102 Mary: Demo ima, aa ....aa...aa .....chichi mo haha mo hitorikko, aa.... de....uun...kodomo wa kodomo wa.....

(But now, ummm, both father and mother are only children, and ummm their child, their child is... )

103 Yoko: Hai.

(Yes.)

104 Mary: Futari, futari, aaa futari, aa...

(Two, two children ummm...)

105 Yoko: Hahaha

106 Mary: Hanasemasen.

(I can't talk. )

107 Yoko: A, ieie.

(No, that's O.K.)

108 Mary: Yes.

(Hai)

109 Yoko: Yukkuride, zenzen

(You can take your time.)

In spite of her partner's encouragement, Mary gave up the explanation. The conversation sequence was mainly one of them asking a question and the other answering. There were three silences: two 7-second silences and one 12-second silence.

Marry and Yoko - Session2 at the Café. The session started with them asking each other's recent events. They talked about current weather and preference on seasons. While Yoko was telling her recent events, the topic was shifted to driver's licenses, which triggered a fairly 
long sequence about getting a driver's licenses in both countries and lives without cars. Then the topic went back to the recent event and connected to the topic about the preference of the ice cream flavors. That sequence triggered another sequence about Taro, which Yoko was unfamiliar with. Topics during the session were cohesive and showed natural transitions between topics. There were two silences during the session: one 8-second and one 6-second.

Mary and Yoko - Session 3 at the Café. The conversation started from Mary asking Yoko what she was going to do after the session. Yoko talked about her part time job, which was what she was going to do after the session, and then Mary talked about her part time job, too. After the sequence, the topic changed to their classes, such as how many hours a week they had classes, and then shifted to Mary's Japanese class. After that, the conversation continued to the experiences of studying other foreign languages. After 12 seconds of silence, Yoko asked what Mary did last weekend. As Mary was telling Yoko what she did past few days, she also told that Thanksgiving break was coming soon. Yoko probably thought Thanksgiving was a religious holiday and asked Mary if she was a Christian. They talked about common religions in their countries; however, they did not talk much about their own religious beliefs. The conversation patterns remained mainly a question-and-answer format. There were six silences during the session: one 6-second, 7-second, 8-second silences, two 9-second silences, and one long silence, which lasted for 12 seconds.

Mary and Yoko - Session 4 at the Café. There was a serious sound problem at the beginning of this session. It seemed like only Mary's end had a problem and there was no problem at Yoko's end. It took about 20 minutes before they finally started the conversation; however, the session had to be ended due to another sound problem. The length of the session ended up with 10 minutes and 28 seconds, which was only a half of the usual session. 
The conversation started with asking each other recent events. Mary was talking about her Thanksgiving break while Yoko was telling Mary that she finally got her driver's license. Then, Mary talked about her plan for the winter break, which dominated this short session. The content of the conversation include both general and personal matters. Although it is hard to judge if a silence was due to a sound problem or not, according to Mary, there were two silences during the session: one 9-second silence and one 6-second silence during the session.

Mary and Yoko - Session 5 at the Café. The conversation started by Mary telling Yoko this was the final week of the semester. They asked each other about winter break and when winter break starts, then the topic shifted to the summer vacation. After Mary told Yoko that her family members live in different places, Yoko asked Mary if she gets homesick, which let Mary express her feelings. The topic switched to roommates. They shared their experiences that they had had with their roommates. While Yoko was explaining her experiences of both having a roommate and living by herself, she also expressed her feelings of loneliness of living by herself. After a very long silence, which lasted for 29 seconds with Mary's "hmmm” at 17 seconds after the silence began, Mary broke the silence by asking what Yoko was going to do that week with laughing wryly. It was clear that Mary asked the question to end the silence. While Yoko was telling Mary that she and her friends were going to celebrate her friend for passing a certification exam, she explained how hard it is to pass the exam and showed praise and respect to her friend for an extended time period. The expected response for her comments would be "That's great!" or any praise to her friend; however, Mary simply responded, "I see (Soo desuka)," although it was not clear if Mary fully understood what Yoko was explaining. There was another long silence. The total length of the silence was 15 seconds. Mary attempted to break the silence and say something at the 5 and 11-second mark. After the silence, Mary managed to come up with a 
question to ask what Yoko does other than study. Almost all of the conversation patters were a question-and-answer format and topics jumped twice without strong connections between topics during the session. Besides these silences mentioned above, there was one 5 and 6-second silences, and three 9-second silences during the session.

Jennifer and Keiko - Session 1 at the Café. At first, they introduced themselves and exchanged basic information, such as academic year, academic major, plans after graduation, hobbies, things that they did during the break, and travel experiences. There was a relatively long sequence of conversation when they talked about experiences of climbing Mount Fuji because both of them had. There were five silences: one 5-second, 6-second, 9-second, 11-second, and 15-second silences although two of them, 9-second one and 11-second one, were made when Keiko was using a dictionary for Japanese words that Jennifer could not figure out their meanings.

Jennifer and Keiko - Session 2 at the Café. The conversation started with asking about what they had done last weekend. While answering the question, Keiko talked about her plan to go to Thailand that weekend and the sequence lasted for quite a long time. The topic shifted to Keiko talking about another planned trip to European countries. After the segment, the topic shifted to vacations in general and then Christmas vacation and things they do during the Christmas season. There were two silences: one 8-second and one 5-second. The conversation pattern was still a question-and-answer format; however, each sequence became longer and transitions of the topics were smoother.

Jennifer and Keiko -Session 3 at the Café. The conversation stared from Keiko checking if Jennifer was going to be on the break, which was mentioned in the previous sessions. Then the conversation continued to plans for the following week followed by Keiko's story about her trip 
to Thailand. After that, the topic shifted to current weather and snow related matters. Keiko introduced a new topic, Christmas. Jennifer shared things that she usually does during Christmas and the topic shifted to their plans for Christmas break. The topic shifted back to Christmas again and they explained what people usually do during Christmas and New Year's Day. Keiko then shared what she usually does on New Year's Day. The topics of the conversation were limited and they shared their views on their cultural customs as well as personal experiences much deeper than previous sessions. There were seven silences during the session: two 4-second silences, two 5-second silences, two 6-second silences, and one 8-second silence.

Jennifer and Keiko - Session 4 at the Café. Keiko started the conversation by telling current weather conditions and that she had caught a cold. After that, she was talking about her trip to Akita, a city in Japan, and what she did during the stay. The topic naturally shifted to barbecue and they discussed this with more details, such as common ingredients and places they have barbecues. Then Jennifer asked Keiko what Japanese people do outside during winter. Keiko used a term "irumineeshon (illumination)," meaning, "Christmas lights," while she was answering the question. However, there was a misunderstanding between them. The sequence is shown below

Excerpt 2:

233 Keiko: Ato wa, ironnatokoro de irumineeshon ga yatteiru node. (There are many places decorated with “irumineeshon")

234 Jennifer: Hee

(Oh really?)

235 Keiko: irumineeshon tte ookii kaijoo toka (You can find "irumineeshon" in big exhibition area...) 
236 Jennifer: Hai Hai

(Uh-huh)

237 Keioko: Chiisai kaijoo, anoo chiisai irumineeshon dattari, moo honto ni ironna tkoro de takusan yattemasu.

(...and small area. You can find it everywhere.)

238 Jennifer: Aa, soo desuka.

(Oh. I see.)

239 Keiko: Soo, sore o minna fuyu wa mini ittari.

(Yes. So during the winter, we go to see them.)

240 Jennifer: Aaa.. Iidesune!

( That sounds great!)

241 Keiko: Soo. Demo amerika dato kakuie de irumineeshon ga sugoi yone?

(Yes. But don't houses in the United States have lots of “irumineeshon?”)

242 Jennifer: Aa hai.

(Ah, yes.)

243 Keiko: Iegoto ni irumineeshon, yatteru?

(Does each house have “irumineeshon?”)

244 Jenifer: Anoo. Sumimasen, irumineeshon tte nandesuka?

(Ah, I'm sorry, what is “irumineeshon?”)

In this sequence, it is not clear if Jennifer misunderstood what "irumineeshon" was or if she pretended to know it. In any case, it was a quite long sequence for not knowing what the topic was. Overall, transitions between topics were very smooth and no breaks between them. There was only one silence, which was 7 seconds, during the session. 
Jennifer and Keiko - Session 5 at the Café. The conversation started from Jennifer referring Keiko's trip to Europe, which was mentioned in the previous sessions. Then the topic shifted to weather, mainly about snow, and Keiko asked Jennifer if she was going home this week, which was also mentioned in the previous session. Jennifer talked more about her plans during the winter break and Keiko mentioned her thesis and that she had to work on during the winter break. The topic shifted to her thesis topic, food and culture, and Jennifer expressed that she also had a strong interest in food, especially Japanese food. She also talked about her dietary life when she was in China. The topic shifted to cafeteria, Halloween and Christmas customs. These topics seemed unrelated; however, they were loosely connected and the transition was very smooth. Most of the time, Jennifer or Keiko shifted the topic voluntarily by telling their stories instead of asking their partner's questions, which was common practice during the first sets of sessions. There were four silences during the session: One 4-second silence, two 6-second silences, and one 8-second silences.

Marry and Keiko - Session 1 at Individual Room. At the beginning of the session, there was a technical problem with sound for 10 minutes. After the problem was solved, they exchanged basic information such as academic year, hometown, academic majors, upbringings, hobbies, plans after graduation, places they want to go, and foreign languages they know and they want to learn. There were two silences: one 6-second silence and one 10-second silence. Mary and Keiko - Session 2 at Individual Room. Mary started the conversation with asking what Keiko was going to do that day. However, Mary seemed to misunderstand what Keiko said.

Excerpt 3: 
11 Mary: [Keiko] san wa kyoo wa nani o surutsumori desuka?

(What are you going to do today, [Keiko]?)

12 Keiko: Eeetto, kyoo wa kono ato, Tokyo sukaitsurii, wakarimasuka?

(Well, after this, I..... Do you know what "Tokyo Skytree" is?)

13 Mary: Soodesuka

(I see.)

14 Keiko: Sukaitsurii no chikaku no machi ni itte, uchiaase o shimasu.

(I'm going to go to the town near "Tokyo Skytree" and have a meeting for business.)

15 Mary: Soo desuka. Omoshirosoo desune.

(O.K. That sounds fun.)

In line 12, while Keiko was telling her plan, she asked if Mary knew what "Tokyo Skytree" was. The expected answer would be "yes" or "no;" however, Mary misunderstood Keiko's question and answered "I see."

After that, Mary told Keiko what she did that day. Then the topic shifted to her Japanese class and Mary's experiences with studying foreign languages. During the sequence, there was another misunderstanding.

Excerpt 4:

$84 \quad$ Keiko: Ryuugakusei toka inain desuka?

(Are there foreign students (at your school)?)

85 Mary: Eeetto, watashi wa uun, nihongo no ryuugakusei ni naritain dakedo, uun, watashi wa uun, gakkoo no toki ni jikan ga nainode, naiga, na..uuun, ga arimasen, naishi, natsu mo jikan ga naishi, watashi wa tabun sotsujyoo ato de, uuun, 
sotsujyoo ato de nihongo o, no ryuugakusei ni natsu, narutsumoridesu.

(Well, I want to study abroad but I can't because I have no time during school. So maybe after I graduate I'm going to study abroad.)

86 Keiko: Purinsuton daigaku ni nihonjin wa ryuugaku shitenaindesuka. (Are there students from Japan at Princeton?)

87 Mary: Uuun, shirimasen

(Well, I don't know.)

In this sequence, Mary misunderstood Keiko’s question as “Do you want to be a foreign students (do you want to study abroad)?” and gave her a long answer, Keiko did not stop Mary's answer and restated the question when Mary's answer was over.

Keiko then asked if Mary watches Japanese anime then the topic shifted to the vacations in both countries. The topics were, however, rather general, such as when the vacations start, and not much personal information involved in the sequence. Conversation patterns were mainly a question-and-answer format and most of the contents of the conversation remained general rather than personal. There was only one silence during the session, which lasted for 9 seconds.

Mary and Keiko - Session 3 at Individual Room. In the past sessions, the conversation patterns have been a question-and-answer format; however, this session started from Keiko talking about her trip to Thailand and afterwards, Mary started talking about her plan during Thanksgiving break. Their sequences, which started from telling stories without being asked, were quite different from the previous sessions. Since Thanksgiving was unfamiliar to Keiko, Mary explained what she usually does on Thanksgiving Day. Then the topic shifted to other holiday events, such as Christmas and New Year's Day. They were talking about rather general 
customs in their country instead of talking about what they do or did. There were only two silences during the session: one 8-second silence and one 7-second silence.

Mary and Keiko - Session 4 at Individual Room. At the beginning of the session, Keiko was trying to ask Mary about Thanksgiving break; however, Mary thought she was asking about winter break even though they were eventually on the same page. As a part of the sequence about what Mary did during the break, Mary explained about "NaNoWriMo (National Novel Writing Month)." Probably because Keiko had never heard of it, she only gave minimum responses to it. Keiko also told Mary about her trip to Akita. This topic was shifted to places where Mary wants to visit and then her plan for summer. After that, they talked about graduation seasons in both countries, and then Mary told Keiko about her internship experience that she had last summer. While they were talking about the company that Mary worked as an intern, there was a conversation broke down as below.

\section{Excerpt 5:}

144 Keiko: Sono intaan shippu?

(That internship?)

145 Mary: Hai

(Yes.)

146 Keiko: Hataraku no wa jibun kara yarasete kudasaitte onegaishini ikun desuka. (Do you go to the company and ask them to give you an internship?)

147 Mary: Uun...

(Hmmm.)

148 Keiko: Daugaku ni boshuu ga aru?

(or do they come to the university and recruit (boshuu) students?) 
149 Mary: Digaku no booshi?

(University's hat (booshi)?)

150 Keiko: Boshuu, daigku ni iishito imasen katte itte senden ga kuru?

(Recruit (boshuu), do they come to the university and ask if there are any good candidates?)

151 Mary: Hai.

(Yes.)

152 Keiko: Sore de yarimasu tte itte yaru?

(And you said "yes" and started working?)

153 Mary: Uuun, chotto wakarimasen

(Hmmm. I don't understand.)

154 Keiko: Muzukashii ka [Laugh]

( It's difficult, isn't is? [Laugh])

155 Mary: Hai, muzukashii [laugh]

(yes, it's difficult.)

156 Keiko: Ja daijoob desu, [Laugh] gomen ne [Laugh]

(That's O.K., then, [Laugh] I'm sorry. [Laugh])

As it shows above, even though Keiko tried to explain the word "boshuu," she gave up with explaining after Mary said, "I don’t understand.” After that, Mary explained about internships in general in her university. She then asked Keiko about her academic specialization. Keiko explained about her specialization as well as her thesis topic. The thesis topic was about job-hunting; therefore, the topic shifted back to internships. Mary explained more about her experience as an intern in both university and high school. Although the transitions between 
topics were natural, the conversation patterns remained mainly a question-and-answer format. During this session, there were two silences: one 8-second silence and one 5-second silence. Mary and Keiko - Session 5 at Individual Room. The conversation started from Mary telling Keiko that this was the last week of the semester and she had to write a paper before the break. Then Keiko also told Yoko that she also had to write a paper. After the sequence, Keiko talked about her trip to Disneyland last weekend and the topic shifted to amusement parks in general. While Mary was talking about her experience with an amusement park, she mentioned that she went there to play violin. The topic then naturally shifted to music afterword. They talked about musical instruments that they had played and concerts that they had been to. After the sequence, Mary asked Keiko what she does other than study. They talked about their part time jobs and the topic shifted to future plans after graduation. Although there were some question-and-answer sequences, there were some sequences started with one of them telling stories without being asked. There were varieties of topics during the session; however, they were some topics that they had already talked about in the previous sessions, such as plans after graduation. There were four silences during the session: two 6-second silence, one 7-second silence, and one 10-second silence.

Self-disclosure. Next, in order to examine how the emotional connections with their partners were expressed in the conversations, the analysis of self-disclosure was conducted. The results were organized by each participant and displayed in Appendix M-T for visual analysis. The data include the number of total AS-units during the session by each participant, the number of AS-unit of level one self-disclosure, and the number of AS-unit of level two self-disclosure. During the sessions, there was no level three self-disclosure observed. That is quite 
understandable because the emergence of level three self-disclosure requires a firm, close relationship.

\section{Integrative analysis for each participant}

In this section, the results shown and described above will be combined and examined from each participant to answer all of the research questions. Each question item on the line graphs have been shortened throughout the remainder of the paper. Below is a key showing the original question and its shortened version.

Table 3.

Original question and its shortened version

\section{Original survey question}

Getting to know my partner in this environment gave me a sense of connection with my partner.

I was able to form distinct impressions of my partner through the online medium in this environment.

This form of online communication in this environment is an excellent medium for social interaction.

I felt comfortable interacting with my partner in this environment.

I felt comfortable disagreeing with my partner in this environment while still maintaining sense of trust.

I felt that my point of view was acknowledged by my partner in this environment.

Online discussions in this environment help me to develop a sense of collaboration.

\section{Shortened version}

Sense of connection

Distinct impressions of partner

Approval of medium

Comfort level of interaction

Comfort level of disagreement Acknowledgement of point of view Sense of collaboration

Jennifer. Session 1:Café. The graph in Appendix E shows that she rated higher on "sense of connection," "distinct impressions of partner," and "approval of medium" for the session in 
the café than the session in the individual room. She compared the first experiences in both environments and stated that seeing the avatar of her partner gave her "a better sense of connection" than just talking to her partner in the individual room. She also described the experience as it felt more like "an actual face-to-face conversation" at the café environment while talking in the individual room was more like "having a phone conversation with someone." Even though she mentioned the interaction in the café environment was like "an actual face-toface conversation," she pointed out that she had difficulty in conveying some of her ideas when she was not sure how to say something in Japanese because her partner could not see her gestures. Although the conversation pattern was strictly a question-and-answer format, there were a quite a few numbers of self-disclosures observed during the session. Most of her selfdisclosures were provided by adding more personal information after answering for her partner's question.

Individual Room. In the individual room environment, the scores for "comfort level of interaction" and "acknowledgement of point of view" were higher than the ones in the café. While the score was high for "acknowledgement of point of view," the score for "sense of connection" was very low. After the session, she commented that "overall I did not feel nervous about speaking with a stranger in a language that I have only a rudimentary knowledge of.” This may be a part of the reason why she rated higher for "comfort level of interaction" in this environment. However, she also pointed out the difficulty in knowing when her partner had finished speaking; therefore, she sometimes interrupted her partner accidentally. Compared to her partner in the café environment, her partner in this environment had a tendency to have longer responses and use inversion, which is common speech style in Japanese. The followings are examples of inversions made by her partner, Yoko. 


\section{Excerpt 5:}

69 Yoko: Kookoo-no sannenkan-wa zutto hokkaidoo-ni imashita.

(High school) (for 3 years) (for quite a while) (in Hokkaidoo) (stayed)

Hokkaidoo-no kookoo-ni nyuugaku shitanode

(Hokaidoo’s) (high school) (entered) (because)

Before inversion: Hokkaidoo-no kookoo-ni nyuugaku shitanode, kookoo-no sannenkanwa zutto hokkaidoo-ni imashita.

71 Yoko: Moo hontoo-ni iitokoro deshita-yo. Tabemono-ga oishikute, (Very) (good place) (was) (food) (was delicious) (because)

Before inversion: Tabemono-ga oishikute, moo hontoo-ni iitokoro deshita-yo.

Considering using inversions is most likely due to the personal preference, Jennifer's impression, the difficulty in knowing when her partner had finished speaking, is probably came from Yoko's speech style not much from the environment.

Session 2: Café. The graphs in Appendix E show drops on "approval of medium” and "sense of collaboration" and no improvement in the café environment. However, all of her comments after the second sessions were positive. She mentioned that she felt more connected with her partner because they learned more about each other. She also pointed out that she shared with her partner some of her interests and said she "felt more comfortable" sharing about her "likes and typical holiday customs." One of the main topics in this session was her partner, Keiko's plan of trip to European counties. While Jennifer asking Keiko about various European counties, she expressed her interests in these places. As a result, there were relatively more level two self-disclosures than level one self-disclosures in Jennifer's results. These common interests also was a part of the reason why she "felt more comfortable." She also mentioned that she felt 
more like "speaking with a friend than meeting someone new for the first time." Although she did not specify why she did nor what made her feel that way, visibility of her partner though avatar might be the reason.

Individual room. On the other hand, the scores from the session in the individual room environment show significant improvement on "sense of connection" and some improvement on “distinct impressions of partner" and "comfort level of disagreement." However, the scores on "comfort level of interaction," "acknowledgement of point of view" and "sense of collaboration" show drops. She mentioned that she had "more material to form an impression" of her partner and that matches the improvement on "distinct impressions of partner." As mentioned above, the main topics were Disney movies and Disneyland, which both Jennifer and Yoko like. It seemed very easy for both of them to express their preferences and feelings while they were talking about these topics. This resulted in higher numbers of level two self-disclosures for both of them and furthermore, Yoko's higher self-disclosure may be a major contribution for improvement on "distinct impressions of partner."

Session 3: Café. Although only two areas, which were the scores for "approval of medium" and "comfort level of interaction," show improvements, all of the comments from the session in the café environment were positive. Jennifer felt like she shared more with Keiko and was more comfortable with sharing with Keiko some of her customs during the holiday. She also felt that Keiko was interested in what she was sharing. This matches the score increase in “comfortable with interacting." The graph in Appendix M shows that during this session, Jennifer had the most AS-units among all sessions. She also had higher both level one and level two self-disclosures. This is probably because Keiko kept asking her questions. There are many sequences have this pattern during this session: 1. Keiko asks Jennifer. 2. Jennifer gives initial 
answer, which was not included in self-disclosure. 3. Jennifer keeps adding information to the initial response or express her feeling to the initial response, which were determined as selfdisclosures. Therefore, the graph in Appendix M for Keiko shows while she high AS- Therefore, the graph in Appendix M for Keiko shows while she had high AS-units, the numbers of selfdisclosure was relatively low. This conversation pattern might make Jennifer feel Keiko was interested in what she was sharing.

Individual room. The scores on the graph in Appendix E remain the same except for an improvement on "sense of collaboration." She looked back on the session and said feeling an emotional connection with her partner was about the same as the last time; however, she found "more stalls in the conversation" during this session. As mentioned above, there were seven silences during the session, which includes very long 20-second silence. This silence happened right before the end of the session. When time was up, the researcher sent messages to both of them and that broke 20 -second silence. Therefore, this could have been longer silence if there were more time left. This long silence obviously shows there was no topic that both of them wanted to talk about; however, it may also show that they felt less pressure to break the silence.

Session 4: Café. The graph in Appendix E shows that the scores on "distinct impressions of partner," "approval of medium," "comfort level of disagreement," "acknowledgement of point of view," and "sense of collaboration" show improvement. As a result, the average score also clearly increased. This is supported by the fact that her comments after the session were all positive. She stated that she felt that she connected with her partner better each time and noticed that the amount of "gaps" in their conversation also decreased every time. She analyzed the reason and she thought that that was probably because they started their conversation with topics that they both were familiar with and were interested in. The main topic of this session was food 
for grilling. Considering that Jennifer had interests in food in general, which was mentioned in the previous session, the topic contributed to cohesive and smooth conversions as well as improvements on the scores as mentioned above.

Individual room. In the individual room environment, even though all of the scores except for "sense of collaboration," which shows a drop, remains the same, the comments after the session have a negative tone. She felt the session was "a little less connected between us" and noticed "there were more breaks between our conversations than usual." Indeed, as mentioned above, there were nine silences including 13-second one and 20 second one. She suspected that the topic they chose to guide their conversation was the cause of the breaks; however, she also admitted that she "did not feel quite comfortable to change the topic to something else" when the chance to do so arose in their conversation. This comment explains why three-fourths of the session was dominated by topics related to driver's licenses and lives with and without cars during this session.

Session 5: Café. Although the scores in the café environment show no improvement as the graph in Appendix E shows, her comments were all positive just like the previous session. She looked back at the session and mentioned, "Compared to earlier sessions, I definitely think that I have a more emotional connection with the other party." This emotional connection might help her to produce higher numbers of both level one and level two self-disclosures.

She also reviewed their relationship and mentioned that she and her partner "have moved from two new strangers to close acquaintances" through the sessions and the café environment has "facilitated the process." She compared the two environments and commented as below. 
Being able to see each other's avatars has given me a sense of actually speaking with her about a variety of topics whereas speaking to each other in individual room environment felt less so.

Combining this comment and the comment that she made after the fourth session in the individual room, bringing up various topics seems to require a positive attitude towards their relationship and a feeling of comfort while talking to her partner.

Individual room. There was only one improvement within the scores on the graph in Appendix E, which was for "approval of medium." Her comments show the stale state of the relationship. She mentioned that she "did not feel a more or less emotional connection than previous sessions." She looked back on the previous session and mentioned that she and her partner "did connect more" compared to the earlier sessions; however, she felt that the level of emotional connection "plateaued" once they reached a certain level. Even though she admitted that she still learned new things about her partner, she did not feel that they allowed her to "form a deeper connection" with her partner. The content of the conversations clearly show a “plateaued" emotional connection. Instead of asking or telling about recent events or talking about their plans, they started asking each other what their partners wanted to know about their cultures.

Mary. Session 1: Café. The graphs in Appendix F show that she rated "sense of connection," "distinct impressions of partner," and "approval of medium" higher for the session in the café environment while rated "comfort level of interaction" higher for the session in the individual room environment. She rated most of the areas relatively high after the first session compared to the other participants. 
These ratings are backed by her comments. In the café environments, she felt "quite closely connected" to her partner during the session; however, she "struggled over when it would be appropriate to jump into the conversation" and she noticed that often times there were "longer awkward pauses" than she felt there would be in a face-to face conversation." She also felt "the urge to gesture" during the session even though she knew her partner could not see her. The communication breakdown, which was mentioned above, might be due to the nervousness and that experience probably made her nervous even more as she mentioned pauses and mistakes in the conversation made her feel "a great deal" more in this environment than in the individual room environment.

Individual room. As mentioned above, "comfort level of interaction" was rated higher in the individual room environment; however, "sense of connection," "approval of medium," and “distinct impressions of partner" were rated lower. She mentioned that she felt "slightly more comfortable in this session" than the session in the café environment. She described herself in the session as "a little less engaged in the conversation" because she did not have "the overwhelming urge to gesture" at her partner unlike she did in the café environment. In addition to that, she noticed that even though there were more pauses in between topics, she felt less uncomfortable than she did during the session in the café environment. As a result, she felt that the conversation seemed to "flow more naturally" when she could not "see" her partner.

Session 2: Café. In the second session, as the graphs in Appendix F show, the scores for "sense of connection" and "approval of medium" dropped while the scores for "distinct impressions of partner" and "comfort level of interaction" were improved in the café environment. She mentioned that she felt she and her partner had "a slightly better emotional connection" since she felt that both she and her partner responded more to each other. She 
admitted that the pauses were still "somewhat awkward," and sometimes she felt "a bit unsure as to how to respond" to her partner because she was still unfamiliar with her partner. However, she also felt that the topics that they discussed were "slightly more personal (driving school, ice cream) and more focused than last time." The graph in Appendix S shows that both Mary's and Yoko's AS-units in this session were the fewest among all 20-minute sessions. This is because Mary used a great amount of fillers when she was trying make herself understood; however, all of the fillers were omitted when the original transcripts were refined into the one with AS-units.

Individual room. In the individual room environment, the graphs in Appendix F show that the score for "distinct impressions of partner" improved; however, the one for "sense of collaboration" dropped. She felt that she had probably the same amount of emotional connection as she did last time. She mentioned that the topics that they discussed were "about as personal as they were last time," and they were "strictly cultural, rather than personal." Just like the first session, she still felt quite comfortable continuing the conversation in this environment, and even the few longer pauses still "didn't feel particularly awkward or out of place." Lastly, she compared her partners in both environments and felt like her partner in the individual room environment said less than her partner in the café environment did.

Session 3: Café. In the café environment, the graphs in Appendix F show that there are not many changes except for the improvement on "sense of connection" and drop on "distinct impressions of partner;" however, her comments show the significant improvement in her comfort level. She mentioned that she felt "a lot more comfortable" with her partner this time around. As a result, she looked back on their conversation and thought even though they talked "a bit more about more serious topics like religion and work," she felt "less awkward" than she did last time when they talked about "more silly, more personal topics." She also appreciated her 
partner's effort and mentioned that her partner was also "more open and jumped into the conversation more;" therefore, the pauses were "less awkward and occurred less often." This may be the reason why the graph in Appendix $\mathrm{N}$ shows high numbers of AS-units and selfdisclosures, both level one and level two. Most of the self-disclosures took place as additional information, which explains her initial response. At the same time, she also felt that she "interrupted her partner a lot" and thought time lag that Second Life has was the reason.

Individual Room. Despite the fact that there is no change in scores on all of the graphs in Appendix F, the comments in the individual room environment turned out to be generally negative. She felt "there was slightly less of an emotional connection than last time." As a reason, she adduced two factors: the topic that they discussed was too difficult for her to explain in Japanese, and the fact that she received less of a response from her partner than she did in the last few sessions. She also felt that she had to "jump in a lot more to continue the conversation" because she felt that both of their responses were shorter than they were before. The graph in Appendix $\mathrm{N}$ shows significant lower self-disclosures, both level one and two, during the session. The content of the conversation was very general and the majority of the conversations ended up with explanations of cultural matters. Her partner, Keiko's self-disclosure was even lower than Mary as the graph in Appendix T shows. This shows that they simply exchanged the facts rather than their views or feelings during this session.

Session 4: Café. In the café environment, there was a technical problem and the session lasted only for 9 minutes, which was less than a half of the normal session length. This naturally results in fewer AS-units and self-disclosure as the graph in Appendix S shows. This may be a part of the reason why the scores for "approval of medium," "comfort level of disagreement," and "sense of collaboration" on the graph in Appendix F drops. Despite these drops, her 
comments were overall positive. She felt that they managed to have a "nice conversation" in what little time they had. She also felt "very comfortable" speaking with her partner, and she had the impression that they "responded well to each other's questions." Even when they had two very long pauses, she felt that they were "relatively non-awkward," and she felt like she and her partner "took turns redirecting the conversation from the pauses."

Individual room. In the individual room environment, the scores for "sense of connection" and "sense of collaboration" on the graph in Appendix F show improvement and her comments for the session were also generally positive. She felt "extremely comfortable" during the session. Even though she admitted that it took some time to get settled, and she "struggled a bit" to figure out how to say what she wanted to say, she felt that the conversation "overall progressed very smoothly." She appreciated her partner's effort to take the time to wait for her to finish what she wanted to say and she felt that her partner paid attention to what she said; therefore, she felt like what she was saying was "being acknowledged." She also appreciated her partner's effort to maintain a coherent discussion by jumping into the conversation, even though it was a little difficult for her to fully understand some of the things her partner said. One of the examples would be the conversation broke down, which was shown in Excerpt 5. These generally positive impressions might trigger higher numbers of self-disclosures as the graph in Appendix T shows. After giving an initial response, she tended to add more information or develop a new topic based on that topic during this session. However, as Keiko's numbers of self-disclosures shows, this is rather one-way self-disclosure rather than two-way self-disclosure, telling about themselves to each other.

Session 5: Café. In the café environment, there are some improvements and drops on the scores on the graph in Appendix F. In particular, scores for "distinct impressions of partner," 
"approval of medium," "comfort level of disagreement" improved while scores for "sense of connection" and "comfort level of interaction" dropped. The comments for the environments were, however, overall negative. During the session, she felt difficulty in finding things to say to her partner; therefore, it was difficult for her to be emotionally connected to her partner. She felt like she had to "constantly jump in to fill in the pauses," which were "pretty long and often." Although she managed to "steer the conversation into some new topics" that they haven't covered before," she strongly felt that both of them gave "really short and somewhat awkward sentences." Her efforts to continue the conversation was easily observed as above. Interestingly, the graph in Appendix S shows the highest numbers of level one self-disclosures among all of the sessions. Looking at the interactions between them, it was clearly because she was trying to fill the conversations with her "facts" to keep the conversation flowing.

Individual room. The scores for "distinct impressions of partner," "approval of medium" and "comfort level of disagreement" on the graph in Appendix F improved. However, the scores for "sense of connection" and "comfort level of interaction" dropped. The comments for the session were generally positive. She felt "quite comfortable" with her partner during the session and she even thought this session was "the most comfortable" she felt with her partner among all of the sessions. These comments, however, conflict with the dropped score on "comfort level of interaction." She described the session as the session had "coherent conversation" with "only a few short pauses," and she felt she and her partner were able to cover "a wide variety of new topics." Continuing from the previous session in the environment, she felt that both she and her partner "participated strongly in the conversation, jumping in to maintain the flow of the conversation." Despite of the fact that she had a hard time hearing her partner at times and had to ask her partner to repeat, she felt "extremely comfortable" talking to her partner. The graph in 
Appendix $\mathrm{N}$ shows high numbers of self-disclosures especially in level one. It looks similar to the one she had in the café environment; however, conversation patterns in two environments were very different. While she was adding more information to her own answer to make her answer longer in the café environment, in the individual room, she had more questions from her partner, Keiko, and as a result, the number of initial answers as well as the number of additional information were higher. Therefore, even though the numbers of the self-disclosure looked similar, the naturalness of the conversation flow was very different compared two environments.

Yoko. Session 1: Café. The graphs in Appendix G show that she rated higher for five out of seven areas in the café environment than the individual room environment, which were “distinct impressions of partner," " approval of medium," "comfort level of interaction," “comfort level of disagreement," and "sense of collaboration." She looked back on her session and said that she was very nervous and she was attentive to her selection of words and the speech style because she did not know how much her partner would understand her Japanese. Looking at their interactions, she asked more questions to her partner than telling about herself to her partner during this session. In spite of the nervousness, she still thought seeing her partner's avatar and sharing the environment lowered the sense of insecurity that was caused by communicating with a stranger who was from a different county.

Individual room. She rated most of the areas fairly low in this environment as the graphs in Appendix G show. Her comments backed these lower ratings. She mentioned that although she was relieved when she learned that her partner's language proficiency was relatively high, she felt "restless" and felt like she was talking to herself because sometimes her partner did not give "supportive responses (aizuchi)" to her utterance. In addition to that, when the conversation was interrupted, she felt very uncomfortable because she felt like both of her and her partner 
were having trouble breaking the silence. During the session, she asked more frequent and rather short questions to her partner, Jennifer. When Jennifer asked her questions, the answers tended to be short and simple. The graph in Appendix Q shows the tendency: higher AS-units and relatively lower self-disclosures.

Session 2: Café. As the graphs in Appendix G show, there are four areas that show the improvement on the scores on the graphs: "sense of connection," "comfort level of interaction," "acknowledgement of point of view," and "sense of collaboration" in the café environment. She found the session "interesting" because they were able to talk about topics that were more familiar and learned that they shared basic values, such as inconvenience of not having a supermarket nearby. She also noticed that seeing her partner's avatar might help her feel like she had met her partner before. The AS-units on the graph in Appendix S were relatively low for the same reason why her partner's number of AS-units was low: due to the fillers that her partner made.

Individual room. The graphs in Appendix G show improvement on six out of seven areas in the individual room environment. Especially the score for "distinct impressions of partner" shows a significant improvement. She admitted that she felt difficulty with coming up with a topic at first. However, she and her partner found a common interest, which was Disney movies, and as a result, she felt they became closer than the previous session. The higher number of level two self-disclosures also supports her comments as the graph in Appendix O

Session 3: Café. As the graphs in Appendix G show, there is only one item that shows improvement, which was "comfort level of interaction." However, all of her comments were positive. She felt that she could talk to her partner more comfortably and the topics shifted to more familiar everyday matters. She thought that was because she felt less hesitation to ask 
questions that are more private to her partner than the previous session. As a result, she started feeling that meeting her partner was similar to meeting her friends whom she saw often in everyday life.

Individual room. In the individual room environment, there are two areas that show improvement: "comfort level of interaction" and "comfort level of disagreement." She reported that she felt more comfortable during this session compared to the first and second session because she got to know her partner more with each session. However, since she experienced both environments twice, she noticed the differences between the two environments more clearly and stated that not being able to see her partner's avatar frustrated her sometimes and she felt uneasy in the individual room environment because she felt like she could not settle nor find an object to look at during the session. The graph in Appendix $\mathrm{O}$ shows that she had relatively higher AS-units and level two self-disclosure, the contents of the conversation became more personal and she expressed her feelings, perspectives and values more often during this session. For example, she expressed her feeling about graduation, expectations for the life after graduation, and her view of living in a big city.

Session 4: Café. The graphs in Appendix G show that there is one improved item, which is "comfort level of disagreement," and one dropped item, which is "sense of collaboration" in the café environment. She looked back on the session and said she and her partner could talk comfortably and she started opening up a little more to her partner. She also noticed that she started to feel relieved when she saw her partner's familiar appearance just as if she feels when she finds familiar faces in real life. She even mentioned that it was sad the next session would be the final session. 
Individual room. Although the graphs in Appendix G show no changes except for one drop on "comfort level of interaction," her comments remain negative. She felt more frustration because as she had become closer to her partner, she started having an urged to "meet" her. Even though she felt this closeness to her partner more than ever, she felt the conversation was "temporizing," and she was having difficulty to "seize the psychological distance with her partner." She expressed that it was "just like trying to seize clouds." As mentioned above, the majority of the session was about driver's licenses and lives with or without cars and her partner, Jennifer, did not change the topic because she was not comfortable to do so. It was not clear why Yoko also did not change the topic; however, having difficulty to "seize the psychological distance" may be a part of the reason.

Session 5: Café. The graphs in Appendix G show no differences in the café environment. She mentioned that she was bracing herself and because that was the final session. As a result, she felt like she was not able to provide topics to talk about during the session as much as she wanted. When they had silences, while her partner, Mary, was trying hard to break silences, Yoko was not willing to make an effort to break silences. In spite of the negative points, she still thought it was easy for her to open up to her partner in the café environment partially because they met up before they went to the café and she was able to prepare herself for the conversation.

Individual room. The graphs in Appendix G show improvements in three areas: "sense of connection," "approval of medium," and "comfort level of interaction." However, her overall comments remain the same as the previous session. She mentioned that even though they had an animated conversation in the final session and had built a certain level of closeness each other, she was not able to dispel "unclearness" that she had had toward her partner throughout the 
sessions. This may be a reason why she started the session with a question asking what Jennifer wants to know about Japanese culture, which does not show any accumulated knowledge of Jennifer. She preferred different kinds of communication means or environments, such as Second Life in the café environment or Facebook, if there is another opportunity to interact with people whom she does not know.

Keiko. Session 1: Café. As the graphs in Appendix H show, all of the scores, expect for "comfort level of disagreement" being higher in the café, the scores in the café environment and the individual room were the same. The scores for "sense of connection," "distinct impressions of partner," were relatively low while "approval of medium," "comfort level of interaction," and "sense of collaboration" were relatively high. Although she wished the avatar would have had her partner's actual face, she mentioned that the café environment was "very good to place to interact with her partner.'Examining their interactions, she tended to ask more questions, which is also shown by the relatively high number of AS-unit, and gave minimum answers to her partner, which was shown by the relatively low number of self-disclosures on the graph on Appendix P.

Individual room. As mentioned above, "comfort level of disagreement" was the only area that she rated lower than the other environment. The scores for "sense of connection," "distinct impressions of partner," were relatively low while "approval of medium," "comfort level of interaction," and "sense of collaboration" were relatively high just like the café environment. She mentioned briefly that she experienced some difficulty because she could not see her partner's avatar.

Session 2: Café. There were improvements on the scores for "sense of connection" and "distinct impressions of partner" while there were also drops on the scores for "approval of 
medium" and "comfort level of interaction" on the graphs in Appendix H. Her comments, however, were overall negative. She mentioned that although she talked like the previous session, there were times that she felt an uncomfortable and psychological distance from her partner. She was also not sure what she could talk about with her partner and how deep the topic could go. This comment shows that she was still adjusting the psychological distance of her partner and her.

Individual room. As the graphs in Appendix $\mathrm{H}$ show, there were improvements on the scores for "sense of connection" and "distinct impressions of partner," and "comfort level of disagreement" while there was a drop on the scores for "comfort level of interaction." This drop could be caused by two misunderstandings that they experiences as described in Except 3 and 4. As a result, the scores for "comfort level of interaction" and "comfort level of disagreement" became the same level after the second session. The average of the scores shows a slight improvement. The comments turned out to be all positive. She found it was easier to talk to her partner this time around because she felt "less nervous" compared to the first session. She also felt she had "better mental preparation" this time around.

Session 3: Café. In the café environment, "sense of connection," "comfort level of interaction" and "acknowledgement of point of view" show improvement on the graphs in Appendix H. Her comments were mainly about the contents of the conversation. She mentioned that she understood the differences between Japan and the United States through culturally representative holiday events, such as Christmas and New Year's Day. The interactions with her partner and the graph on Appendix R shows that she still had tendency of asking more questions and give minimum answers to her partner. However, from this session she started asking 
questions, which were based on the information that her partner shared with her in the previous session.

Individual room. There were only drops on the scores on "sense of connection" and "sense of collaboration" and no improvement in this environment on the graphs in Appendix H. As a result, the average of the scores also dropped. However, her comments were positive and strictly about the contents of the conversation. She commented that she discovered new things through the conversation about each other's culture. She also mentioned that the discussions about culture led to a better understanding of each other and as a result, she developed some closeness toward her partner.The graph on Appendix R shows that she had a very limited number of self-disclosures during the session. This was due to the content of the conversation, which was dominated by general customs in their country instead of talking about what they do or did.

Session 4: Café. The graphs in Appendix H show that there were only drops on the scores in the café environment. The score for both "sense of connection" and "acknowledgement of point of view" dropped. As a result, the average of the scores shows some decline. Her comments were also all negative. She realized once again how hard to deliver a message only with voice was. She also recognized the importance of accurate understanding words and their meanings because there are many Japanese words which were adapted from English words yet have different meanings from the original English meanings. She probably mentioned about the misunderstanding about "irumineeshon," which was described in Excerpt 2. Considering their tone of conversation being generally cheerful and supportive, this misunderstanding gave her a negative impact on "sense of connection" and "acknowledgement of point of view."

Individual room. There was only one improvement on the score: the score for "sense of collaboration" on the graphs in Appendix H. Similar to the comments for the session in the café 
environment, she also commented on the difficulty of expressing her opinions to her partner. As described in Excerpt 5, there was an occasion that she was trying to explain something but when she realized that it was hard for her partner, she gave up with explaining instead of trying explaining again. This experience also made her realize the importance of striving to express her opinion even if it may take a long time.

Session 5: Café. The score for "sense of connection" on the graph in Appendix H was the only area that had improved since the previous session and other areas remained the same; therefore, there was only a slight improvement on the average. However, her comments turned out to be all positive. She compared the two environments and felt the ease of conveying the conversation depended on whether or not she could see existence of her partner's avatar. She also commented on the contents of the conversation and said even in the short conversations, it was impressive to know the differences between their cultures as well as on their perceptions. As the graph on Appendix P shows, she kept her tendency of asking more questions and give minimum answers to her partner. However, tone of the conversation was much more cheerful and these questions sounded like came from genuine curiosity to her partner rather than filling the silences.

Individual room. The only area to decline was "comfort level of interaction;" however, it was a significant drop: from 4 to 2 as the graphs on Appendix H show. The average score also slightly dropped accordingly. Her comments were also all negative. She mentioned that she could not sweep away the sense of "discomfort," which she had had since the first session. She also confessed that she tended to forget what she talked about with her partner in this environment because the sessions took place only once a week. This is probably true to her partner Mary. Mary asked Keiko about her plans after graduation in the first session and asked 
her again in this session. There was no sequences that were based on previously acquired knowledge through sessions. Not being able to see the avatar may be a reason for that tendency as Yoko and Keiko mentioned.

4. Further analysis for generalizability. In this section, all of the data presented above will be analyzed collectively to examine if there are any tendencies among all participants, native or non-native speakers.

Similarities among participants. First, similarities among all participants, both native and non-native speakers, will be discussed below.

Visibility of avatar and contents of the conversation. As previously stated with one of the examples in the fifth session, there were several occasions in the individual room environments that participants asked questions, which they had asked in the early sessions, in the later sessions. On the other hand, there was no instance of this overlap in the café environment. Besides this, in the café environment, there were conversational sequences which were based on information that was given in previous sessions. This never happened in the individual room environment. Comments from the participants explain why these things happened. Yoko constantly expressed her uncomfortableness in the individual room and one of the reasons was not having visual cues of her partner. She explained that it was hard to remember what they talked about in the previous sessions without having visual cues. Keiko also pointed out a similar problem. She added since they had sessions only once a week, it was very hard to remember what they had talked about in the individual room environment. Therefore, the visual element of the avatar may help participants remember what they talked about, which is necessary to understand a partner's views, perspectives and values through discussions. 
Time to get to know someone in Second Life. All of the participants expressed general nervousness in the first session even though there were different degrees of nervousness among participants. By the third session, however, all of the participants became relatively conformable with having light conversations about recent events or their own cultures. The actual contents of the conversations support the point. This phenomenon could be a result of adjusting to the restrictions of the media by adapting their language behavior to fulfill the functions of missing non-verbal cues to build interpersonal impressions, as the social information processing perspective of mediated communication explains (Walther, 1992). After the third session, their emotional connections either became stronger or remained static. When there were no common interests or topics that they wanted to talk about, silences happened more frequently in later sessions.

Self-disclosure. Although some level two self-disclosures were observed in the first sessions, they tended to appear more frequently in the later sessions. The contents of the conversation also show the tendency. In the first sessions in both environments, participants exchanged basic information. Hobbies could be considered as level two self-disclosure; however, most of the participants simply told what their hobbies were and the topic switched to a new one, that is, no sequences about hobbies were ever deeply discussed at length. In the later sessions, some participants started to show their values and perspectives voluntarily. Yoko, for example, showed her willingness to make her own money after graduation, and told her partners that she had to compromise her living situation and having a car, in both environments. On the other hand, Keiko hardly ever presented self-disclosures from first session to the last session, in both environments. The reason why the number of her self-disclosure did not increase will be discussed later section. These results lead to the conclusion that the self-disclosures generally 
increase with each session in both environments; however, no particular environment promote self-disclosure.

Silences. Each session had at least one instance of silence; however, longer silences, such as 19 -second silences and 20-second silences, only happened in the session of Jennifer and Yoko in the individual room environment as Table 5 shows. The total seconds of silence of the pair also rose way above any other group. However, the other pair in the individual room environment, Mary and Keiko, did not have very long silences nor a large amount of times being silent.

One possible reason is the conversation patterns of Keiko: she tended to give short answers and ask questions to her partners quickly. Although Mary did not experience longer silence in the individual room, she still noticed that the pauses in the conversation in the individual room "felt less awkward" since there seemed to be "less pressure to continue speaking for a long time." Besides, Jennifer thought that in the café environment, people will be "on their best behavior" because they feel like they are with other party. This is also a possible motivation to avoid longer silences. Therefore, even though a speaker's personality and pair's compatibility can be considered to be a part of the reason, not being able to see the partner's avatars might also be the reason for longer silences. 
Table 4.

Total seconds of silence in each session ( $\mathrm{sec})$

\begin{tabular}{|c|c|c|c|c|c|c|}
\hline Pair & Session 1 & Session 2 & $\underline{\text { Session } 3}$ & Session 4 & $\underline{\text { Session } 5}$ & Total \\
\hline Mary and Yoko (Café) & 26 & 14 & 51 & 6 & 38 & 135 \\
\hline Jennifer and Keiko (Café) & 26 & 13 & 38 & 7 & 29 & 113 \\
\hline Mary and Keiko (IR) & 16 & 9 & 15 & 13 & 29 & 82 \\
\hline Jennifer and Yoko (IR) & 20 & 34 & 66 & 74 & 78 & 272 \\
\hline
\end{tabular}

Table 5 .

Longest seconds of silence in each session ( $\mathrm{sec})$

\begin{tabular}{lrrrrrrr}
\hline Pair & Session 1 & Session 2 & Session 3 & Session 4 & & Session 5 & Average \\
Mary and Yoko (Café) & 12 & 6 & 12 & 6 & 9 & 9 \\
Jennifer and Keiko (Café) & 15 & 8 & 8 & 7 & 10 & 9.6 \\
Mary and Keiko (IR) & 10 & 9 & 8 & 8 & 10 & 9 \\
Jennifer and Yoko (IR) & 14 & 15 & 20 & 20 & 19 & 17.6
\end{tabular}

Distinct impression of partner. Even though there were differences in degrees, all of the participants showed the same patterns of transition of "Distinct impressions of partner" in the individual room environment. The first session had the lowest score and it showed an improvement in the second session. The improved score remained the same for the rest of the sessions. In the café environment, on the other hand, the amount of partner's self-disclosures and "distinct impressions of partner" were somewhat correlated. As mentioned above, the visibility of the avatar seemed to help participants to remember what they talked about and the information had accumulated throughout the sessions. In that case, without having visibility, accumulating the other party's information and forming district impressions would be very difficult. 
Similarity among non-native speakers. Both Jennifer and Mary rated higher on "sense of connection" in the café environment than the individual room environment while they rated higher on "comfort level of interaction" in the individual room environment in the first session. Jennifer explained that the avatar gave her "a better sense of connection" and felt "a stronger sense of personal interaction" during the first session in the café environment. Mary also mentioned that she felt "quite closely connected" to her partner during the first session in the café environment. On the other hand, when it comes to "comfort level of interaction," both of them preferred individual room. Jennifer said she "did not feel nervous about speaking with a stranger" and Mary also mentioned that she felt "slightly more comfortable" in the individual room after their first experience in both environment. Therefore, their comments show that they felt "sense of connection" in the café environment more while they felt higher "comfort level of interaction" in the individual room environment.

Difference among non-native speakers. Even though they showed similar preferences on "sense of connection" and on "comfort level of interaction," they showed differences on a preferred environment where they get to know someone for the first time and where they discus deeper issues. In Jennifer's opinion, interactions in the café environment were similar to those in real-life and she felt less pressure; hence, she preferred the café environment for meeting someone for the first time. Although Mary agreed with Jennifer about authenticity of interactions in the café environment, the same effect worked in the opposite direction. She mentioned that she was very invested in continuing the conversation and expressing herself and became less comfortable pausing or recovering from speech mistakes in the café. She also mentioned that if she meet someone for the first time, she would prefer to be slightly less self-conscious so that they can get to know each other better. 
For the environment where they discussed deeper issues, Jennifer explained that she thinks if one can see the other party they will be "on their best behavior and be civil and cordial about deeper issues" In addition to that, she felt safe if she can see the other party even virtually and the safe feeling would boost her confidence to discuss deeper issues."

On the contrary, Mary felt that the conversation in the café environment had been like an interview; therefore, she thought it would be harder to discuss deeper issues, as she felt greater pressure in continuing the conversation and did not want to say anything that would stop the conversation. One of the obvious factors would be their personalities. Some people prefer meeting another party in person to discuss something while others prefer contacting each other by e-mail or other forms of communication that does not require to be face-to-face. Another possible factor would be their speaking proficiencies. Mary expressed her fear of making mistakes in the café environment, which actually happened in the first session in the café environment. Jennifer made mistakes, too; however, her mistakes did not stop the conversation except for the "irumineeshon" incident, which was described in Excerpt 2.

Overall, Jennifer carried on the conversation fairly well throughout the sessions. Mary, on the other hand, had quite a number of fillers and constant self-corrections, which sometimes prevented a natural flow of the conversations. It is easy to guess that Mary had a higher selfconsciousness on making mistakes; therefore, the individual room environment was the better environment to cope with her self-consciousness. Considering that the native speakers, who typically do not have a fear of making language mistakes in their native language, prefer the café environment, Jennifer's preference could be similar to native speakers'.

Similarity among native speakers. Yoko and Keiko both expressed their uneasy feeling for not being able to see their partner's avatars throughout the sessions. In the individual room, 
Yoko often felt like she was talking to herself or simply throwing her words to the wall and that made her feel unsure of their conversation. She also sometimes lost her attention to her partner in the individual room because she could not find an "object" to talk to.

Likewise, Keiko had a feeling that "something was wrong" in the environment when she was not able to see her partner's face. She also mentioned that a sense of anxiety of whether her partner was there or not was depending on whether she could see her partner's avatar or not. It should be concluded, from what has been said above, that they both preferred to have visual representations of their partners to have conversations comfortably.

Difference among native speakers. The main difference was the ways of carrying the conversations. While Yoko tended to explain her answers extensively when she was asked questions, Keiko gave her partners short answers followed by her questions when she was asked questions. These tendencies were consistent from the first session through the last session regardless the environments; therefore, these are considered to be their ways of communication. This difference may explain how self-disclosures have progressed from the first to the last sessions. As for Yoko, the numbers of self-disclosures, especially level two self-disclosure show an increase throughout the sessions in both environments. This is because she added more feelings and values to her initial answers as the sessions progressed.

On the other hand, the number of Keiko's self-disclosures approximately remained the same throughout the sessions because she mainly asked questions than answering extensively. The number of AS-units actually show declines from the first to the last. Analyzing the transcript, this trend is likely due to the increase of the number of her partner's AS-units, including self-disclosures. Whether or not Keiko would keep the communication style or not if they kept the sessions is unknown. However, considering her experiences with communication 
breakdown, her comments about realizing how hard it is to express her thoughts, and not increasing the scores for emotional connections, it is very possible that she intentionally chose not to change her style and avoided giving self-disclosures.

Interestingly, her way of communication gave her partners positive impacts. Mary noticed that Keiko "said a little less" than Yoko; however, she still felt that both she and Keiko participated strongly in the conversation. The truth was, most of the time, Keiko gave Mary immediate feedback and asked her new questions but gave her much less self-disclosures compared to the amount Mary gave. As for Jennifer, she felt she connected better with Keiko and the amount of gaps in their conversation decreases every time. Therefore, this difference arose mainly from their personalities and personal preferences on the ways of carrying the conversations even though their experiences with their partners may enforce the tendencies

\section{Summary}

In this chapter, the results from the survey questions and conversation analysis were summarized under "1. Quantitative analysis for each participant to compare two environments" and "2. Qualitative analysis." These results were analyzed furthermore by each participant under "3. Integrative analysis for each participant" and "4. Further analysis for generalizability." From the results, five similarities among participants were derived: the effect of visibility of avatar, time to get to know someone, process of self-disclosure, environment where longer silences occur, and the transition of distinct impression of partner. The following chapter discusses the results from this chapter furthermore to answer research questions. 


\section{Chapter V: Conclusion}

\section{Overview}

This section discusses the findings of the data analysis performed in this study. The results from the previous section will be discussed furthermore to answer the research questions: how different visual design elements affect interpersonal emotional connections, what the impact that different visual design elements have on interpersonal emotional connections is, what the possible factors that affect interpersonal emotional connections are, and finally, what the relations between visual design elements and interpersonal emotional connections are.

\section{Discussion}

\section{RQ1 - How do different visual design elements of the environments of 3D VLEs}

\section{affect interpersonal emotional connections between participants in the initial stage}

of collaboration? First, participants' reflections and overlap of exchange sequence in the individual room environment indicate that the visual element of the avatar plays a role as a cue for remembering as discussed in Chapter III. Remembering and accumulating the other party's information is necessary to understand his/her views, perspectives and values through discussions; therefore, this function would be very beneficial in telecollaboration. This result matches the theory of social information processing, which was claimed by Walther (2002). According to Walther (2002), relationships grow only to the extent that participants first gain information about each other and they form interpersonal impressions of who they are with the information. Since participants in the individual room could not form their partner's interpersonal impressions without visual cues, they could not accumulate information that they gained in each session, and as a results, their relationship did not grow much. 
Second, without visual design elements of the other party, participants may feel less pressure to fill a silence. In other words, having visual design elements may give pressure to participants to avoid silences. How much effect that the environment may give on the length of silence remains to be proven because the results on Table 4 and 5 and examining participants' interactions indicate that conversation pattern is a stronger determiner of the silence. However, considering the outstanding amount of silence in the individual room, a comment from Mary mentioning that the individual room "felt less awkward," and a comment from Jennifer saying that people will be "on their best behavior" in the café environment, the environment may have impact on the amount and length of silence. How much effect that the environment may give on silence, however, remains to be proven.

Third, reflections from the participants indicate that the visual design elements work positively for native speakers in the initial stage of telecollaboration. They found similarities between having a conversation in an environment with the visual design elements and having a conversation in real life. The reflections also indicate that lack of visual design elements made them feel uneasy. In the individual room, Yoko felt like she was talking to herself and experienced losing attention to the conversation because there was no "object" to talk to. Keiko also felt "something was wrong" and felt anxious because she was not sure if her partner was there or not. Therefore, it is clear that they both would feel more comfortable if they had visual design elements of their partners.

Fourth, for non-native speakers, visual design elements may give them a sense of connection with the other party even though they may also have negative impacts on comfort level in the initial stage of telecollaboration. After the first sessions in the both environments, 
both of the non-native speakers rated higher on "sense of connection" while they rated lower on "comfort level of interaction" when they had the visual design elements.

Their comments prove the tendencies. Jennifer felt that the avatar gave her "a better sense of connection" and felt "a stronger sense of personal interaction." Mary also felt "quite closely connected" to her partner with the visual design elements. On the other hand, in the individual room, Jennifer did not feel nervous about speaking with a stranger" and Mary also felt "slightly more comfortable." Combining these comments and data from the graphs, it is evident that in the initial stage of collaboration, participants feel more "sense of connection" while they feel less "comfort level of interaction" when they had the visual design elements.

Finally, for non-native speakers who are worried about making mistakes, visual design elements work negatively on their comfort level so much that they prefer not to have them. As mentioned above, non-native speakers tend to feel more "sense of connection" and feel less "comfort level of interaction" with visual design elements in the initial stage of collaboration. However, if one is worried about making mistakes, he/she would value "comfort" than “connection" as Mary prefer the individual room over the café throughout the sessions.

RQ2-What is the impact that different visual design elements of the environments of

\section{D VLEs have on interpersonal emotional connections between participants in the initial}

stage of collaboration? First, combining the results from CA, social presence scores, and reflections from the participants, they seemed to receive an impact from from the environment strongly during the first session. However, by the third session, all of the participants became relatively comfortable with talking about recent events or their own cultures in both environments. After the third session, their emotional connections either became strong or 
became static. If there was no common interest or topics that they wanted to talk about, silences occurred more frequently in later sessions.

Second, even though the results from the social presence scores and reflections from the participants show that they received some impact from the environments, the number of selfdisclosures, which are considered to be the indication of an emotional connection between participants, did not receive major help from the environments. The number increased as the session progressed; that is, time is more crucial than the environment for the number of selfdisclosures.

Third, the results from social presence scores show that without visual design elements, one of the areas of emotional connection, "distinct impressions of partner" remained the same for the rest of the sessions after showing an improvement from the first to the second sessions. This is quite reasonable if an avatar has a role as a reminder of contents of the conversation. Without visual design elements, accumulating the other party's information and forming district impressions became difficult.

\section{RQ3-What are the possible factors that affect interpersonal emotional} connections between participants in the initial stage of collaboration in 3D VLEs? First, the reflections from the participants and looking at the relationship between topics of the conversations and survey results show that topics of the conversations have a great impact on emotional connections between participants. In the initial sessions, participants exchange basic information; therefore, they usually do not need to bring up topics to get to know each other. However, after several sessions, they need topics to talk about. If they choose wrong topics, such as topics that require difficult vocabulary items or topics that one of the participant does not have 
interest in, conversation may become dull and silences may arise and, as a result, negatively affect emotional connection between participants.

Second, conversation patterns also affect the impression of the conversation; therefore, they may affect emotional connections. It was observed that with adequate feedback and questions, the conversation keeps flowing naturally and that may affect the emotional connection between participants. As mentioned above, certain speech styles, such as inversion, could be confusing without nonverbal cues even though it is very common in natural conversation. These styles may cause misunderstandings, overlapping utterances and unwanted silences and eventually less emotional connection with their partners.

RQ4-What are the relations between visual design elements of the environments of 3D VLEs and interpersonal emotional connections between participants in the initial stage of collaboration? Combining the above answers for Research Questions 1, 2 and 3, the following relations become clear. For native speakers, visual design elements have positive impacts on emotional connections with their partners in the initial stage of the collaboration. However, as they continue on in their conversations, other factors, such as topics of the conversation, start to have a greater impact on emotional connections.

For non-native speakers, visual design elements may give them sense of connection with the other party even though the visual design elements may also have negative impact on their comfort level in the initial stage of collaboration. However, as is the case with native speakers, other factors start to have more impact on their emotional connections if they continue on their conversation.

For non-native speakers who have fears of making mistakes, visual design elements may cause greater uncomfortableness than sense of connection, which virtual design elements also 
give, and as a result, visual design elements may affect negatively on their emotional connections.

\section{Limitations}

There are four limitations, which were related to this study. First, since this study adapted single-subject research studies, it suffers from low external validity; therefore, results cannot be generalized to a population of interest. It is therefore necessary to conduct further studies to generalize an outcome of this study.

Second, even though the standards for determining levels of self-disclosures were established, judging if an AS-unit is a self-disclosure or not and judging its level are still fairly subjective. Two coders determined them separately; however, reconciled results could still be subjective. Therefore, the number of the self-disclosures in this study is rather relative than definite, such as the number of turns.

Third, in this study, all particpnats were female. Although a study showed that online social presence was not related to gender (Tu, Yen, \& Blocher, 2011), there might be a gender difference in perceptions of emotional connections. Therefore, conducting this study with all male participnats is necessary to generalize an outcome of this study.

Finally, during the sessions, there were technical problems and participants could not hear their partners from time to time. It is possible that these problems might affect participants' perceptions of emotional connections. Unless participants mentioned it in their reflections, separating factors, such as environments, topics, and technical problems, is impossible. Therefore, there is a possibility that the technical problems skewed the results. 


\section{Implications}

There are three implications, which should be helpful for using 3D VLEs. First, results showed that native speakers were more comfortable with communicating in 3D VLE with visual design elements than the one without. Therefore, using 3D VLEs may be a great opportunity for both native and non-native speakers to gather virtually and exchange opinions even though they are geographically dispersed in real-life.

However, it may not be true with learners with lower proficiency or leaners who are afraid of making mistakes. This is the second implication. The results show that for learners with lower proficiency or learners who are afraid of making mistakes, visual design elements of 3D VLEs may give negative impacts on their emotional connections with their partners or group members. One possible solution is giving those students less intimidating ways of communication, such as e-mails and text chat; this might be suitable to lower their anxieties.. However, does a "less intimidating environment" encourage learners as Salaberry (1996) pointed out? Warschauer (1997) also offers the example of Japanese students, who usually expected to be passive learners than being active in class, and claimed that CMC can offer these students opportunity to play an active role. However, as O'Dowd pointed out, the value of intercultural interaction which comes about through the disguising or hiding of aspects of one's identity is questionable (p. 80). All of the participants in this study admitted how meeting in the café environment is similar to meeting someone in the real world. If that is the case, it may be beneficial to overcome the fear, which learners may have when they meet someone from other countries, even in virtual environments.

Lastly, the results also showed that by the third sessions, participants became comfortable with talking about recent events or basic cultural information and they may not have topics to 
talk about if there is no common interest. In other words, they will be ready to move on to the next stage of the five-stage model proposed by Salmon (2000). After forming a solid social foundation, learners will be ready for more task-based information exchanges in stage 3, which is followed by knowledge construction in stage 4 (Salmon, 2011).

\section{Recommendations for further research}

First, as mentioned above, due to the low external validity, the results from this study cannot be generalized. Therefore replications of this study is necessary to generalize the results.

Second, the study dealt with pairs because of the technical restraints. Considering that telecollaboration is often designed as a group activity, knowing how group members socialize in the environment is crucial for successful activities. Therefore, further research is necessary to investigate how participants build emotional connections among members in a group setting.

Third, the results from this study indicate that proficiency may determine if visual design elements of 3D VLEs would be perceived beneficial or hindering. Therefore, further research also needs to look at learners with lower proficiency to clarify the effect of proficiency.

Fourth, participants in this study used avatars as visual cues to remember conversation with their partners; however, it is not clear if it has to be a 3D avatar. In the individual room, participants saw only their partner's names. Therefore, to clarify the effect of the avatars, study on participants in individual room with visual cues, such as icons, is necessary.

Fifth, it is quite possible that participants' personality may be a factor of preference of the environments in the initial stage. Therefore, further study on the relationship between personalities, such as extrovert vs. introvert, and their presence in the environment would be beneficial. 
Finally, as mentioned above, Keiko actively contributed to the conversations by giving short answers and asking further questions; however, the amount of self-disclosure and rating for emotional connections remained low throughout the sessions. The data and CA indicate that her conversation patters were fossilized by the fifth session and it is very likely that she would keep the pattern even if they continued the sessions. Since the main purpose of telecollaboration is to lead participants, both native and non-native, to develop intercultural communicative competence (ICC) (Byram, 1997) through interaction and exchange (Belz \& Thome, 2006), expressing their opinions and viewpoints is desirable. The previous studies mainly focused on the production of non-native speakers; however, research on native speakers' willingness to express their opinions is also valuable. Therefore, further study on the effective way to involve them into mutual conversations is desirable.

\section{Conclusions}

Advanced technology has given us abundant possibilities for foreign language education. It was not too long ago when one of the few choices that students could make to communicate with native speakers for deeper understanding their points of views and values was studying abroad. Thanks to advanced technology, the possibilities of meeting native speakers of a language, which one is studying, has broadened. As possibilities of using technology in foreign language education widen, more and more research has been done to show the possible affordance a certain technology could have, For example, Hedberg and Alexander (1994) identified the features of virtual environments and claimed that such environments have "the potential to offer a superior learning experience" (p. 218). Considering their social nature, 3D VLEs can potentially have useful social affordance. As Jarmon, Traphagan, Mayrath and Trivedi (2009) pointed out that the virtual platform of SL, especially various communication tools, 
provides opportunities for social interaction, collaboration, an increased sense of shared presence, partially dissolved social boundaries, and lowered social anxiety.

However, it is easy to imagine that not all of the learners will take advantage of these features equally. Some learners may find a certain affordance very useful while other learners may find it useless, or even worse, harmful. Therefore, it is crucial to know if learners will perceive affordance of technology and find it useful or not. Furthermore, knowing the process of utilizing the technology as well as hearing their voices are also important. There are many studies, which have been done to access the usefulness of various technology; however, many of them overlooked how each learner actually utilize it and how he/she perceive it during the process.

The results from this study show a variety of reflections and productions from the participants. No two individuals had the same reflections or production. However, by looking at their perceptions, production, and reflections, it became clear that they were all intertwined. During the process, the following points became clear:

First, learners may find avatars useful as a cue to remember the contents of the conversation.

Second, 3D VLEs may help native speakers or non-native speakers with higher proficiency to enforce emotional connections, which is important as Salmon (2000) said "success comes with a strong foundation at stage two (online socialization.)"

Third, for non-native speakers, 3D VLEs may bring positive effects, a sense of connection with their partners, and a negative effect, uncomfortableness. Whether the overall outcome turns out to be positive or negative may depend on a learner's personality and /or speaking proficiency. 
Finally, learners may find affordance of 3D VLEs useful in building emotional connections with their partners at the beginning stage of the collaboration; however, other factors, such as topic of the conversation, gain impacts on emotional connections as the collaboration goes on.

Therefore, educators should first consider various factors of their students, such as their proficiency, length of the project, and design of the project, very carefully if they plan to use 3D VLEs for telecollaboration.

Technology will surely advance in the future. We, educators, however, always keep in mind the things that technology can offer is not necessarily useful for learners even if it is useful in other places. We should not overlook the learner's perceptions, production, and reflections when using technology for education. 


\section{References}

Abeele, M. V., Roe, K., \& Pandelaere, M. (2007, October). Construct validation of the concepts social presence, emotional presence, and connectedness and an application of Zajonc's social facilitation theory to social presence research. Proceedings of the 10th annual international workshop on Presence, Barcelona, Spain.

Altman, I., \& Taylor, D., (1973). Social Penetration: The Development of Interpersonal Relationships. NewYork: Holt, Rinehart and Winston

Antaki, C., Barnes, R., \& Leudar, I. (2005). Self-disclosure as a situated interactional practice. British Journal of Social Psychology, 44, 181-199

Anthes, G. (2007, December 3). Second Life: Is there any there there? Computerworlds, 41(49), $30-37$.

Antonacci, D., \& Modaress, N. (2005). Second Life: The educational possibilities of a massively multiplayer virtual world (MMVW). Retrieved December 21, 2011, from http://net.educause.edu/ir/library/pdf/WRC0541.pdf

Armstrong, K. and O. Retterer (2008) Blogging as L2 writing: a case study. AACE Journal 16 (3), 233-251

Au, W. J. (2008) The Linden Billboards regulations seem to be working, but are they enough to make the SL land appealing? Retrieved December 21, 2011, from http://nwn.blogs.com/nwn/2008/10/as-advertised-f.html

Belz, J. A. (2005). Intercultural questioning, discovery and tension in Internet-mediated language learning partnerships. Language and Intercultural Communication, 5 (1), 3-39. 
Belz, J. A. \& Thorne, S. L. (2006). Introduction: Internet-Mediated Intercultural Foreign Language Education and the Intercultural Speaker. In J. A. Belz \& S. L. Thorne (Eds.), Internet-Mediated Intercultural Foreign Language. Boston, MA: Heinle \& Heinle.

Bowers, K.W. Ragas, M.W., \& Neely, J.C. (2009). Assessing the value of virtual worlds for post-secondary educators: A survey of innovators, early adopters and the early majority in Second Life. International Journal of Humanities and Social Sciences, 3(1), 40-50.

Brna, P. (1999). Collaborative virtual learning environments for concept learning. International Journal of Continuing Education and Lifelong Learning, 9, 3/4, 315-327.

Byram, M. (1997), Teaching and Assessing Intercultural Communicative Competence. Clevedon: Multilingual Matters.

Bente, G.,Rüggenberg, S., Kräamer, N.(2004). Social presence and interpersonal trust in avatarbased, collaborative net-communications," in 7th Annual International Workshop on Presence, 14 .

Bonk, C. J., Hansen, E. J., Grabner-Hagen, M. M., Lazar, S. A., \& Mirabelli, C. (1998). Time to “connect": Synchronous and asynchronous dialogue among preservice teachers. In C. J. Bonk \& K. S. King (Eds.), Electronic collaborators: Learner-centered technologies for literacy, apprenticeship, and discourse, (pp. 289-314). Mahwah, NJ: Erlbaum.

Bowers, K.W. (2010). Constructing the new classroom: College student perceptions of classes using Second Life. Retrieved from ProQuest Dissertations and Theses. (AAT 3467662)

Bowers,W., Davis, D., \& Neely, J. (2009). Framing Second Life for use in higher education: An analysis of EDUCAUSE Review and The Chronicle of Higher Education. The Journal of Virtual Worlds and Education, 1(1), 136-167. 
Branon, R. F., \& Essex, C. (2001). Synchronous and asynchronous communication tools in distance education: A survey of instructors. TechTrends, 45, 36-42.

Bugeja, M. J. (2007). Second thoughts about Second Life. The Chronicle of Higher Education, 54(3), C1.

Burnett, C. (2003). Learning to chat: Tutor participation in synchronous online chat. Teaching in Higher Education, 8, 247-261.

Carlorosi,S., Helm, F., Marini-Maio , N., \& McMahon, K. (2008). Confronting New Technologies: a Cross-Cultural Telecollaborative. Project across the Ocean. In E. Occhipinti (Ed.), New Approaches to Teaching Italian Language and Culture: Case Studies from an International Perspective, (pp. 173-213). Newcastle upon Tyne, UK: Cambridge Scholars Publishing

Cheal, C. (2007). Second Life: Hype or hyperlearning? On the Horizon, 15(4), 204-210.

Chen, Y.W., \& Nakazawa, M. (2012). Measuring patterns of self-disclosure in intercultural friendship: Adjusting differential item functioning using multiple-indicators, mulitplecauses models. Journal of Intercultural Communication Research, 41(2), 131-151.

Childress, M., \& Braswell, R. (2006). Using massively multiplayer online role-playing games for online learning. Distance Education, 27(2), 187.

Chittaro, L., Ranon, R., 2007. Web3D technologies in learning, education and training: motivations, issues, opportunities. Computers \& Education, 49(1), 3-18.

Christophel, D. M. (1990). The relationships among teacher immediacy behaviors, student motivation, and learning. Communication Education, 39, 323-340.

Commission of the European Communities (2003), Communication from the commission to the council and the Europian Parliament. Development of the Schengen Information System 
II and possible synergies with a future Visa Information System (VIS)', COM, 771 final. Retrived from http://eur-lex.europa.eu/LexUriServ/LexUriServ.do?uri=COM: 2011:0771: FIN:EN:PDF

Conklin, M. S. (2007). 101 uses for Second Life in the college classroom. Retrieved from http://citeseerx.ist.psu.edu/viewdoc/download?doi=10.1.1.133.9588\&rep $=$ rep $1 \&$ type $=$ pdf

Cook, S. D. \& Brown, J. S. (1999). Bridging Epistemologies: The Generative Dance between Organizational Knowledge and Organizational Knowing. Organization Science, 10, 381400.

Creswell, J. W. (2009). Research design: Qualitative, quantitative, and mixed methods approaches (3rd ed.). Los Angles: Sage.

Creswell, J.W., \& Plano Clark, V.L. (2007). Designing and conducting mixed methods research. Thousand Oaks, CA: Sage.

Daft, R. L., \& Lengel, R. H. (1984). Information richness: A new approach to managerial behavior and organizational design. In L. L. Cummings \& B. M. Staw (Eds.), Research in organizational behavior, 191-233. Homewood, IL: JAI Press.

Daft, R. L., \& Lengel, R. H. (1986). Organizational information requirements, media richness and structural design. Management Science, 32(5), 554-571.

Dalgarno, B. \& Lee, M.J.W. (2010). What are the learning affordances of 3D virtual environments? British Journal of Educational Technology, 40(6), 10-32.

Davidson-Shivers, G. V., Muilenburg, L. Y., \& Tanner, E. J. (2001). How do students participate in synchronous and asynchronous online discussions? Journal of Educational Computing Research, 25, 351-366. 
Dawson, S. (2006). Relationship between student communication interaction and sense of community in higher education. Internet and Higher Education, 9(3), 153-162.

de Greef, P. \& IJsselsteijn, W.A. (2001). Social presence in a home teleapplication. CyberPsychology and Behavior 4, 307-315.

De Lucia, A., Francese, R., Passero, I., Tortora, G.(2009). Development and evaluation of a virtual campus on second life: The case of second DMI. Computer and Education, 52(1), $220-233$.

Dennis, A. R., Fuller, R. M., \& Valacich, J. S. (2008). Media, tasks, and communication processes: a theory of media synchronicity. MIS Quarterly, 32(3), 575-600.

Derlega, V. J., Metts, S., Petronio, S., \& Margulis, S. T. (1993). Self-disclosure. Newbury Park, CA: Sage.

Dindia, K. (2000). Relational maintenance. In C. Hendrick \& S. Hendrick (Eds). Close relationships: A sourcebook, (pp. 287-300). Thousand Oaks, CA: Sage.

Dickey, M. D. (2005). Three-dimensional virtual worlds and distance learning: two case studies of Active Worlds as a medium for distance education. British Journal of Educational Technology, 36(3), 439-451.

Dickey, M.,D. (2003). Teaching in 3D: Pedagogical affordances and constraints of 3D virtual worlds for synchronous distance learning. Distance Education, 24(1), 105-121.

Dieterle, E., \& Clarke, J. (2009). Multi-user virtual environments for teaching and learning. In M. Pagani (Ed.), Encyclopedia of Multimedia Technology and Networking (2nd ed.), Vol II (pp. 1033-1041). Hershey, PA: Information Science Reference.

Duffy, Thomas M. \& Kirkley, Jamie R. (2004) Learning Theory and Pedagogy Applied in Distance Learning: The Case of Cardean University. In T. Duffy \& J. Kirkley (Eds) 
Learner Centered Theory and Practice in Distance Education: Cases from Higher Education. Mahwah, NJ: Lawrence Erlbaum and Associates.

Foster, A. L. (2007, September 21). Professor avatar. Chronicle of Higher Education, 54(4). Retrieved from http://chronicle.com/article/Professor-Avatar/30018/

Foster, P., Tonkyn, A. \& Wigglesworth, G. (2000) Measuring spoken language: A unit for all reasons. Applied Linguistics 21(3), 354-375.

Fulk, J., Schmitz, J. \& Steinfeld, C. W. (1990). A Social Modell of Technology Use. In J. Fulk \& C. W. Steinfeld (Eds.), Organizations and Communication Technology (pp. 117-140). Newbury Park: Sage.

Gay, L. R. \& Airasian, P. (2003). Educational research: Competencies for analysis and applications (7th ed.). Upper Saddle River, NJ: Pearson Education.

Garrison, D. R. (2007). Online community of inquiry review: Social, cognitive, and teaching presence issues. Journal of Asynchronous Learning Networks, 11(1), 61-72.

Garrison, D. R. \& Cleveland-Innes, M. (2005). Facilitating Cognitive Presence in Online Learning: Interaction Is Not Enough. American Journal of Distance Education, 19(3), 133-148.

Garrison, D. R., Anderson, T., \& Archer, W. (2000). Critical inquiry in a text-based environment: Computer conferencing in higher education. The Internet and Higher Education, 2(2-3), 87-105.

Garrison, D. R., Anderson, T., \& Archer, W. (2001). Critical thinking, cognitive presence, and computer conferencing in distance education. American Journal of Distance Education, $15(1), 7-23$. 
Gast, D. L. (2010). Single Subject Research Methodology in Behavioral Sciences. New York, NY: Routledge.

Gibson, J. (1979). The Ecological Approach to visual perception. Boston: Houghton Mifflin

Gorham, J. (1988). The relationship between verbal teacher immediacy behaviors and student learning. Communication Education, 37, 40-53.

Gordon, I.E. (1989). Theories of visual perception. Chichester, UK: John Wiley and Sons.

Gray, B. (2004). Informal Learning in an Online Community of Practice. Journal of Distance Education/Revue de l'enseignement à distance, 19(1), 20-35.

Gunawardena, C. N. (1995). Social presence theory and implications for interaction and collaborative learning in computer conferences. International Journal of Educational Telecommunications, 1(2/3), 147-166.

Gunawardena, C. N., \& Zittle, F. J. (1997). Social presence as a predictor of satisfaction within a computer-mediated conferencing environment. American Journal of Distance Education, $11(3), 8-26$.

Haneda, M. (1997). Second language learning in a "community of practice": A case study of adult Japanese learners. The Canadian Modern Language Review, 54, 11-27.

Harms, C., \& Biocca, F. (2004). Internal consistency and reliability of the networked minds measure of social presence. Proceedings of the Seventh Annual International Presence Workshop. Valencia, Spain: Universidad, 246-251

Haythornthwaite, C. \& Kazmer, M. M. (2002). Bringing the Internet home: Adult distance learners and their Internet, Home and Work worlds. In B. Wellman \& C. Haythornthwaite (Eds.), The Internet in Everyday Life (pp. 431-463). Oxford, UK: Blackwell. 
Hedberg, J.G., \& Alexander, S. (1994). Virtual Reality in Education: Defining researchable issues.Educational Media International, 31(4), 214-220.

Henri, F. (1992). Computer conferencing and content analysis. In A. R. Kaye (Ed.), Collaborative learning through computer conferencing (pp. 117-136). The Najaden Paper. New York: Springer.

Hornik, S. (2008). Constructivist learning in Second Life: Empirical evidence beating the challenges, and best practices in design and development. Retrieved from http://www.aect.org/SecondLife/08-archives.asp

Hostetter, C., \& Busch, M. (2006). Measuring up online: The relationship between social presence and student learning satisfaction. Journal of Scholarship of Teaching and Learning, 6(2), 1-12.

Hughes, M., Ventura, S., \& Dando, M. (2007). Assessing social presence in online discussion groups: A replication study. Innovations in Education and Teaching International, 44(1), $17-29$.

Jamaludin, A., Chee, Y., \& Ho, C. (2009). Fostering argumentative knowledge construction through enactive role play in Second Life. Computers \& Education, 53(2), 317-329

Jarmon, L., Traphagan, T., Mayrath, M., \& Trivedi, A. (2009). Virtual world teaching, experiential learning, and assessment: An interdisciplinary communication course in Second Life. Computers \& Education, 53(1), 169-182.

Jourard, S.M., \& Lasakow, P. (1958). Some factors in self-disclosure. Journal of Abnormal and Social Psychology, 56, 91-98.

Kalyuga, S. (2007). Enhancing instructional efficiency of interactive e-learning environments: A cognitive load perspective. Educational Psychology Review, 19(3), 387-399. 
Kasper, G. (1997). “A” stands for acquisition: A response to Firth and Wagner. Modern Language Journal, 81, 307-312.

Kennedy, C. (2005). Single-case designs for educational research. Boston: Allyn \& Bacon.

Kiesler, S., Siegel, J., \& McGuire, T. W. (1984). Social psychological aspects of computermediated communication. American Psychologist, 39(10), 1123-1134.

Kikukawa, H. (2012).Taiwanji JFL kyoshitsu shutokusha no nihongo hattatsu [The developmental process of Taiwanese JFL learners]. (Doctoral dissertation). Retrived from http://163.14.136.66:8080/retrieve/558/100SCU05079001-001.pdf

Kinginger, C.(2004). Alice doesn't live here anymore: Foreign language learning and identity reconstruction. In A. Pavlenko \& A. Blackledge (Eds.), Negotiation of identities in multilingual contexts (pp. 219-42). Clevedon: Multilingual Matters.

Kirkpatrick, D. (2007, January 23). Second Life: It's not a game. Fortune. Retrieved from http://money.cnn.com/2007/01/22/magazines/fortune/whatsnext_secondlife.fortune/index .htm

Kirschner, P., Strijbos, J., Kreijns, K., \& Beers, P. J. (2004). Designing electronic collaborative learning environments. Educational Technology Research and Development, 52(3), 4766.

Kramer, A. D. I., Oh, L. M., \& Fussell, S. R. (2006). Using linguistic features to measure presence in computer-mediated communication. In Proceedings of the SIGCHI conference on Human Factors in Computing Systems (pp. 913-916). New York: ACM Press. 
Kreijns, K., Kirschnerb, P.A.\& Jochems, W. (2003, May). Identifying the pitfalls for social interaction in computer-supported collaborative learning environments: a review of the research, Computers in Human Behavior, 19, 3, 335-353.

Kreijns, K., Kirschner, P., \& Jochems, W. (2002). The sociability of computer-supported collaborative learning environments. Educational Technology \& Society, 5(1), 8-25.

Krippendorf, K. (1989). On the essential contexts of artifacts or on the proposition that 'design is making sense (of things). Design Issues, 5(2), 9-39.

Kuhn, T.,S. (1996). The structure of scientific revolutions (3rd ed.). Chicago, IL: University of Chicago Press.

Laurillard, D., Stratfold, M., Luckin, R., Plowman, L. \& Taylor, J. (2000). Affordances for Learning in a Non-Linear Narrative Medium. Journal of Interactive Media in Education, 2000(2). Retrieved from http://www-jime.open.ac.uk/00/2

Lin, G. Y. (2004, October). Social presence questionnaire of online collaborative learning: Development and validity. Paper presented at the annual meeting of the Association for Educational Communications and Technology, Chicago, IL.

Lowenthal, P. R. (2009). The evolution and influence of social presence theory on online learning. In T. T. Kidd (Ed.), Online education and adult learning: New frontiers for teaching practices. Hershey, PA: IGI Global.

Ludlow, P. \&Wallace, M. (2007). The Second Life Herald-the virtual tabloid that witnessed the dawn of the metaverse. Cambridge, MA: MIT press.

Ludwig-Hardman S., \& Dunlap, J. C. (2003). Learner support services for online students: scaffolding for success. The International Review of Research in Open and Distant Learning, 4(1). 
Maezawa, A. (2008). The adjustments to make close relationship between Japanese students and international students. Chiba University Repository for Access to Outcomes from Research, 6, 29 - 45

McKerlich, R., \& Anderson, T. (2007). Community of inquiry and learning in immersive environments. Journal of Asynchronous Learning Networks, 11(4), 35-52.

McLellan, H. (2004). Virtual realities. In D. H. Jonassen (Ed.), Handbook of research for educational communications and technology (2nd ed., pp. 461-497), Mahwah, NJ: Lawrence Erlbaum.

Meadows, M., S. (2008). I, avatar: The culture and consequences of having a Second Life. Berkeley, CA: New Riders Press

Mikropoulos, T. A. (2006). Presence: a unique characteristic in educational virtual environments. Virtual Reality, 10, 3/4, 197-206.

Mikropoulos, T. A. \& Strouboulis, V. (2004). Factors that influence presence in educational virtual environments. CyberPsychology \& Behavior, 7, 5, 582-591.

MLA Ad Hoc Committee on Foreign Languages. (2007). Foreign languages and higher education: New structures for a changed world. Retrieved from http://www.mla.org/pdf/forlang_news_pdf.pdf

Molinari, D., L. (2004). The role of social comments in problem-solving groups in an online class. American Journal of Distance Education, 18(2), 89-101.

Monahan, T., McArdle, G., Bertolotto,M. (2008). Virtual Reality for Collaborative e-Learning, Journal of Computers and Education, 50, 1339-1353.

Murphy, E (2004) Recognising and promoting collaboration in an online asynchronous discussion. British Journal of Educational Technology, 35(4), 421-431 
Murphy, E., \& Laferriére, T. (2007). Adopting tools for online synchronous communications: issues and strategies. In M. Bullen, \& D. Janes (Eds.), Making the transition to Elearning: Strategies and issues (pp. 313-334). Hershey, PA: Idea Group.

Nakayama, A. (2008). The communication of closeness. Kuroshio. Tokyo, Japan

Neely, J.C., Bowers, K.W. \& Ragas, M.W. (2010). Virtual Possibilities: A Constructivist Examination of the Educational Applications of Second Life. Journal of Interactive Learning Research, 21(1), 93-110. Chesapeake, VA: AACE.

Norman, D. (2004). Emotional design: Why we love (or hate) everyday things. New York: Basic Books.

Norman, D. (1998). The psychology of everyday things. New York: Basic Books.

Northrup, P. T. (2002). Online learners' preference for interaction. The Quarterly Review of Distance Education, 3(2), 219-226.

Nowak, K. (2001). Defining and Differentiating Copresence, Social Presence and Presence as Transportation. Paper presented at the Presence 2001Conference, Philadelphia, PA.

Nowak, K.L., \& Biocca, F. (2003). The effect of the agency and anthropomorphism on users' sense of telepresence, copresence, and social presence in virtual environments. Presence: Teleoperators and Virtual Environments, 12, 2-35

O'Dowd, R (2006) Telecollaboration and the development of intercultural communicative competence. Berlin: Langenscheidt

O'Dowd, R. and Ritter, M. (2006) Understanding and working with 'failed communication' in telecollaborative exchanges. CALICO Journal, 61(2), 623-642. 
Picciano, A. (2002). Beyond student perceptions: Issues of interaction, presence, and performance in an online course. Journal of Asynchronous Learning Networks, 6(1), 2140.

Poole, D. M. (2000). Student participation in a discussion-oriented online course: A case study. Journal of Research on Computing in Education, 33(2), 162-177.

Poulisse, N. (1997). Some words in defense of the psycholinguistic approach: A response to Firth and Wagner. Modern Language Journal, 81, 324-328.

Preece, J. (2000). Online Communities: Designing Usability, Supporting Sociability. Chichester, UK: John Wiley \& Sons

Reiser, R., \& Dempsey, J. (2012). Trends and issues in instructional design and technology. (3rd ed.). Boston, MA: Pearson/Prentice Hall

Richardson, J. C., \& Swan, K. (2003). Examining social presence in online courses in relation to students' perceived learning and satisfaction. Journal of Asynchronous Learning Networks, 7(1), 68-88.

Richmond, V. P. (1990). Communication in the classroom: Power and motivation. Communication Education, 39,181-195.

Robert, L.P., \& Dennis, A.R. (2005). Paradox of richness: A cognitive model of media choice. IEEE Transactions on Professional Communication, 48(1), 10-21.

Rodriguez, J., Plax, T. G., \& Kearney, P. (1996). Clarifying the relationship between teacher nonverbal immediacy and student cognitive learning: Affective learning as the central casual mediator. Communication Education, 45, 293-305. 
Romiszowski, A., \& Mason, R. (2004). Computer-mediated communication. In D. H. Jonassen (Ed.), Handbook of research for educational communications and technology. New Jersey: Lawrence Erlbaum.

Rourke, L., Anderson, T., Garrison, D. R., \& Archer, W. (1999). Assessing social presence in asynchronous text-based computer conferencing. Journal of Distance Education, 14(3), 51-70.

Sacks, H. (1992). April 24: Second stories. In G. Jefferson (Ed.), Lectures on conversation (pp. 764-772). Cambridge, MA: Blackwell Publishers.

Salmon, G. (2000). E-moderating: The key to teaching and learning online. New York: Routledge.

Salmon. G., Nie, M., \& Edirisingha, P. (June, 2010). Developing a five-stage model of learning in Second Life. Educational Research, 52(2), 169-182.

Schroeder, R. (2002). Social interaction in virtual environments: key issues, common themes, and a framework for research. In R. Schroeder (Ed.), The social life of avatars: presence and interaction in shared virtual environments (pp. 1-18). London: Springer.

Schroeder, R. \& Axelsson, A. (Eds) (2006). Avatars at work and play: collaboration and interaction in shared virtual environments. London: Springer-Verlag.

Schroeder, R. (1996). Possible Worlds: The Social Dynamic of Virtual Reality Technologies. Boulder: Westview Press.

Short, J., Williams, E., \& Christie, B. (1976). The social psychology of telecommunications. London: John Wiley \& Sons.

Sidnell, J. (2010) Conversation analysis: An introduction. Chichester, U.K.: Wiley-Blackwell. 
Slater, M. (1999). Measuring presence: a response to the Witmer and Singer questionnaire. Presence, 8, 5, 560-566.

Stein, D. S., \& Wanstreet, C. E. (2003). Role of social presence, choice of online or face-to-face group format, and sat with perceived knowledge gained in a distance learning environment. Paper presented at the Midwest Research to Practice Conference in Adult, Continuing, and Community Education, Columbus, $\mathrm{OH}$.

Steinfield, C.W. (1986). Computer mediated communication in an organizational setting: Explaining task-related and socioeconomical uses. In M.L. Mclaughlin (Ed.) Communication yearbook, 9 (pp. 777-804). Newbury Park, CA: Sage.

Swan, K. (2002). Building learning communities in online courses: the importance of interaction, Education, Communication \& Information, 2(1), pp. 23-49.

Swan, K. (2003). Developing social presence in online course discussions. In S. Naidu (Ed.), Learning and teaching with technology: Principles and practices (pp. 147-164). London: Kogan Page.

Swan, K., \& Shea, P. (2005). The development of virtual learning communities. In S. R. Hiltz \& R. Goldman (Eds.), Asynchronous learning networks (pp. 239-260). New York: Hampton Press.

Swan, K., Richardson, J., Ice, P., Shea, P., Cleveland-Innes, M., Diaz, S. \& Garrison, R. (2008). Researching Online Communities of Inquiry: New CoI Survey Instrument. In J. Luca \& E. Weippl (Eds.), Proceedings of World Conference on Educational Multimedia, Hypermedia and Telecommunications 2008 (pp. 5812-5820). Chesapeake, VA: AACE.

Tu, C. H. (2002). The measurement of social presence in an online learning environment. International Journal on E-Learning, 1(2), 34-45. 
Tu, C. H., \& McIsaac, M. (2002). The relationship of social presence and interaction in online classes. American Journal of Distance Education, 16(3), 131-150.

Tu, C. H., Yen, C., \& Blocher, M. (2011). A Study of the Relationship between Gender and Online Social Presence. International Journal of Online Pedagogy and Course Design (IJOPCD), 1(3), 33-49.

Tuovinen, J. E. (2000). Factors influencing the success of computer mediated communication (CMC) environments in university teaching: A review and case study. Educational Media International, 37(2), 16-24.

Valenta, A., Therriault, D., Dieter, M., \& Mrtek, R. (2001). Identifying student attitudes and learning styles in distance education. Journal of Asynchronous Learning Networks, 5(2), 111-127.

Walther, J. B. (1992). Interpersonal effects in computer-mediated interaction: A relational perspective. Communication Research, 19, 52-90.

Walther, J. B. (1996). Computer-mediated communication: Impersonal, interpersonal, and hyperpersonal interaction. Communication Research, 23(1), 3-43.

Walther, J. B., \& Parks, M. R. (2002). Cues filtered out, cues filtered in: Computer-mediated communication and relationships. In M. L. Knapp \& J. A. Daly (Eds.), Handbook of interpersonal communication ( $3^{\text {rd }}$ ed., pp. 529-563). Thousand Oaks, CA: Sage.

Wang, Y., \& Chen, N. S. (2007). Online synchronous language learning: SLMS over the Internet. Innovate: Journal of Online Education, 3(3), Retrieved October 18, 2011 from.http://www.innovateonline.info/pdf/vol3_issue3/Online_Synchronous_Language_L earning-_SLMS_over_the_Internet.pdf 
Wang, Y., Chen, N. S., \& Levy, M. (2010). Teacher training in a synchronous cyber face-to-face classroom: characterizing and supporting the online teachers' learning process. Computer Assisted Language Learning, 23(4), 277-293.

Warburton, S. (2009) Second Life in higher education: Assessing the potential for and the barriers to deploying virtual worlds in learning and teaching.British Journal of Educational Technology, 40(3), 414-426.

Warburton, S. \& Pérez Garcia, M. (2009). 3D design and collaboration in massively multi-user virtual environments. In D. Russell (Ed.), Cases on collaboration in virtual learning environments:processes and interactions. Hershey, PA: IGI Global.

Warschauer, M. (2000). The death of cyberspace and the rebirth of CALL. English Teachers Journal, 53, 61-67.

Weiner, B., Johnson, P., \& Mehrabian, A. (1968). Achievement motivation and the recall of incompleted and completed exam questions. Journal of Educational Psychology, 59, $181-185$.

Wenger, E. (2001). Supporting communities of practice: A survey of community-oriented technologies. Retrieved from http://www.ewenger.com/ tech/index.htm

Wenger, E., McDermott, R. A., \& Snyder, W. (2002). Cultivating communities of practice : a guide to managing knowledge. Boston: Harvard Business School Press.

Wiener, M.,\&Mehrabian, A. (1968). Language within language:Immediacy, a channel in verbal communication. New York: Appleton-Century-Crofts.

Wise, A., Chang, J., Duffy, T., \& Del Valle, R. (2004). The effects of teacher social presence on student satisfaction, engagement, and learning. Journal of Educational Computing Research, 31(3), 247-271. 
Witmer, D. F. (1997). Risky business: Why people feel safe in sexually explicit on-line communication. Journal of Computer Mediated Communication, 2(4), 13-24.

Witmer, B. G. \& Singer, M. J. (1998). Measuring presence in virtual environments: a presence questionnaire. Presence, 7, 3, 225-240.

Whitelock, D., Brna, P. \& Holland, S. (1996). What is the value of virtual reality for conceptual learning? Towards a theoretical framework. In P. Brna, A. Paiva \& J. A. Self (Eds), Proceedings of the European Conference on artificial intelligence in education (EuroAIE) (pp. 136-141). Lisbon: Edicões Colibri

Wuensch, K. L., Aziz, S., Ozan, E., Kishore, M., \& Tabrizi, M. H. N. (2008). Pedagogical characteristics of online and face-to-face classes. International Journal on E-Learning, 7, 523-532.

Yamada,M., Akahori,K. (2007) An Analysis of the relationship between presence, consciousness and performance in learner-centered communicative learning using SCMC- Experimental study. Journal of Asia TEFL,4(4), 59-91.

Yamada, M., \& Kitamura, S. (2010) A Review of "Social Presence” for CSCL Research. Japan Journal of Educational Technology, 33(3), 353-362. 


\section{Appendix A}

Survey for each session

(Questionnaire items for social presence (adapted from Swan et al (2008))

1. University

$\square \quad$ Princeton University

$\square \quad$ Waseda University

2. Which group were you in?

$\square \quad$ Group A

$\square \quad$ Group B

$\square \quad$ Group C

$\square \quad$ Group D

3. This was my time (s) in this group.

$\square \quad$ First

$\square \quad$ Second

$\square \quad$ Third

$\square \quad$ Fourth

$\square \quad$ Fifth 
Please indicate the degree to which you Agree/Disagree with each statement as it relates to your experience in each session.

4. Getting to know my partner in this environment gave me a sense of connection with my partner.

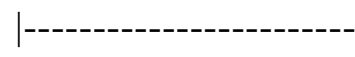

Strongly disagree Disagree

Neutral

Agree

Strongly agree

5. I was able to form distinct impressions of my partner through the online medium in this environment.

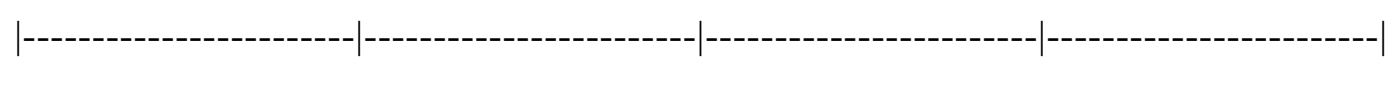

Strongly disagree Disagree Neutral Agree Strongly agree

6. This form of online communication in this environment is an excellent medium for social interaction.

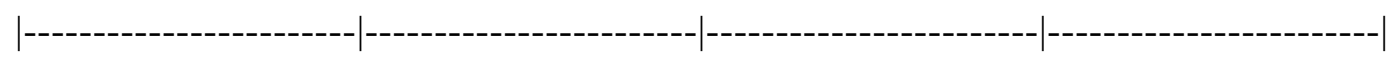

Strongly disagree Disagree Neutral Agree $\quad$ Strongly agree

7. I felt comfortable interacting with my partner in this environment.

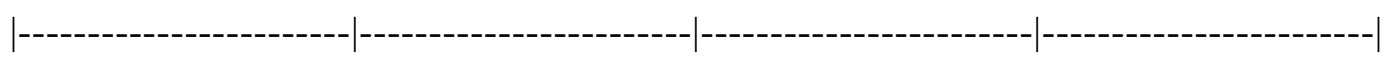

Strongly disagree Disagree Neutral Agree $\quad$ Strongly agree

8. I felt comfortable disagreeing with my partner in this environment while still maintaining sense of trust.

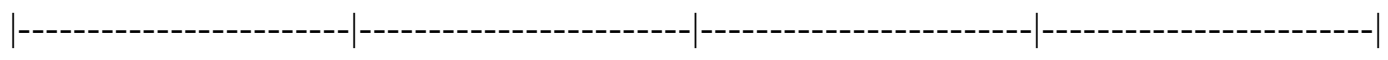

Strongly disagree Disagree Neutral Agree $\quad$ Strongly agree

9. I felt that my point of view was acknowledged by my partner in this environment.

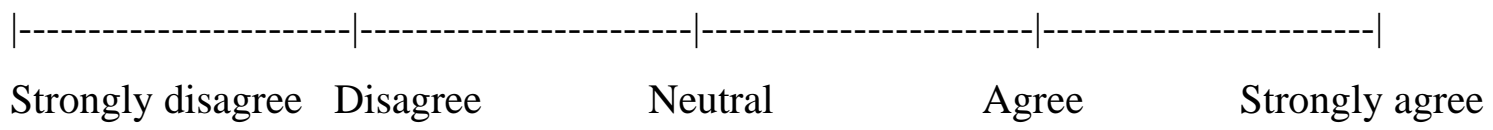


10. Online discussions in this environment help me to develop a sense of collaboration.

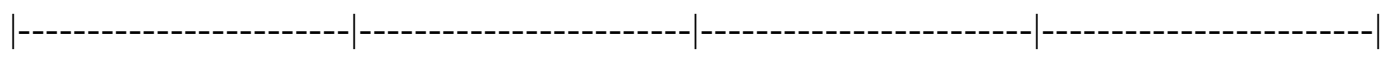

Strongly disagree Disagree $\quad$ Neutral Agree $\quad$ Strongly agree

(For the first session)

Please write a brief impression of the session:

(For the second to fifth sessions)

Please write a brief impression of the session. If you think you have more or less of an emotional connection with your partner than last time, what do you think makes you feel that way? 


\section{Appendix B}

Survey after the first set of sessions

1. University

$\square \quad$ Princeton University

$\square \quad$ Waseda University

2. Which groups were you in?

$\square$ Group A

$\square \quad$ Group B

$\square$ Group C

$\square$ Group D

3. Was this the first time to use Second Life? ( Yes / No )

4. Did you find any difference between conversation in the café and that in the individual room?

( Yes / No ) If the answer is "Yes", please answer question \#5.

5. What were the differences?

6. Which environment did you prefer to get to know someone you've never met in real life?

( Café / Individual room / Environment doesn't matter )

>Please tell me why you think so. 


\section{Appendix C}

Survey after all of the sessions

\section{University}

$\square \quad$ Princeton University

口 Waseda University

2. Which groups were you in?

$\square$ Group A

$\square$ Group B

$\square \quad$ Group C

$\square \quad$ Group D

3. Did you find any difference between conversation in the café and that in the individual room?

( Yes / No ) If the answer is "Yes," please answer question \#4. $>$ Please tell me why you think so.

4. What were the differences?

5. Which environment did you prefer to get to know someone you've never met in real life?

( Café / Individual room / Environment doesn't matter ) >Please tell me why you think so.

6. Which environment do you think you would feel more comfortable if you would have to discuss deeper issues, such as your values, attitudes, conceptions, and beliefs underlying your culture, with someone you've never met in real life?

( Café / Individual room / Environment doesn't matter ) >Please tell me why you think so.

Thank you for your cooperation! 


\section{Appendix D}

\section{ACTFL (American Council on the Teaching of Foreign Languages) Proficiency Guide 2012-Speaking}

\section{INTERMEDIATE}

Speakers at the Intermediate level are distinguished primarily by their ability to create with the language when talking about familiar topics related to their daily life. They are able to recombine learned material in order to express personal meaning. Intermediate- level speakers can ask simple questions and can handle a straightforward survival situation. They produce sentence-level language, ranging from discrete sentences to strings of sentences, typically in present time. Intermediate-level speakers are understood by interlocutors who are accustomed to dealing with non-native learners of the language.

\section{Intermediate High}

Intermediate High speakers are able to converse with ease and confidence when dealing with the routine tasks and social situations of the Intermediate level. They are able to handle successfully uncomplicated tasks and social situations requiring an exchange of basic information related to their work, school, recreation, particular interests, and areas of competence.

Intermediate High speakers can handle a substantial number of tasks associated with the Advanced level, but they are unable to sustain performance of all of these tasks all of the time. Intermediate High speakers can narrate and describe in all major time frames using connected discourse of paragraph length, but not all the time. Typically, when Intermediate High speakers attempt to perform Advanced-level tasks, their speech exhibits one or more features of breakdown, such as the failure to carry out fully the narration or description in the appropriate major time frame, an inability to maintain paragraph-length discourse, or a reduction in breadth and appropriateness of vocabulary.

Intermediate High speakers can generally be understood by native speakers unaccustomed to dealing with non-natives, although interference from another language may be evident (e.g., use of code-switching, false cognates, literal translations), and a pattern of gaps in communication may occur.

\section{NOVICE}

Novice-level speakers can communicate short messages on highly predictable, everyday topics that affect them directly. They do so primarily through the use of isolated words and phrases that have been encountered, memorized, and 
recalled. Novice-level speakers may be difficult to understand even by the most sympathetic interlocutors accustomed to non-native speech.

\section{Novice High}

Speakers at the Novice High sublevel are able to handle a variety of tasks pertaining to the Intermediate level, but are unable to sustain performance at that level. They are able to manage successfully a number of uncomplicated communicative tasks in straightforward social situations. Conversation is restricted to a few of the predictable topics necessary for survival in the target language culture, such as basic personal information, basic objects, and a limited number of activities, preferences, and immediate needs. Novice High speakers respond to simple, direct questions or requests for information. They are also able to ask a few formulaic questions.

Novice High speakers are able to express personal meaning by relying heavily on learned phrases or recombinations of these and what they hear from their interlocutor. Their language consists primarily of short and sometimes incomplete sentences in the present, and may be hesitant or inaccurate. On the other hand, since their language often consists of expansions of learned material and stock phrases, they may sometimes sound surprisingly fluent and accurate. Pronunciation, vocabulary, and syntax may be strongly influenced by the first language. Frequent misunderstandings may arise but, with repetition or rephrasing, Novice High speakers can generally be understood by sympathetic interlocutors used to non- natives. When called on to handle a variety of topics and perform functions pertaining to the Intermediate level, a Novice High speaker can sometimes respond in intelligible sentences, but will not be able to sustain sentence-level discourse.

Source: ACTFL (American Council on the Teaching of Foreign Languages) Retrieved on 03/12/14 from:

http://www.actfl.org/sites/default/files/pdfs/ACTFLProficiencyGuidelines2012-Speaking.pdf 


\section{Appendix E}

Graphs of social presence score for Jennifer (Café and Individual Room)

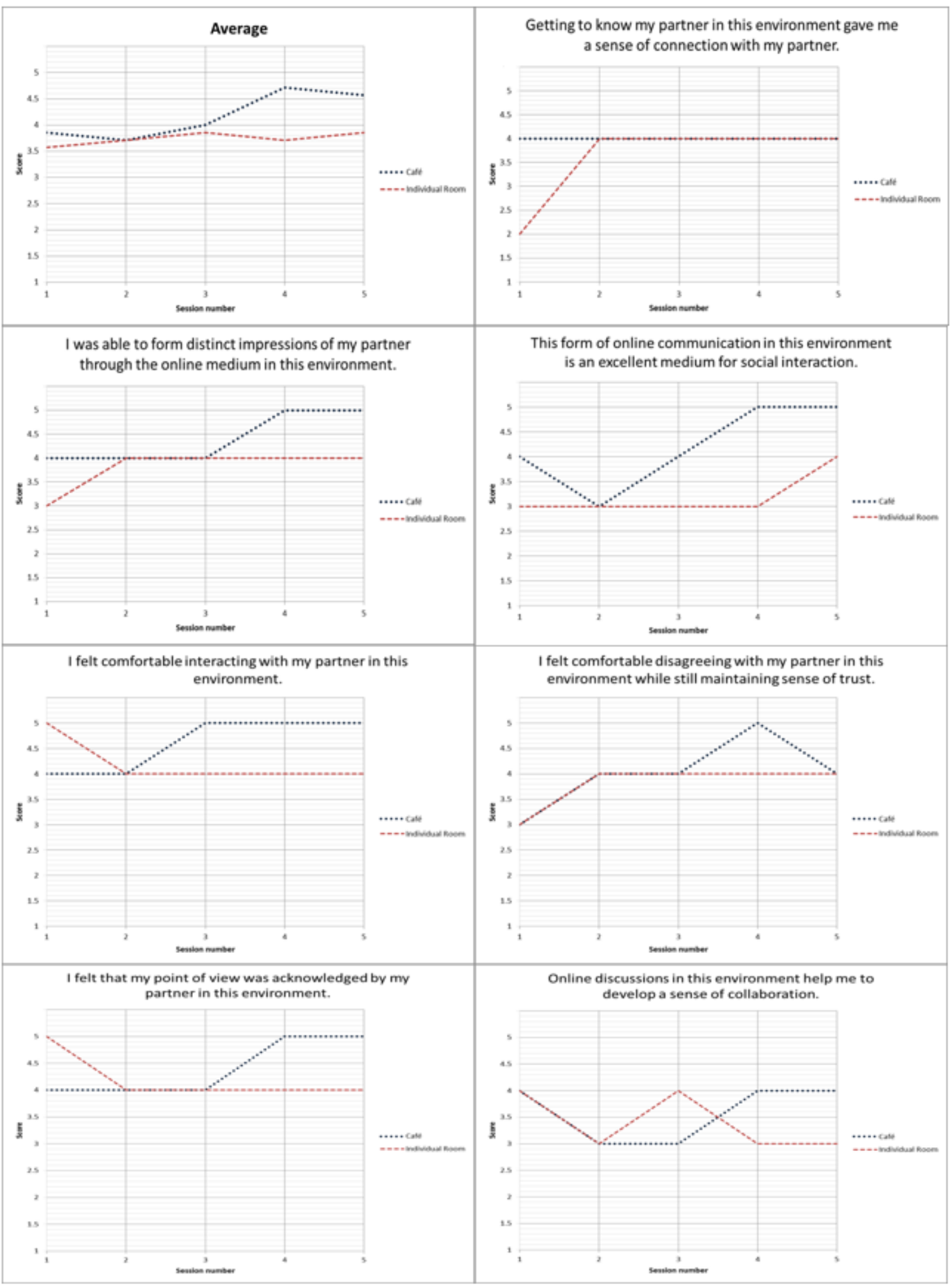


Appendix F

Graphs of social presence score for Mary (Café and Individual Room)

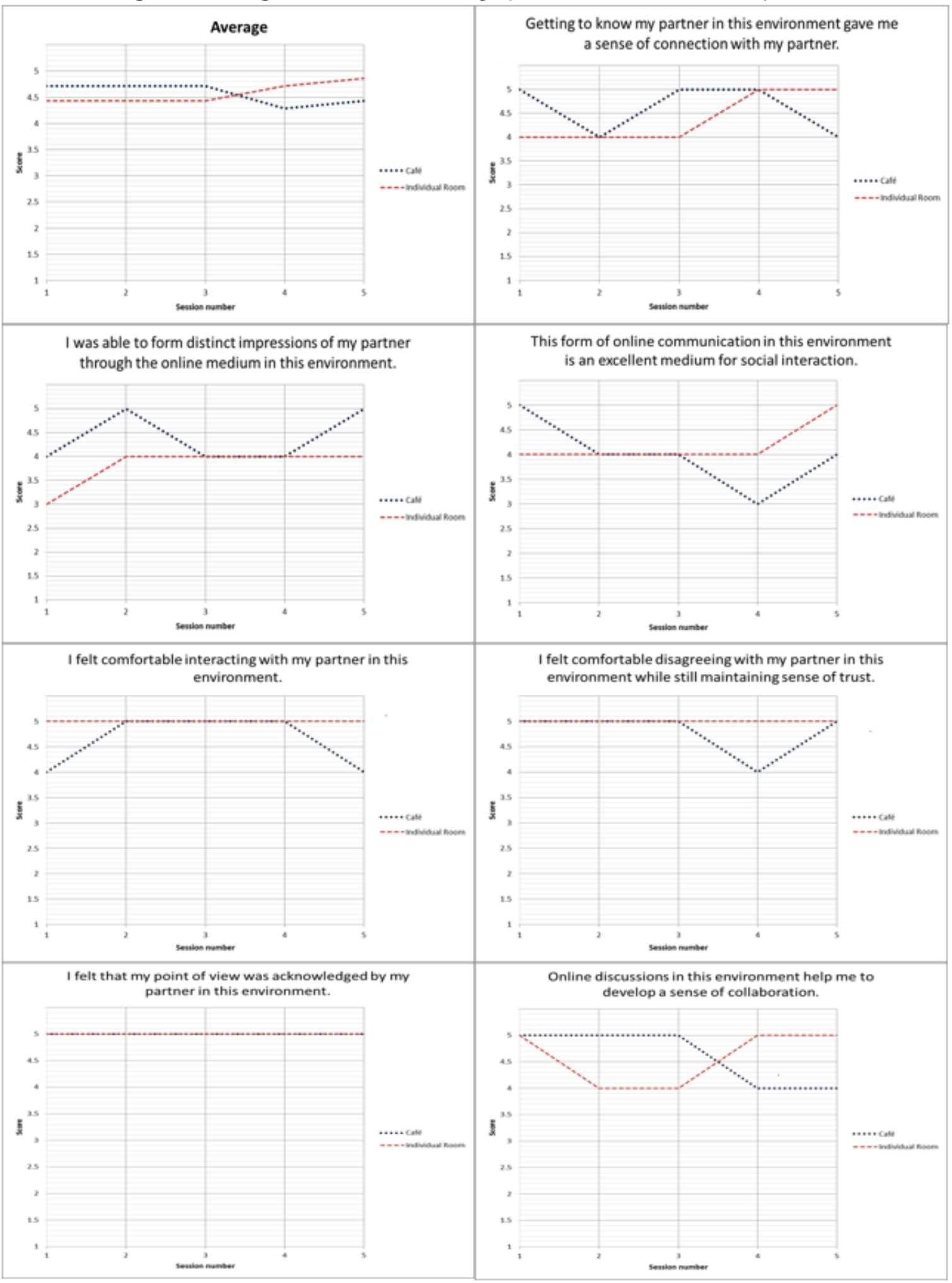


Appendix G

Graphs of social presence score for Yoko (Café and Individual Room)

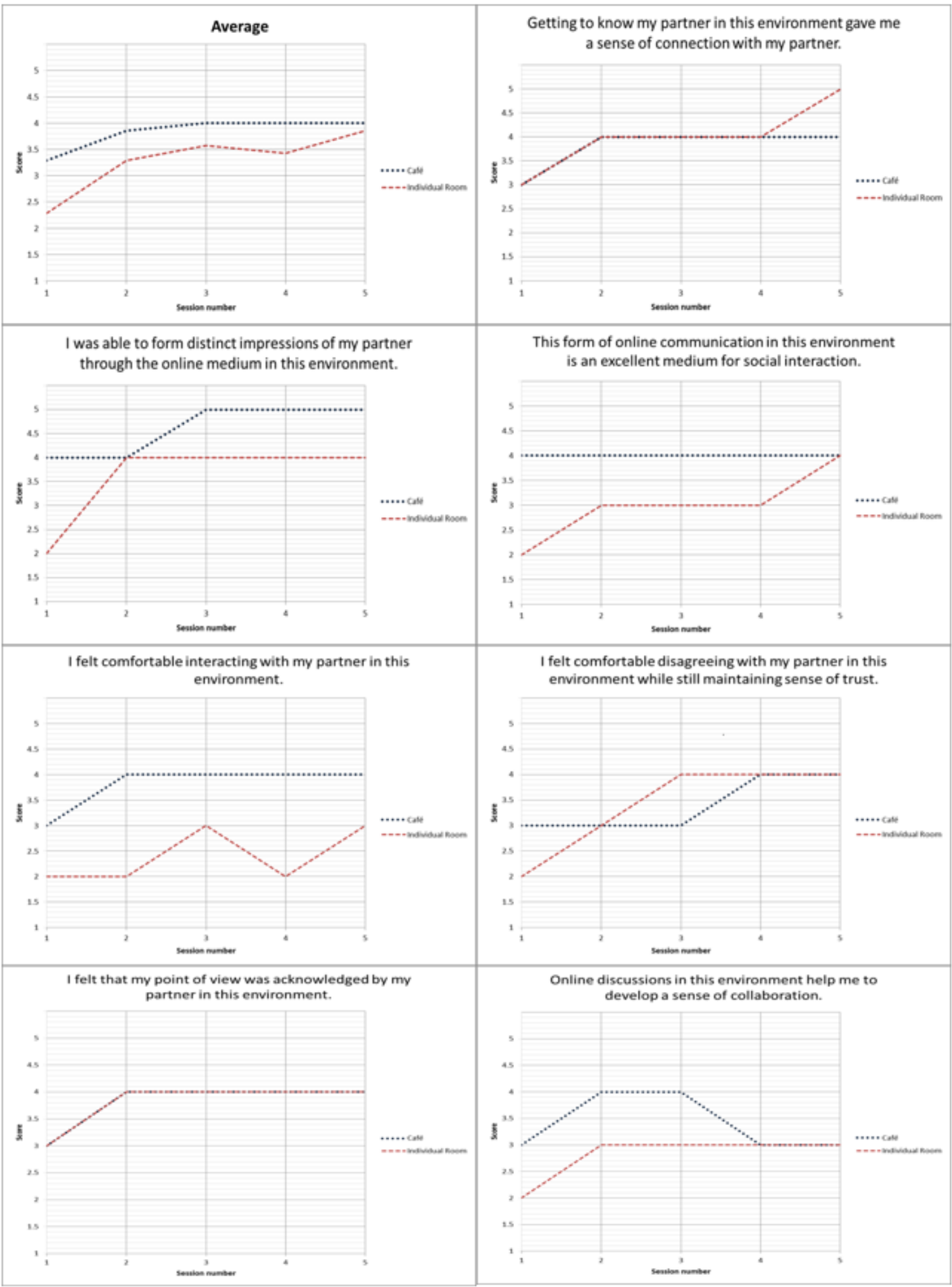


Appendix $\mathrm{H}$

Graphs of social presence score for Keiko (Café and Individual Room)

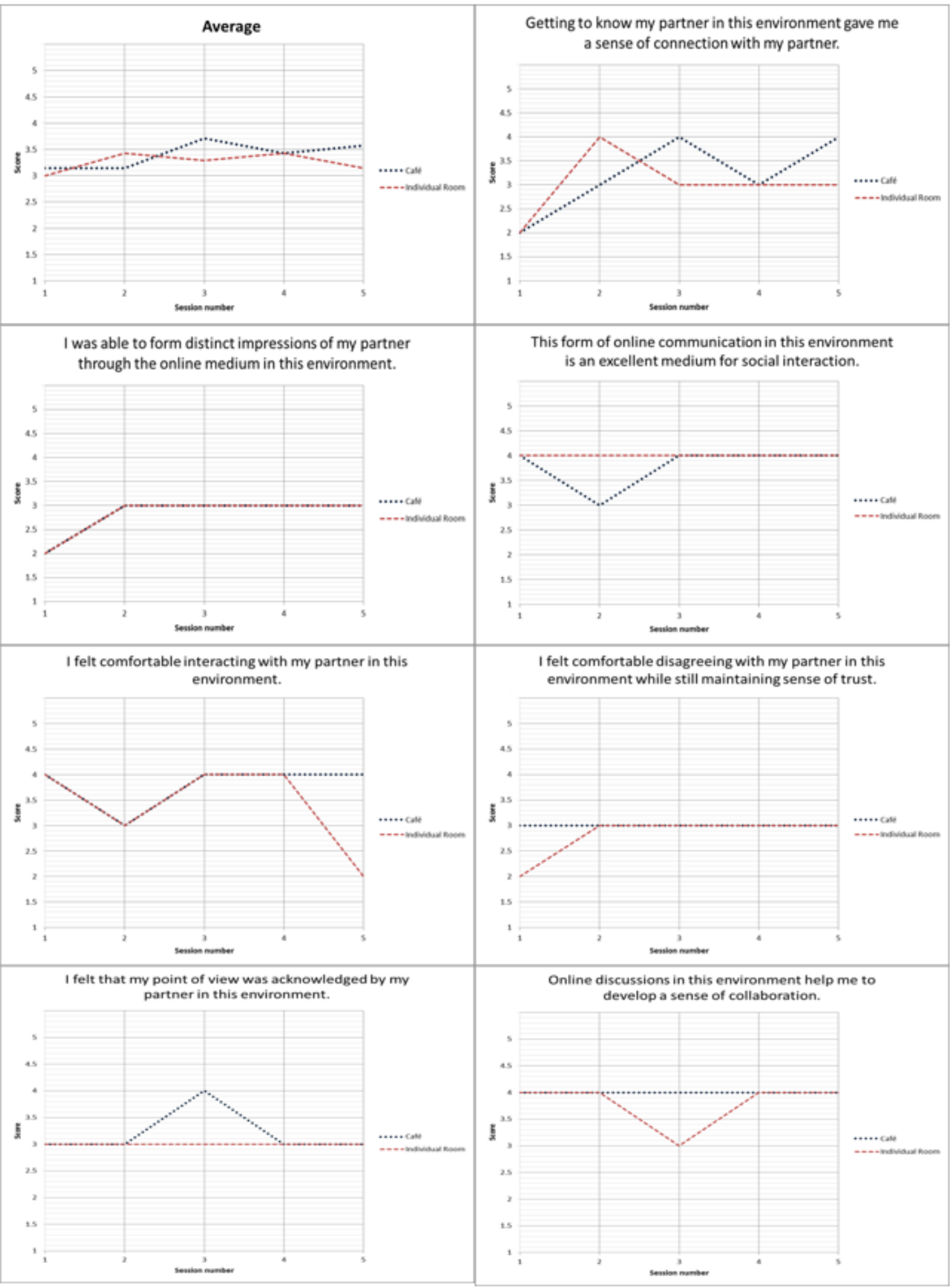




\section{Appendix I}

Graphs of social presence score for Jennifer and Yoko (Individual Room)

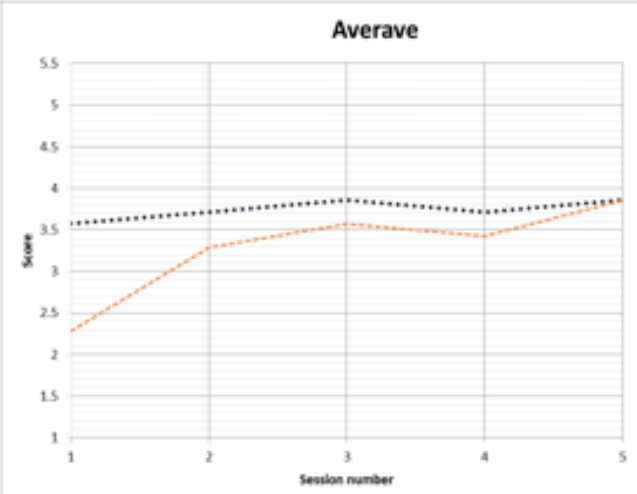

I was able to form distinct impressions of my partner through the online medium in this environment.

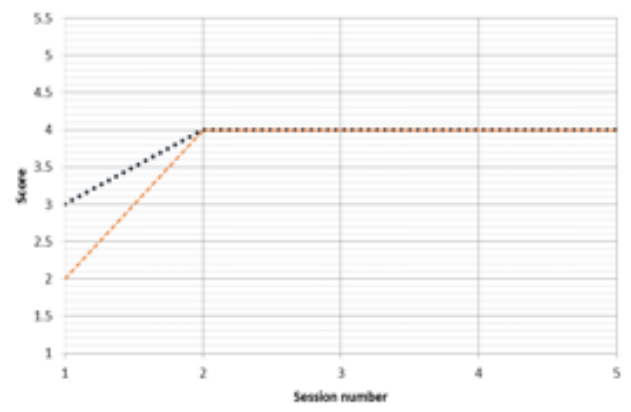

I felt comfortable interacting with my partner in this environment.

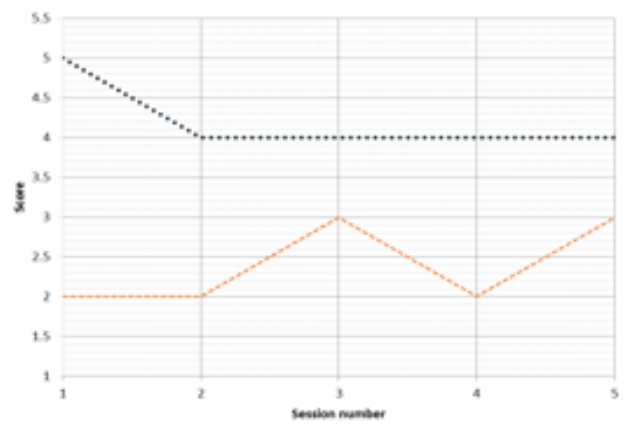

I felt that my point of view was acknowledged by my partner in this environment.

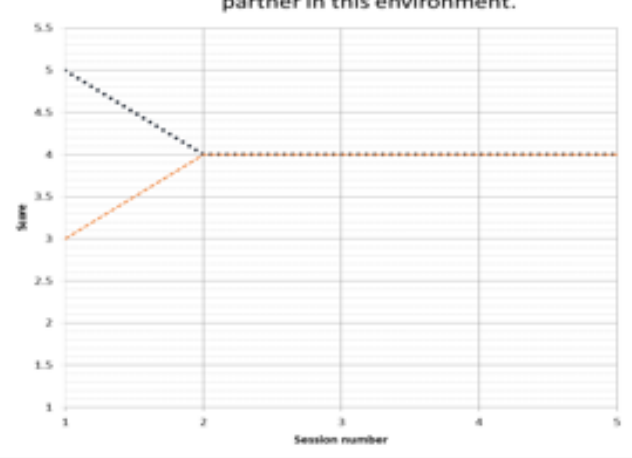

Getting to know my partner in this environment gave me a sense of connection with my partner.

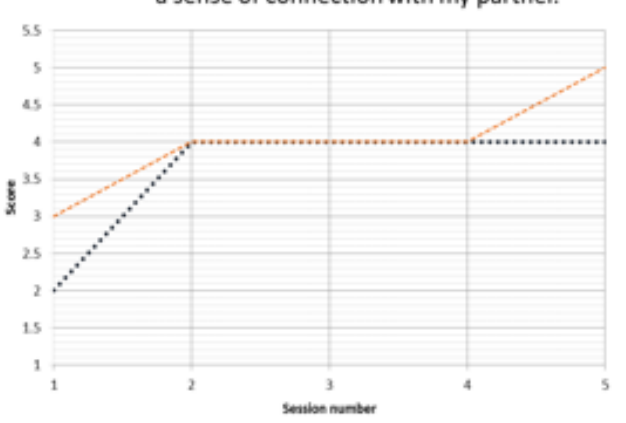

This form of online communication in this environment is an excellent medium for social interaction.

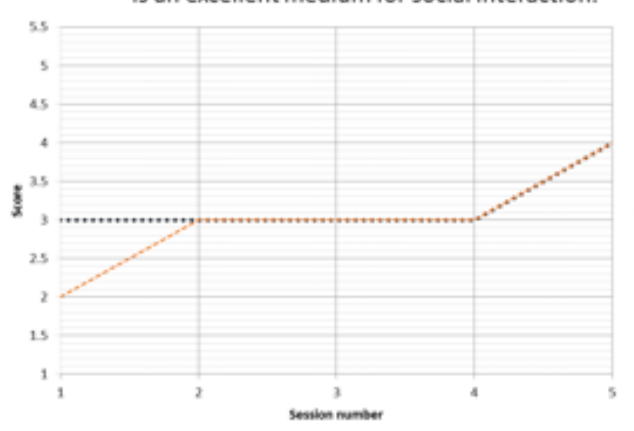

I felt comfortable disagreeing with my partner in this environment while still maintaining sense of trust.
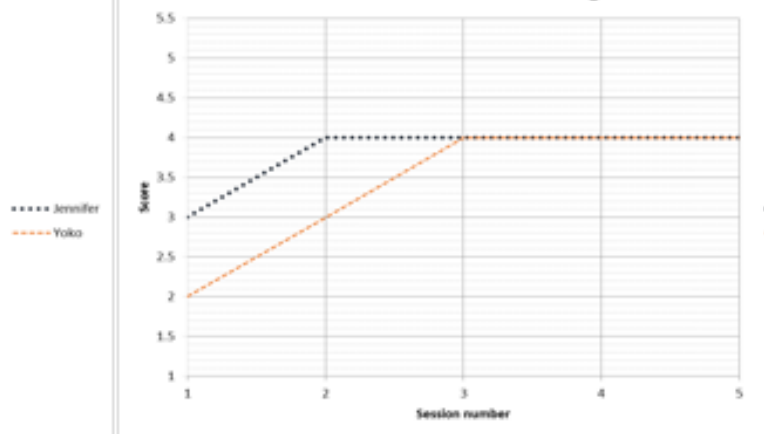

Online discussions in this environment help me to develop a sense of collaboration.
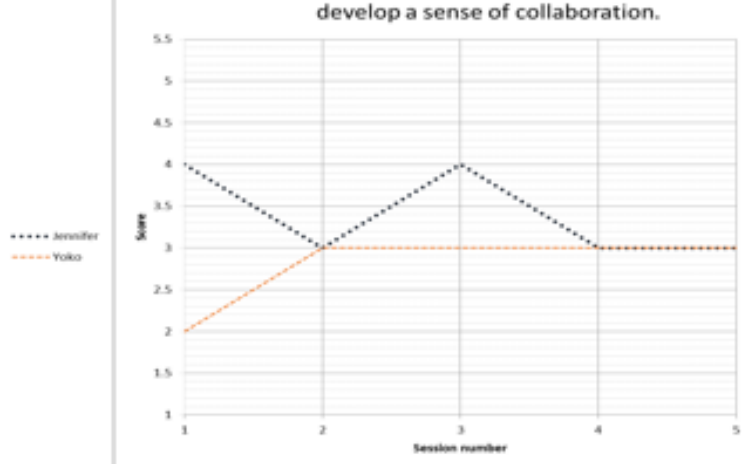
Appendix J

Graphs of social presence score for Jennifer and Keiko (Café)

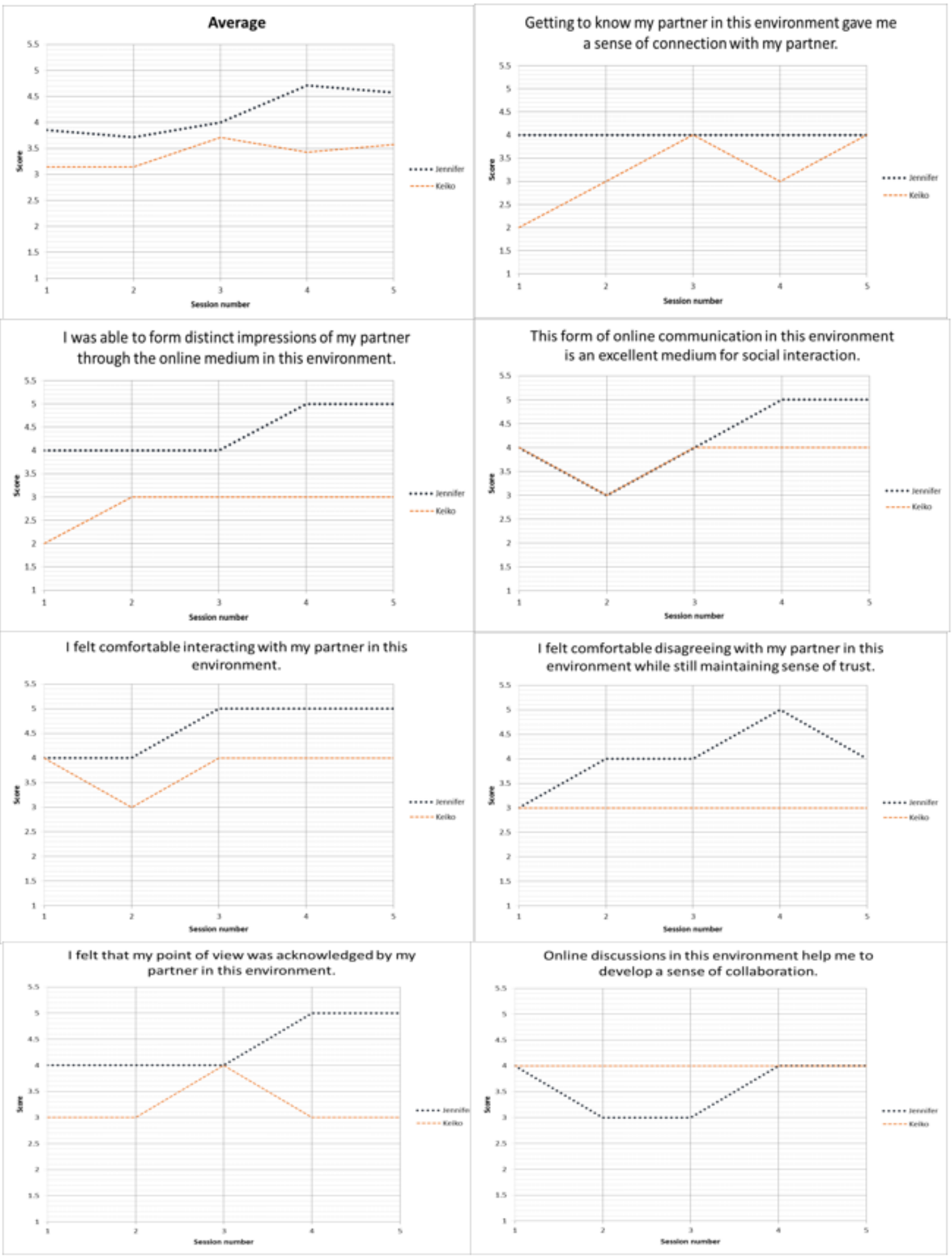




\section{Appendix K}

Graphs of social presence score for Mary and Yoko (Café)

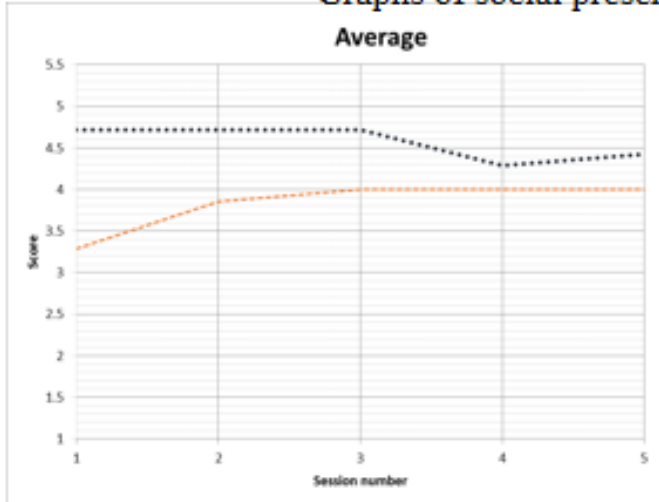

I was able to form distinct impressions of my partner through the online medium in this environment.
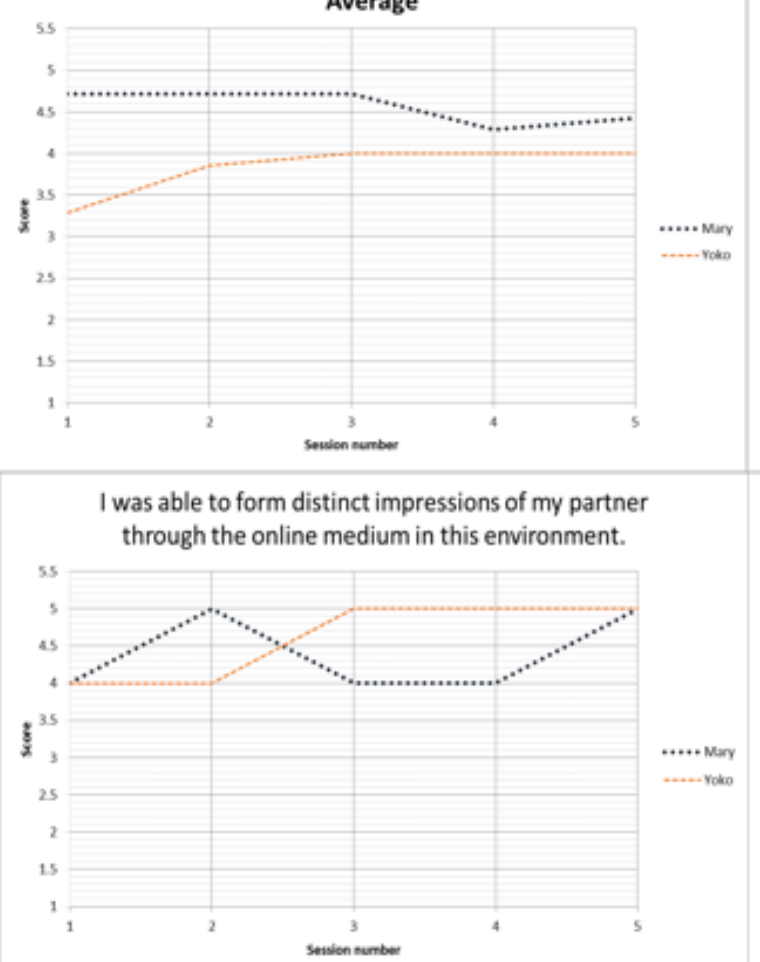

Getting to know my partner in this environment gave me a sense of connection with my partner.

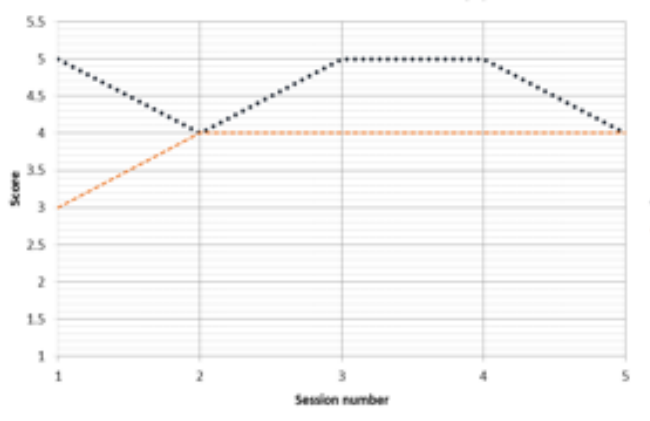

This form of online communication in this environment is an excellent medium for social interaction.

I felt comfortable interacting with my partner in this environment.
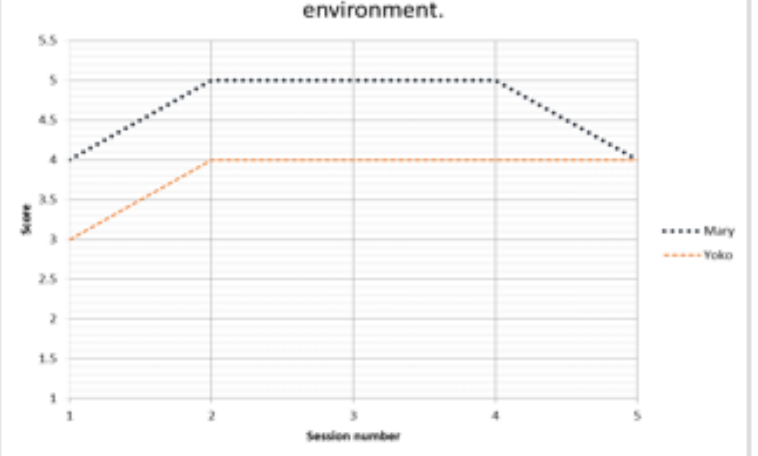
environment while still maintaining sense of trust.

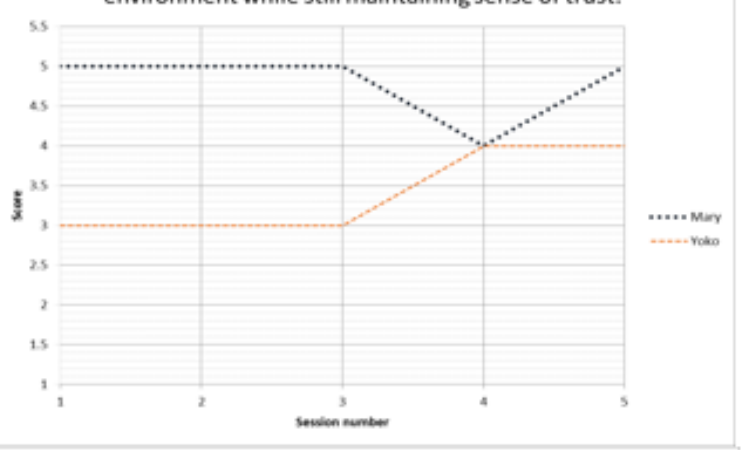

I felt that my point of view was acknowledged by my partner in this environment.

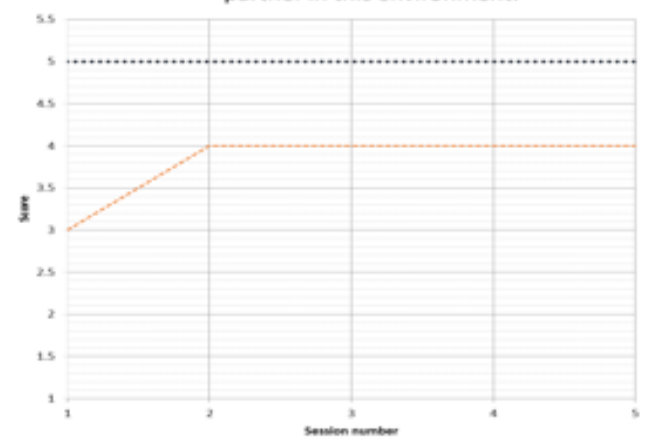

Online discussions in this environment help me to develop a sense of collaboration.

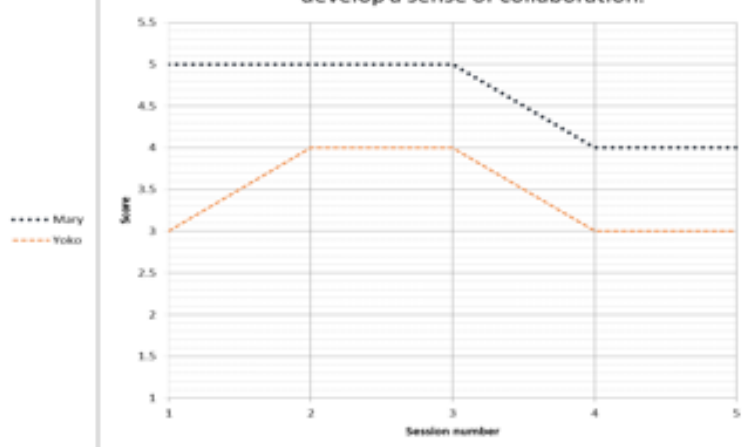


Appendix L

Graphs of social presence score for Mary and Keiko (Individual Room)
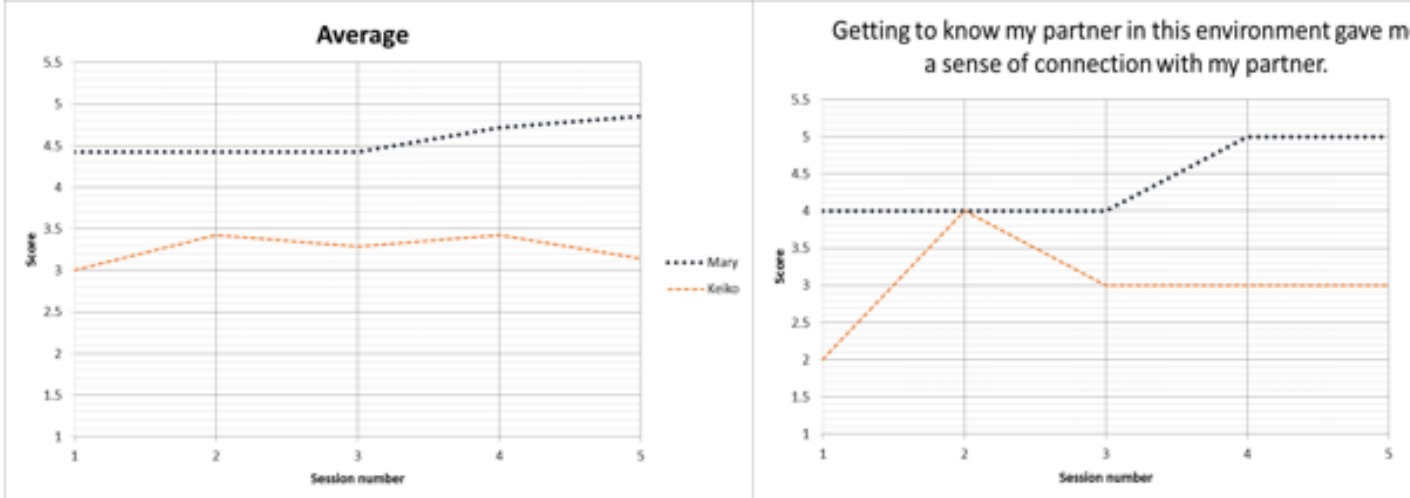
sense of connection with my partner.

I was able to form distinct impressions of my partner through the online medium in this environment.
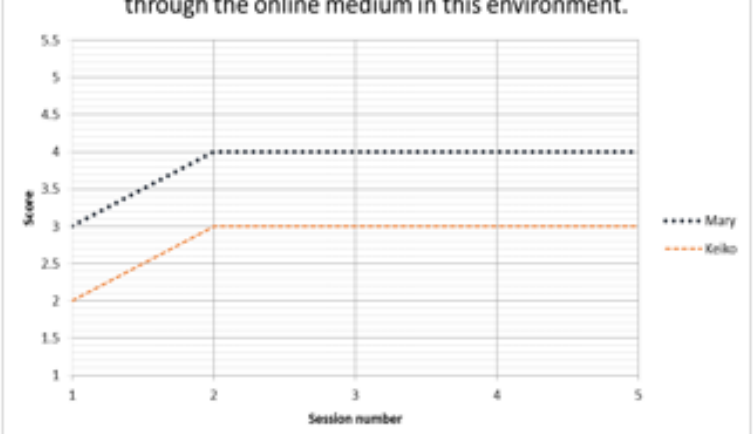

This form of online communication in this environment is an excellent medium for social interaction.

I felt comfortable interacting with my partner in this environment.
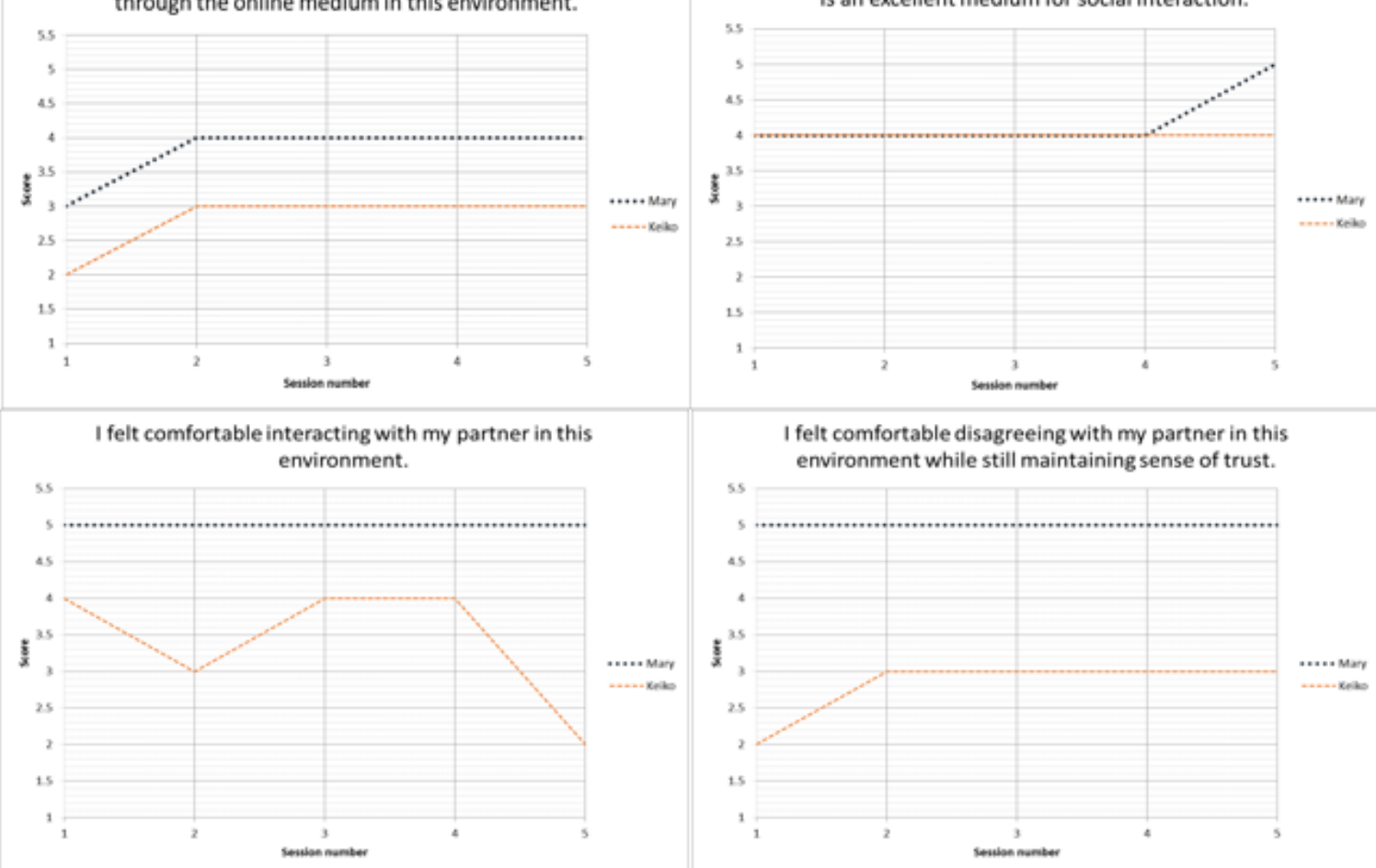

I feit that my point of view was acknowledged by my partner in this environment.
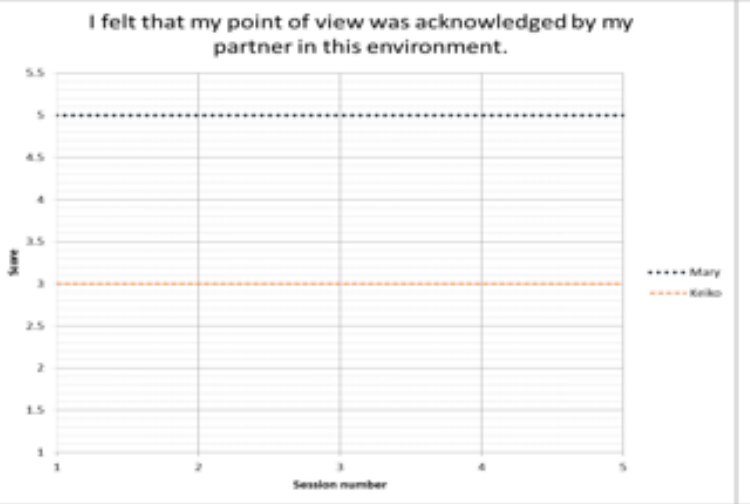

I felt comfortable disagreeing with my partner in this environment while still maintaining sense of trust.
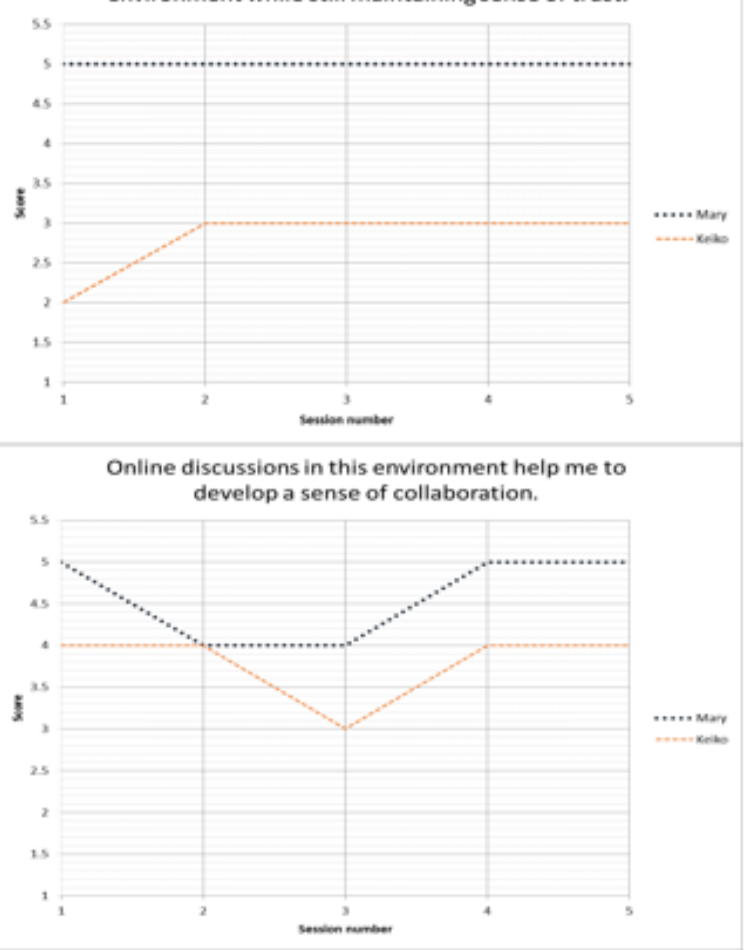


\section{Appendix M}

Graph for the number of AS-unit and self-disclosure for Jennifer (Café and Individual Room)

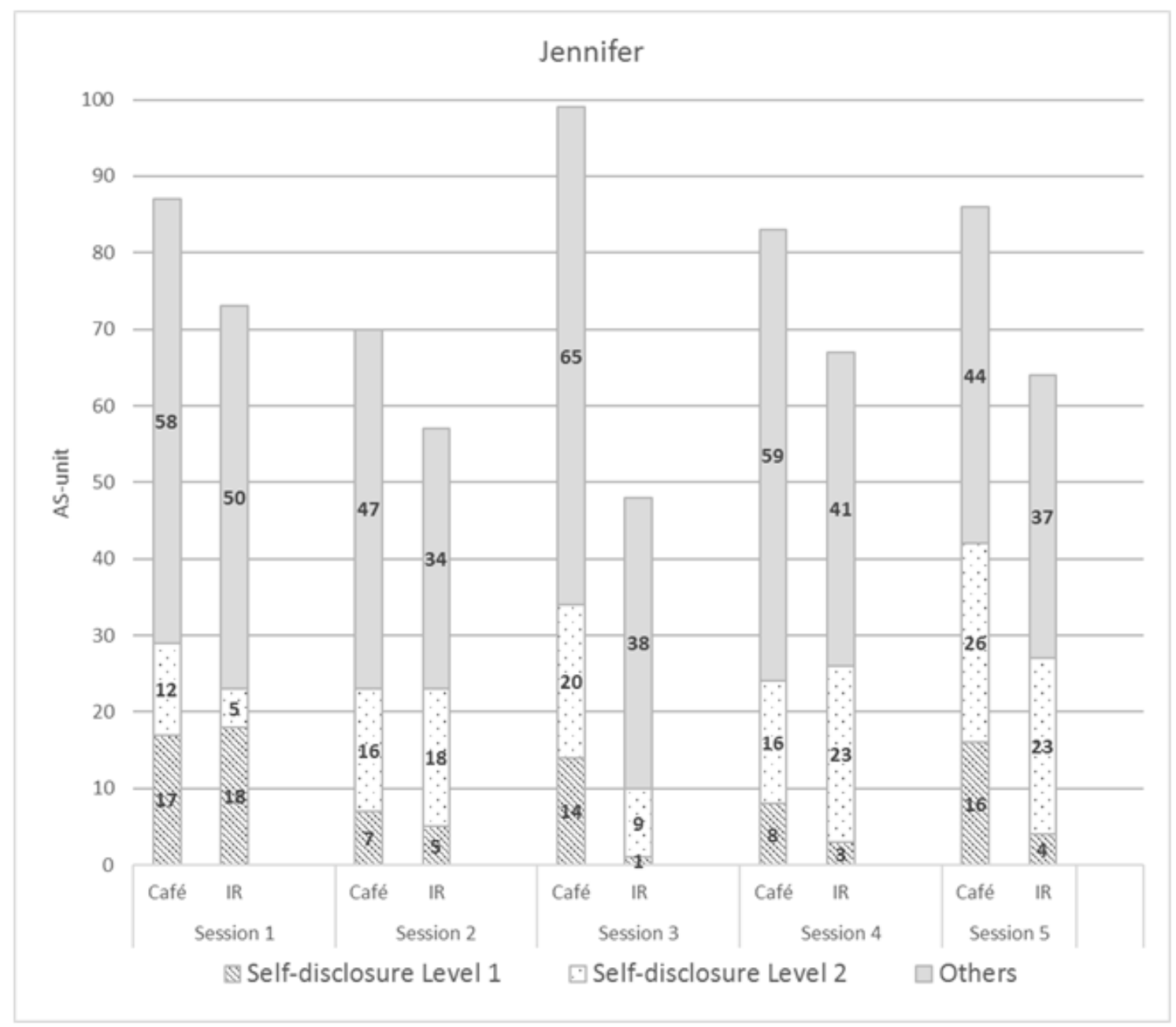




\section{Appendix N}

Graph for the number of AS-unit and self-disclosure for Mary (Café and Individual Room)

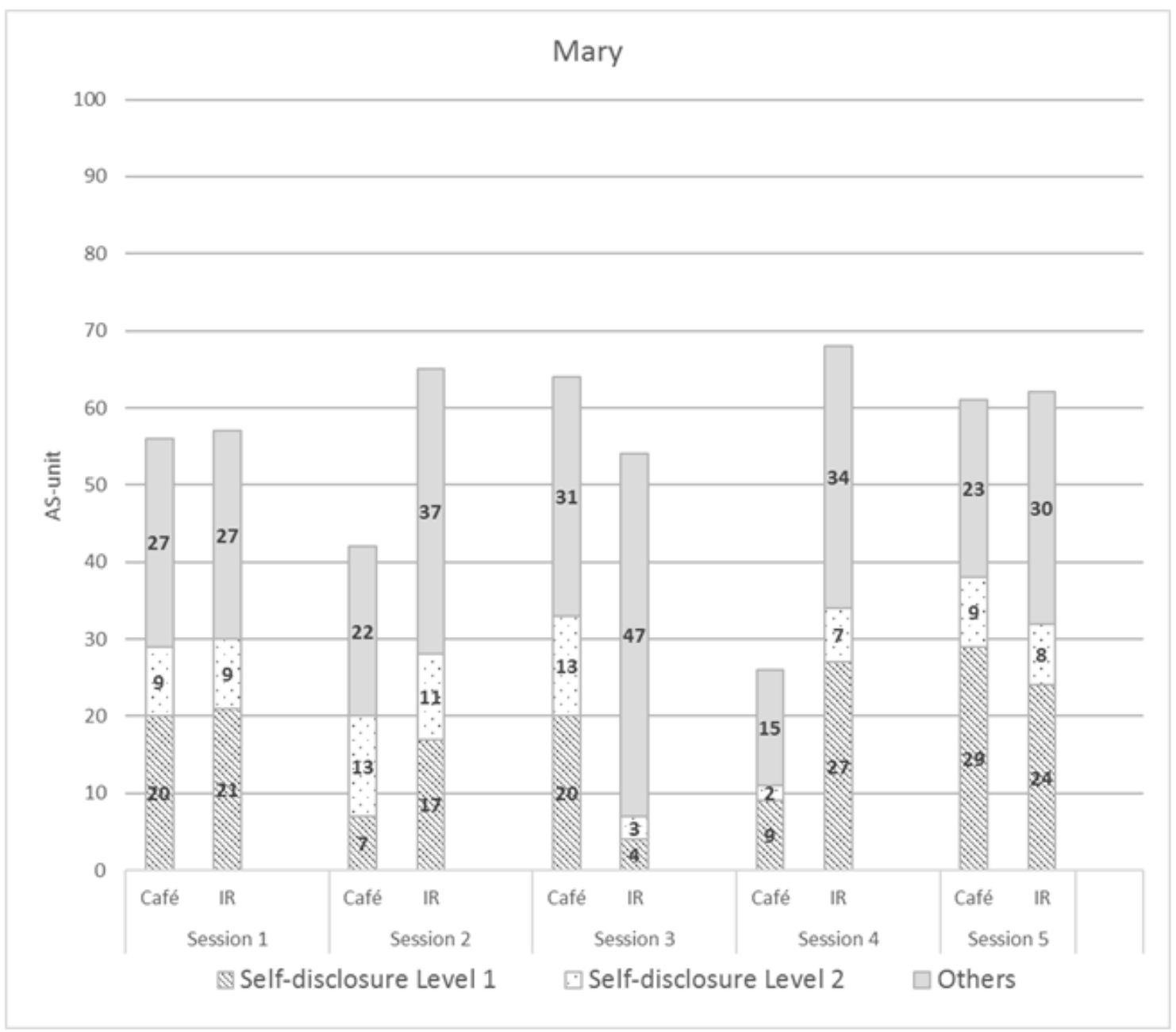




\section{Appendix O}

Graph for the number of AS-unit and self-disclosure for Yoko (Café and Individual Room)

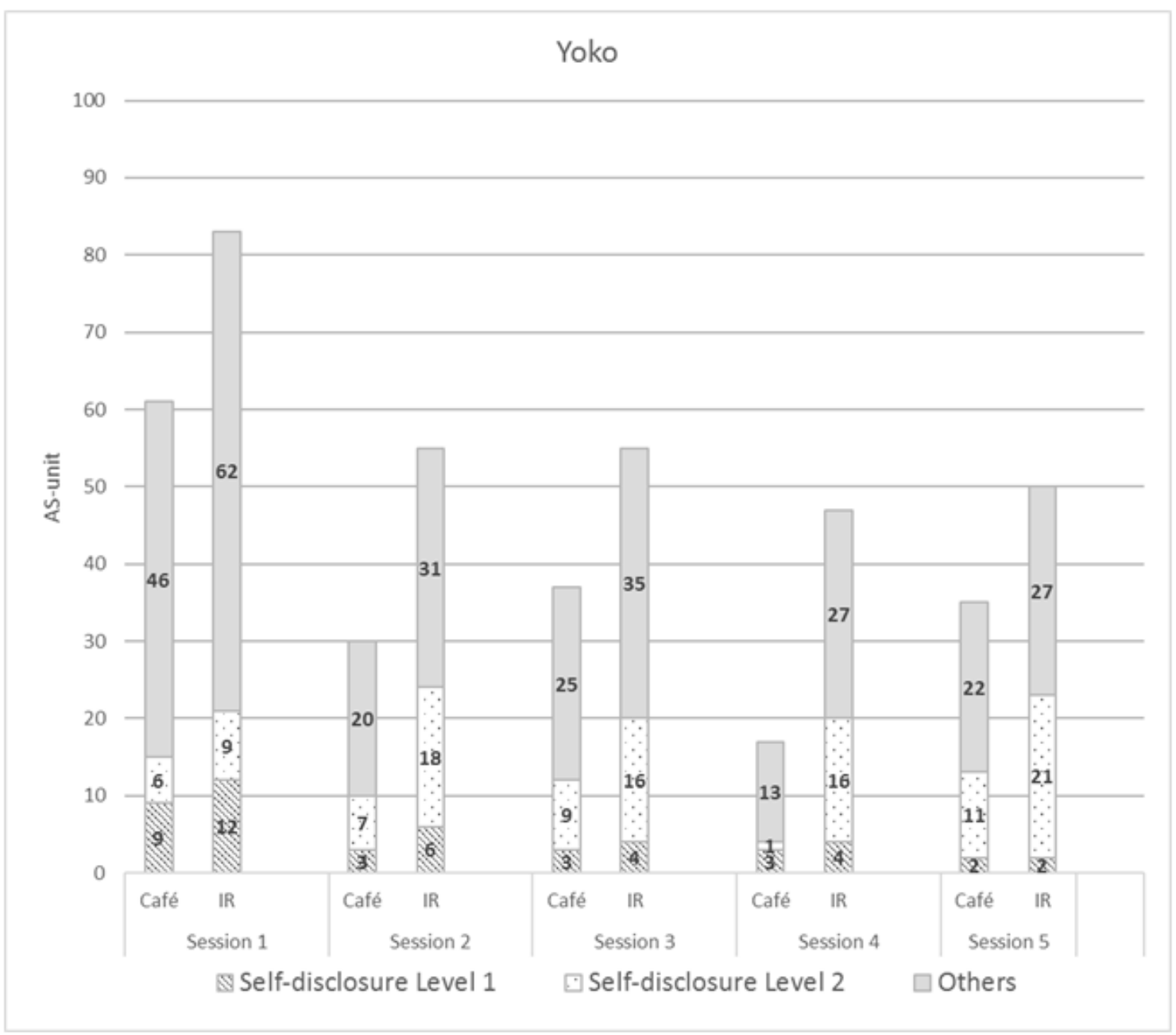


Appendix P

Graph for the number of AS-unit and self-disclosure for Keiko (Café and Individual Room)

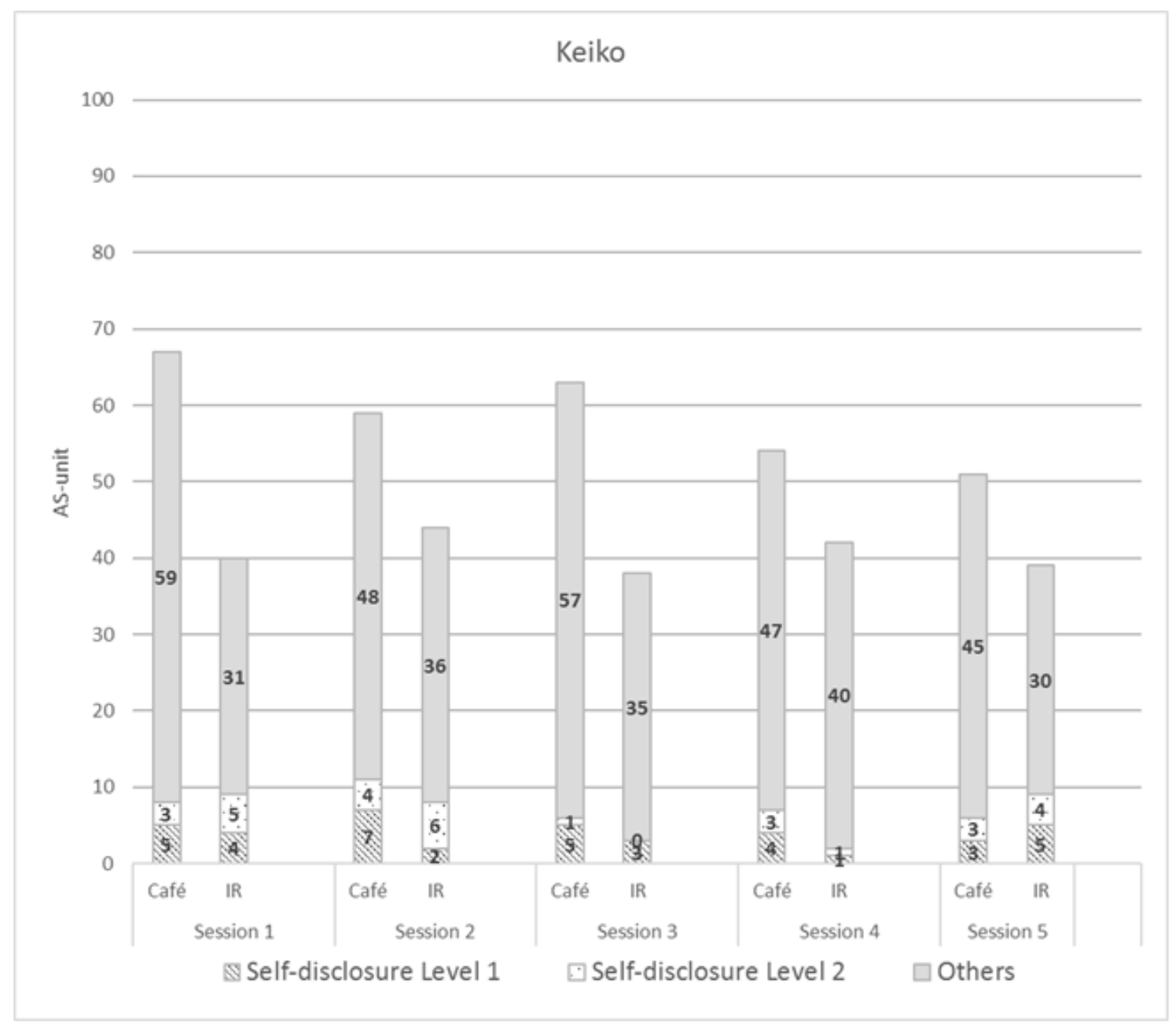




\section{Appendix Q}

Graph for the number of AS-unit and self-disclosure for Jennifer and Yoko (Individual Room)

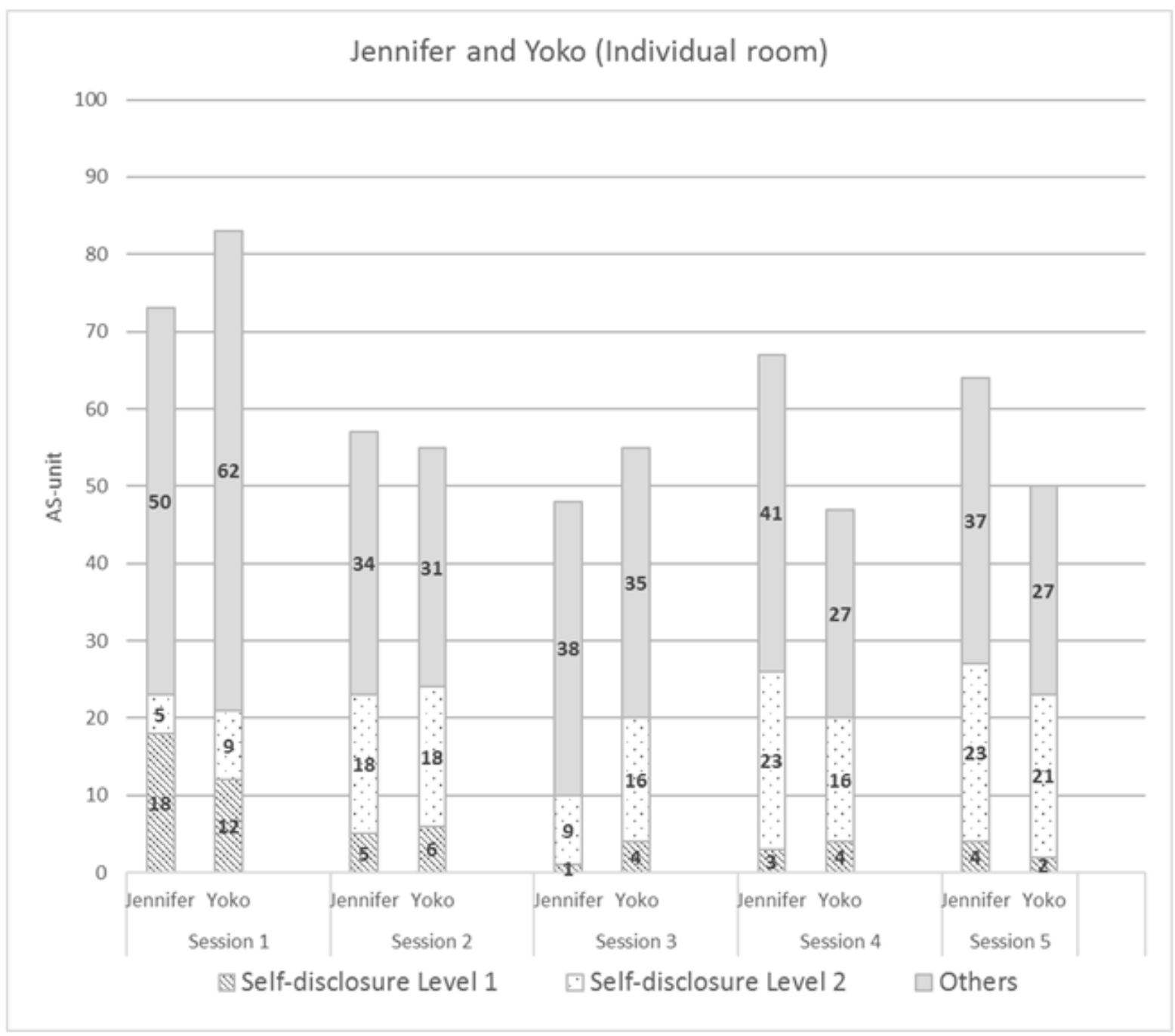




\section{Appendix R \\ Graph for the number of AS-unit and self-disclosure for Jennifer and Keiko (Café)}

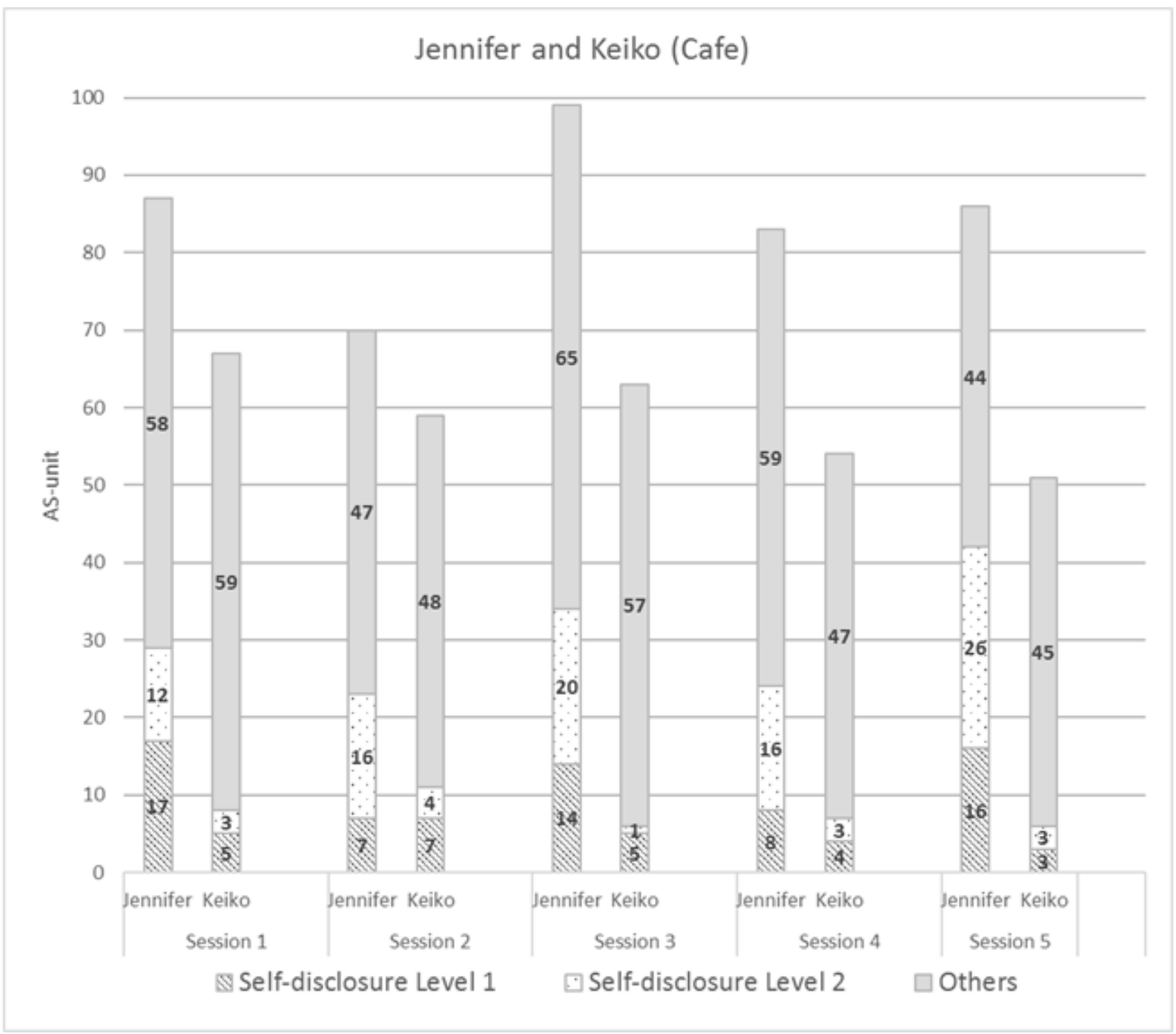




\section{Appendix S}

Graph for the number of AS-unit and self-disclosure for Mary and Yoko (Cafe)

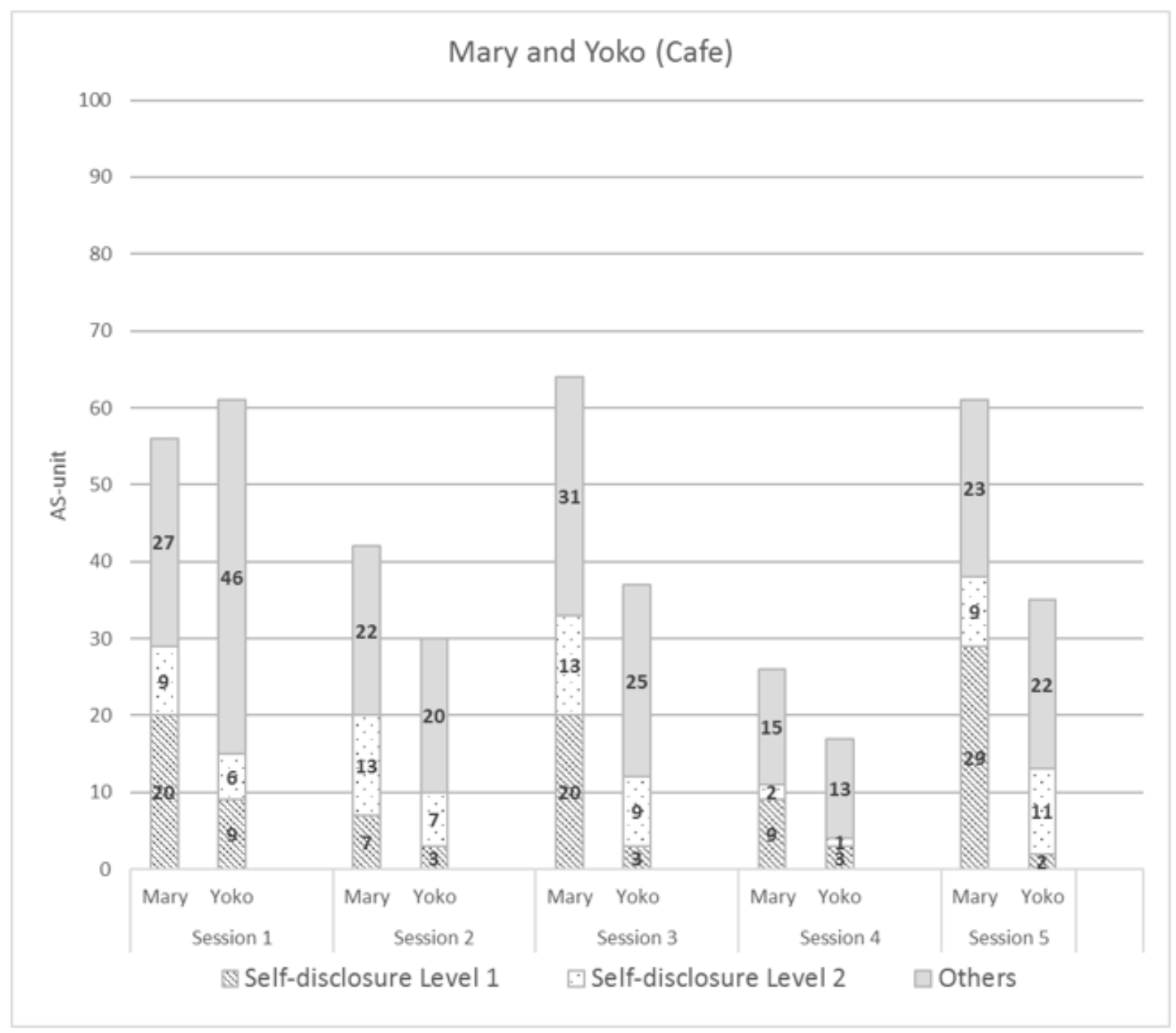




\section{Appendix T}

Graph for the number of AS-unit and self-disclosure for Mary and Keiko (Individual Room)

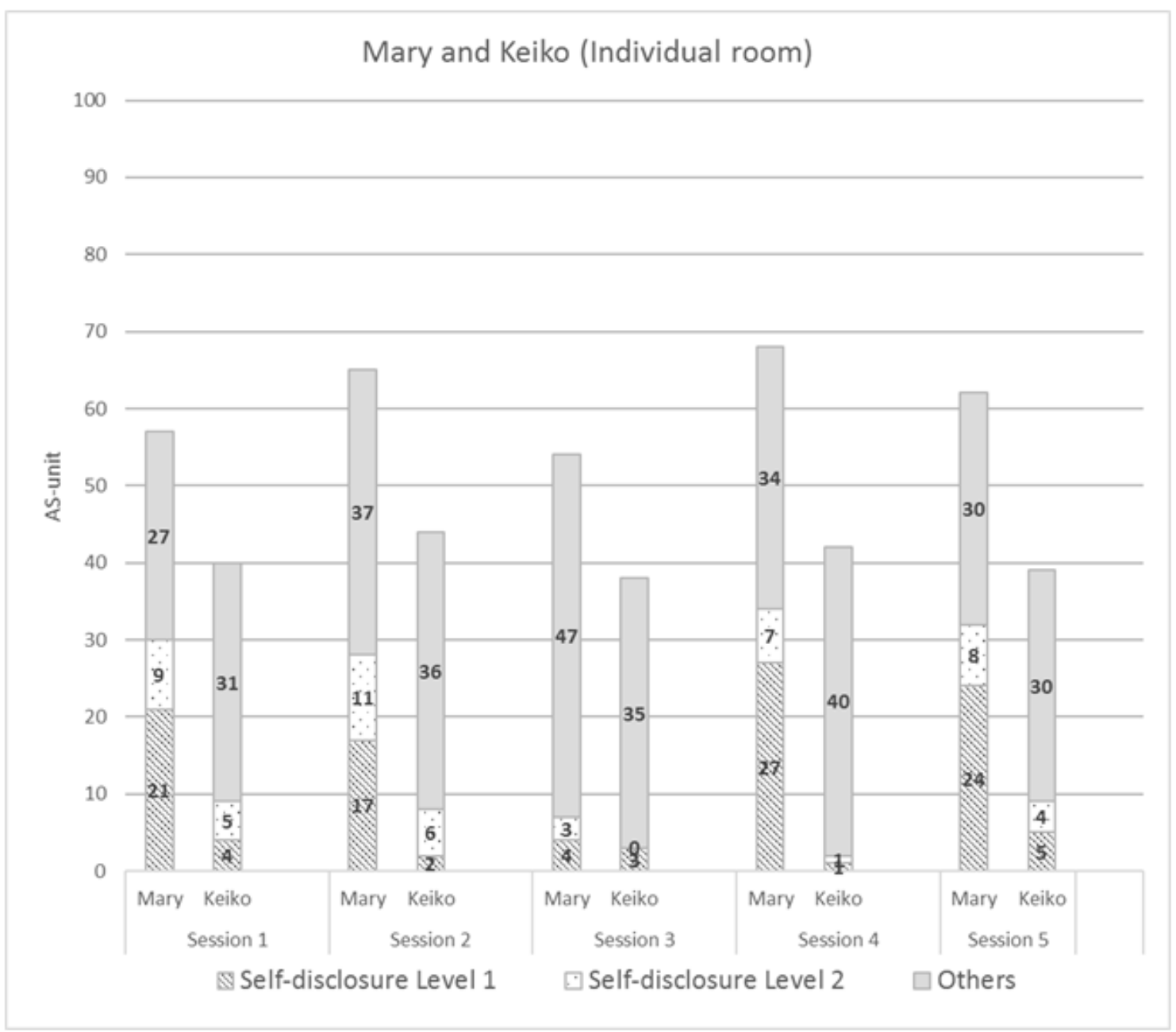




\title{
Appendix U
}

\author{
Notice of Approval from IRB
}

Princeton University

Institutional Review Board For Human Subjects, Office of Research Integrity and Assurance

\section{NOTICE OF APPROVAL}

To: Hisae Matsui

Date: December 04, 2013

RE: $\quad$ Protocol \#: 0000005426

Protocol Title: The Impact of Visual Elements in Virtual Environments on Interpersonal Emotional Connection in Telecollaboration

\author{
Approval Date: November 23, 2013 \\ Expiration Date: $\quad$ November 22, 2014 \\ Type of Review: $\quad$ Expedited Category: 7 \\ Risk Level: Minimal Risk \\ Submission Type: Continuing Review \# 2 with Amendment- Research purpose was slightly modified; Questions in \\ Questionnaire were modified; Conversation analysis will be conducted to analyze recorded data; \\ Study population was changed to students in JPN105 and JPN301; Study title changed.
}

1. Adverse Events: Any unanticipated problems involving risk to subjects or others that occurs in relation to this study must be reported to the IRB Office within 10 days.

2. Continuing Review: It is the Principal Investigator's responsibility to obtain review and continued approval before expiration date shown above. You may not continue any research activity beyond the expiration date without IRB approval. The IRB must review and approve all human subject research studies that are not exempt at intervals appropriate to the degree of risk, at least once per year, as required by 45 CFR 46 .

--In order to avoid lapses in approval of your research and automatic suspension of subject enrollment, please submit a completed Continuation Form and all current documents being used at least six weeks before the study expiration date.

-Failure to receive continuation approval before the expiration date will result in the automatic suspension of the approval of this protocol on the expiration date.

3. Changes/Amendments to Approved Research:All changes or amendments to your protocol, consent form(s) or any other aspect of this study require review and approval by the IRB BEFORE implementation.

4. Completion of Study: If the research, including data analysis has been completed or you wish to terminate the study for any reason, please notify the IRB via email at irb@princeton.edu and include in the written notification, the reason for study termination.

5. This approval does not replace any departmental or other approvals that may be required.

Mailing Address: RIA | Princeton University | Princeton, NJ 08544 Located at: 87 Prospect Ave. 3rd Floor | irb@princeton.edu 


\section{Appendix V \\ STANDARD ADULT CONSENT FORM \\ PRINCETON UNIVERSITY \\ [IRB \# 5426]}

TITLE OF RESEARCH: The Impact of Visual Elements in Virtual Environments on Interpersonal Emotional Connection in Telecollaboration

研究のタイトル：仮想環境の視覚的要素がテレコラボレーションの中で他者との感情の つながりに与える影響

INVESTIGATOR: Hisae Matsui

研究者 : 松井久恵

The following informed consent is required by Princeton University for any research study conducted by investigators at the University. This study has been approved by the University's Institutional Review Board for Human Subjects.

以下の同意書はプリンストン大学からの要請で、大学の研究者によって行われる調査研 究における全ての関係者が同意し、署名する事が必要とされているものです。この研究 は大学内被験者のための倫理委員会によって承認されました。

\section{Purpose of Research:}

The purpose of this study is to examine how the participant perceives the usefulness of the virtual elements in 3D virtual environments to develop an interpersonal emotional connection with his/her partner in telecollaboration. You are being asked to participate because you are taking Intermediate Japanese 1 (JPN105) or Advanced Japanese 1 (JPN301).

この研究の目的は、3D 仮想環境上での視覚的要素の他者との感情のつながりの発展に 与える有効性がテレコラボレーションの中で被験者にどのように知覚されるかを調査す るものです。あなたは異文化コミュニケーションのゼミ生であるためにこの研究への参 加を頼まれました。

\section{Procedures:}

You will be asked to have conversation sessions with your partner in Second Life and answer survey questionnaires after each session as well as at the end of all sessions. I expect your participation to take about 30 minutes of your time for each session and for answering survey questionnaires.

あなたはセカンドライフ内でパートナーと会話のセッションを持ち、その後各セッショ ン後と全てのセッション後にアンケートに答えることを頼まれます。各セッションとア ンケートの記入はおよそ 30 分程度です。

\section{Confidentiality:}

Your answers will be confidential. The records collected during this study will be kept private. I will not include any information that will make it possible to identify you. Research records will be kept in a locked file and only the researcher will have access to your records.

あなたの答えは全て機密データとして扱われます。集められたデータは全て安全に保管 
されます。個人が特定できるデータは一緒に保管されません。研究データは施錠された ファイルに保管され、研究者しかこのデータにアクセスできません。

Risks or Discomforts/Benefits:

The potential risks associated with this study are minimal.

この研究に関わる起こりうる危険性は最小限です。

Benefits:

I expect the project to benefit you by giving opportunities to exchange opinions with native Japanese speakers. In addition, I expect this research to benefit Japanese language education by offering insights for telecollaboration by using Second Life.

この研究による利益はあなたが日本語の非母語話者と意見を交換する機会を得られるこ とです。更に、この研究によりセカンドライフを使ったテレコラボレーションに対する 見識を与えることで日本語教育にとっての利益も期待されています。

\section{I understand that:}

My participation is voluntary, and I may withdraw my consent and discontinue participation in the project at any time. My refusal to participate will not result in any penalty or have an impact on my grades.

私はこの参加が自発的であり、いつでもこのプロジェクトへの参加を取り止める事が出 来る事を確認しました。不参加に対して如何なる罰も受けず、成績への影響がないこと も確認しています。

By signing this agreement, I do not waive any legal rights or release Princeton University, its agents, or you from liability for negligence.

私は、この協定に調印することによって、自分の法的権利を放棄せず、またプリンスト ン大学やその職員に過失責任を負う義務を免除させない事とします。

I hereby give my consent to be the subject of your research.

私は、これによりあなたの研究の対象になることに同意します。 


\section{Audio/Video Recordings:}

With your permission, I would also like to audio-record your voice and video-record your avatar in Second Life during the sessions. Please sign below if you agree to be audio and video recorded.

あなたの許可により、あなたの肉声とあなたのセカンドライフ内でのアバターの映像を 録音、録画したいと思います。もしそれに同意されるなら下に署名して下さい。

I hereby give my consent for audio and video recording:

私は、これにより録音、録画される事に同意します。 
Appendix W

Flayer for recruiting participants

JPN105/301 のみなさんへ

Second Life Project

Are you looking for opportunities for conversation practice with native Japanese speakers?

Are you interested in Japanese cultures and the way they think?

Share your thoughts with students from Waseda University in a virtual environment!

$>$ What will I be doing?

You will have conversation sessions (20 minutes each) with students from Waseda University in the Second Life, virtual environment, 10 times during the project. After each session, you will be asked to fill out a brief online survey.

Period: December, 2013 January, 2014

Session time: Twice a week, 20mintes session b/w 8PM-10PM

Location: At Matsui's office.

If you have a computer that can run Second Life program, you may have sessions at home.

Language: Japanese

Topic: Free

$>$ What are the benefits?

You will have opportunities to practice speaking and listening in Japanese regularly with native speakers of Japanese.

$>$ Purpose of this project

The main purpose of the project is to see if there is an effect of sharing environments virtually on building closeness with your partner. To find out the effect, I will set up two environments: one where you can see your partner and the other where you will be alone and cannot see him/her. You will be in both environments and experience interactions with others in those environments

Your interactions with your partners will be recorded for research purposes and the analyzed results may be published but your personal information will never be revealed. 
$>$ Interested?

Please fill out the attached form and tell me you available time. Please understand that you may not be able to participate in this project if you have limited schedule since it will make it more difficult to find your partner.

If you have questions, feel free to ask Hisae Matsui by e-mail (hmatsui@ princeton.edu) 
Appendix X

List of avatars

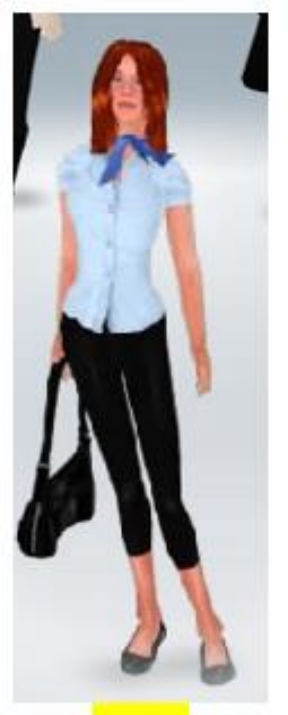

1

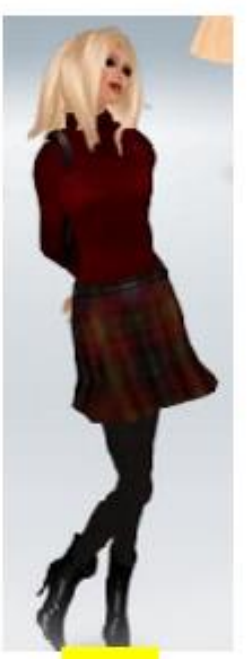

5

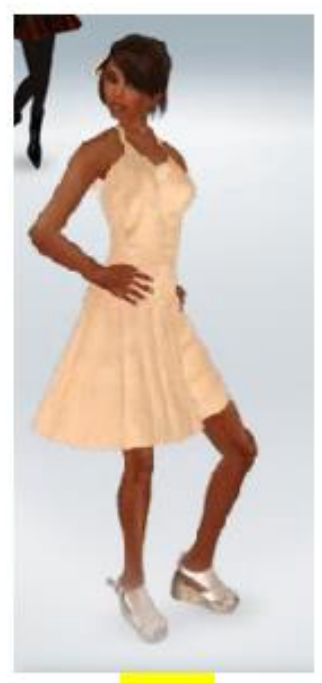

2

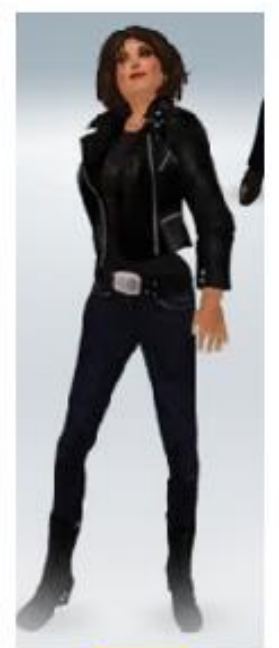

6

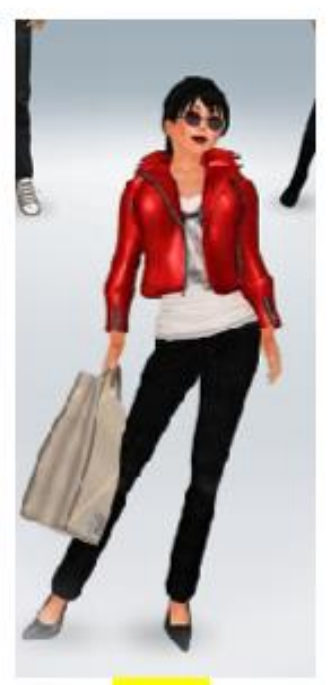

3

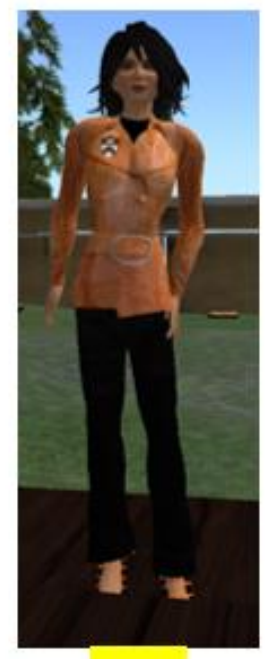

7

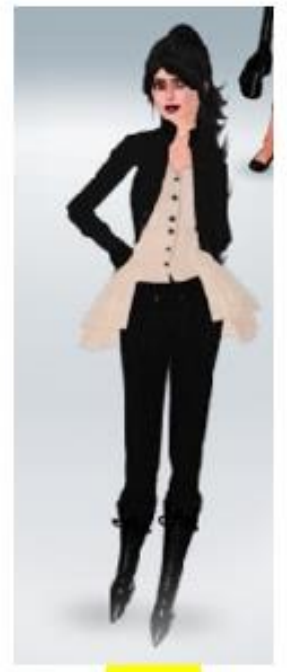

4

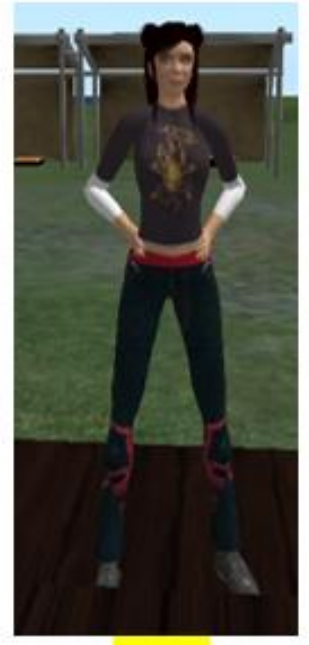

8 


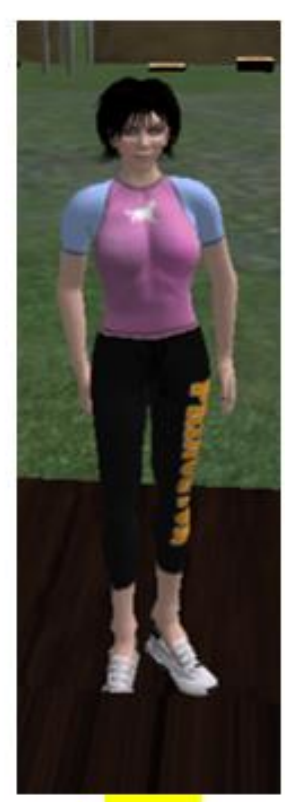

9

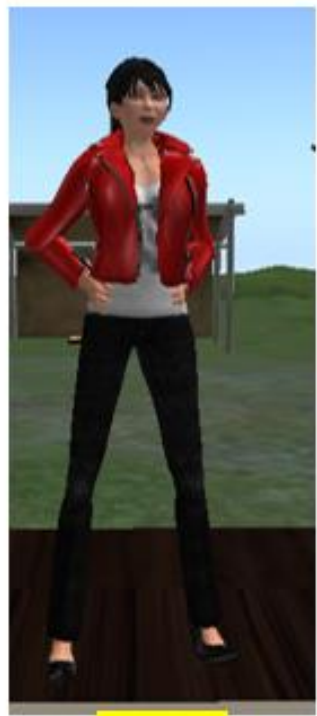

13

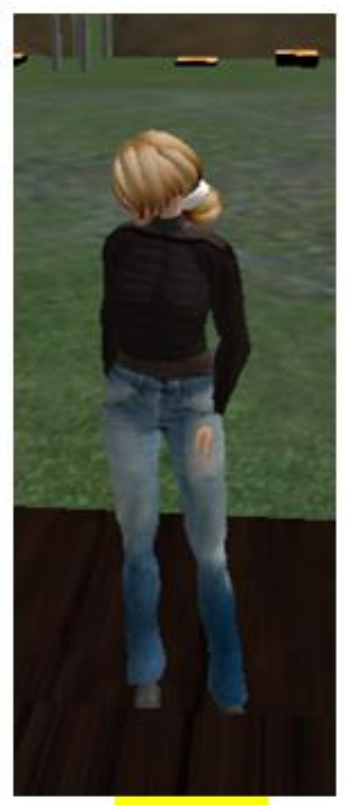

10

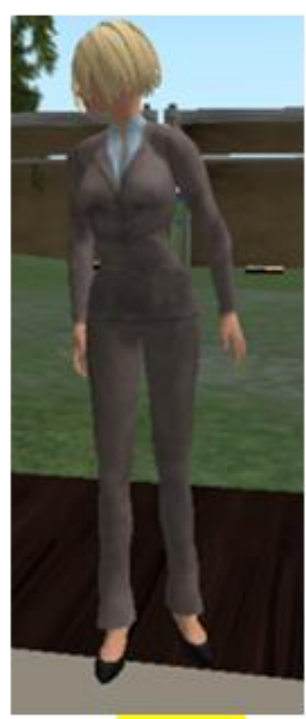

14

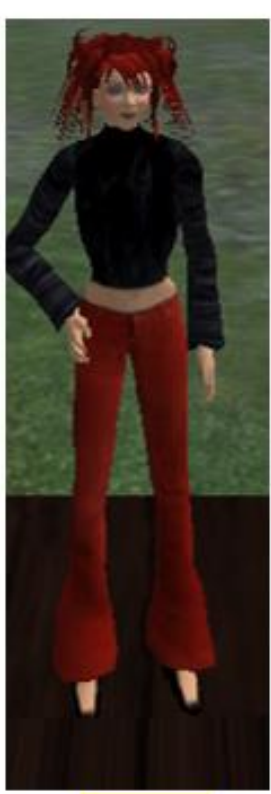

11

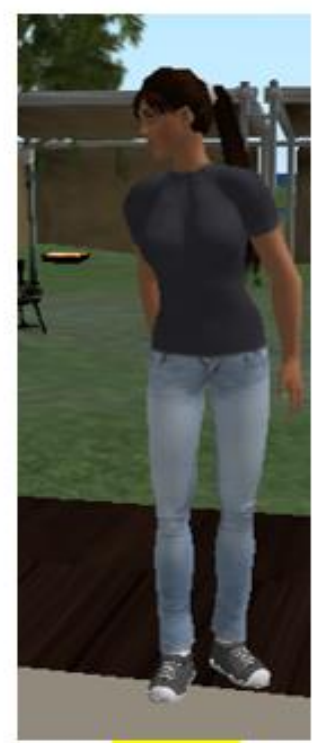

15

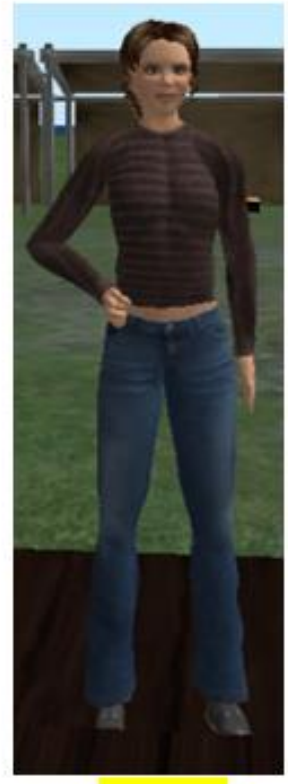

12

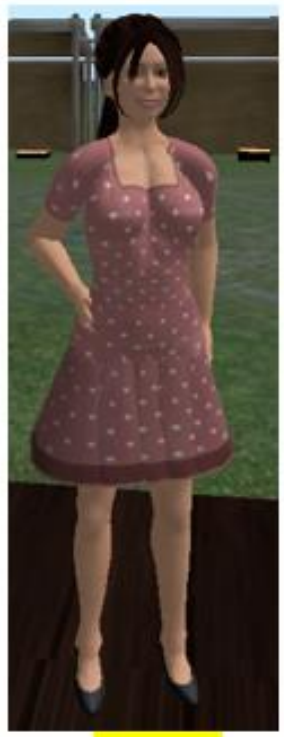

16 